\title{
STRUCTURAL DAMAGE DETECTION BY COMPARISON OF EXPERIMENTAL AND THEORETICAL MODE SHAPES
}

\author{
A Thesis \\ presented to \\ the Faculty of California Polytechnic State University, \\ San Luis Obispo
}

In Partial Fulfillment

of the Requirements for the Degree

Master of Science in Architecture with a Specialization in Architectural Engineering

by

William George Rosenblatt

March 2016 
(c) 2016

William George Rosenblatt

ALL RIGHTS RESERVED 
COMMITTEE MEMBERSHIP

TITLE: $\quad$ Structural Damage Detection by Comparison of Experimental and Theoretical Mode Shapes

AUTHOR: $\quad$ William George Rosenblatt

DATE SUBMITTED: $\quad$ March 2016

COMMITTEE CHAIR: Peter Laursen, Ph.D.

Associate Professor of Architectural Engineering

COMMITTEE MEMBER: Cole McDaniel, Ph.D.

Professor of Architectural Engineering

COMMITTEE MEMBER: $\quad$ Graham Archer, Ph.D.

Professor of Architectural Engineering 


\section{ABSTRACT}

Structural Damage Detection by Comparison of Experimental and Theoretical Mode Shapes William George Rosenblatt

Existing methods of evaluating the structural system of a building after a seismic event consist of removing architectural elements such as drywall, cladding, insulation, and fireproofing. This method is destructive and costly in terms of downtime and repairs. This research focuses on removing the guesswork by using forced vibration testing (FVT) to experimentally determine the health of a building. The experimental structure is a one-story, steel, bridge-like structure with removable braces. An engaged brace represents a nominal and undamaged condition; a dis-engaged brace represents a brace that has ruptured thus changing the stiffness of the building. By testing a variety of brace configurations, a set of experimental data is collected that represents potential damage to the building after an earthquake. Additionally, several unknown parameters of the building's substructure, lateral-force-resisting-system, and roof diaphragm are determined through FVT.

A suite of computer models with different levels of damage are then developed. A quantitative analysis procedure compares experimental results to the computer models. Models that show high levels of correlation to experimental brace configurations identify the extent of damage in the experimental structure. No testing or instrumentation of the building is necessary before an earthquake to identify if, and where, damage has occurred.

Keywords: Structural damage detection, NDT, modal analysis, Modal Assurance Criterion, MAC, Post Earthquake Assessment, Forced Vibration Testing, FVT, system identification, diaphragm stiffness, substructure stiffness, soil stiffness 


\section{ACKNOWLEDGMENTS}

l'd like to thank the Warren J. Baker and Robert D. Koob endowments that supported this research. The equipment acquired from the grant allowed this research to happen. Other students have been able to use this equipment for their research. Additionally, the same equipment has integrated into the course curriculums for undergraduate and graduate classes. 
LIST OF TABLES

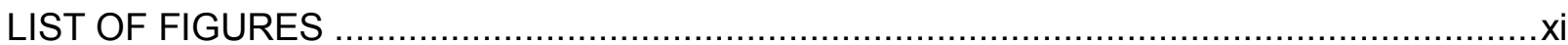

CHAPTER

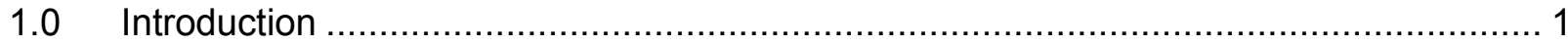

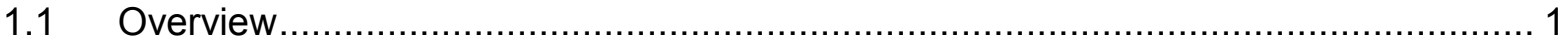

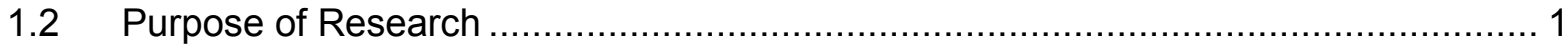

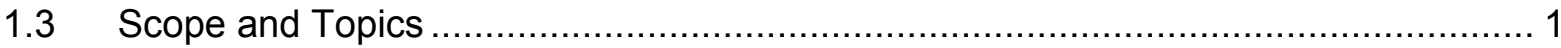

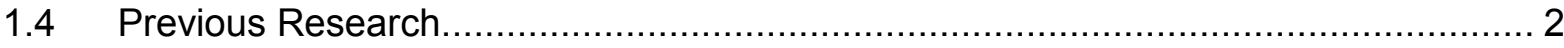

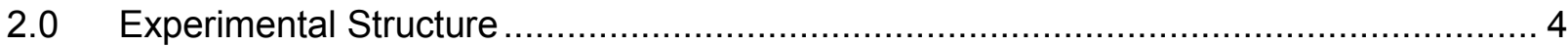

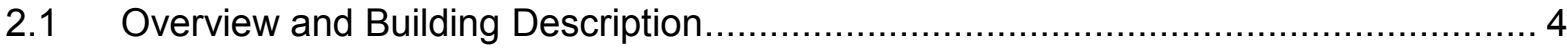

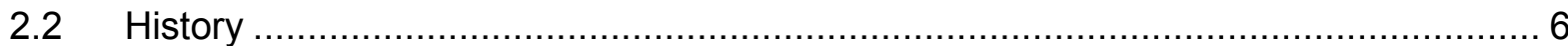

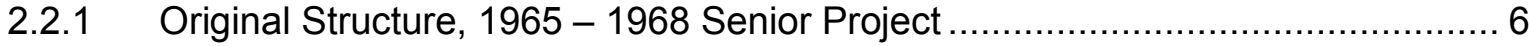

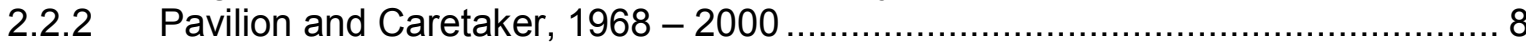

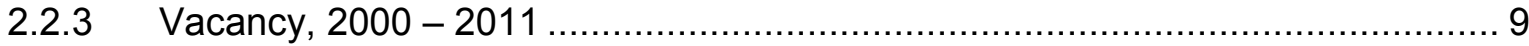

2.2.4 Structural Dynamic Field Laboratory, 2011 - Present Day ............................... 9

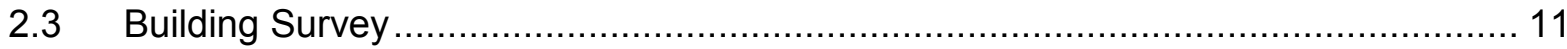

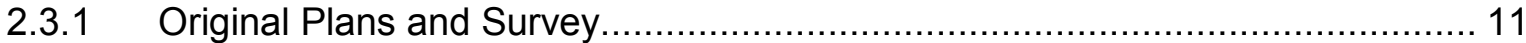

2.3.2 Exterior Survey and Structural System.................................................. 12

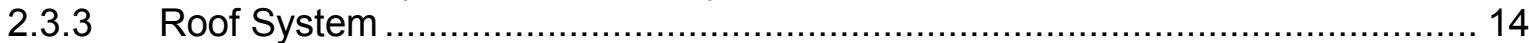

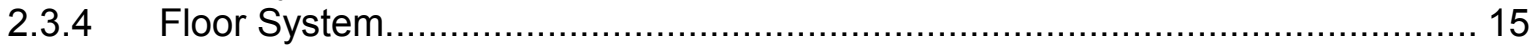

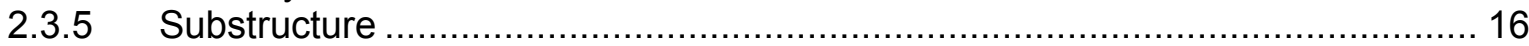

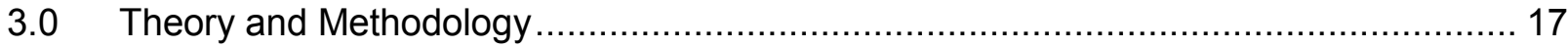

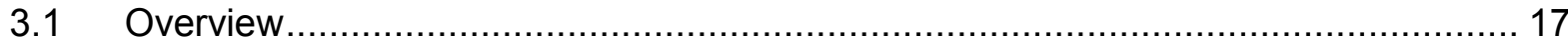

3.2 Displacement Response Amplification Factor, Rd........................................ 17

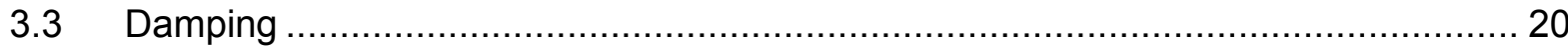

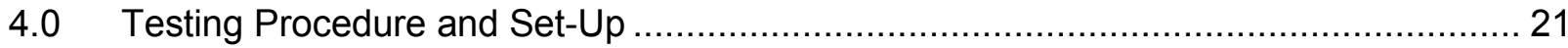

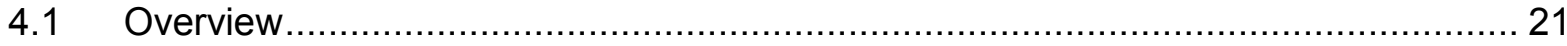

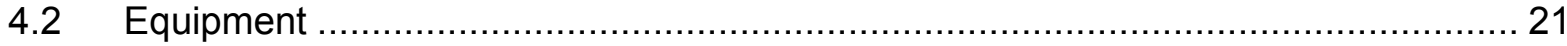

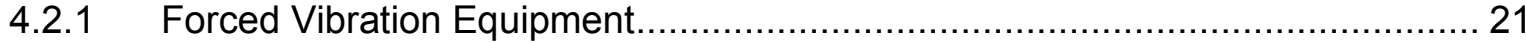

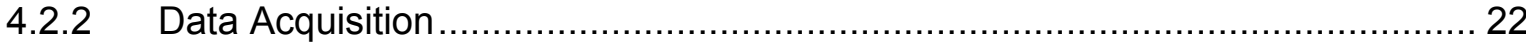

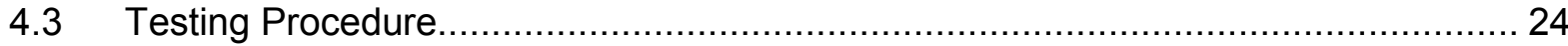

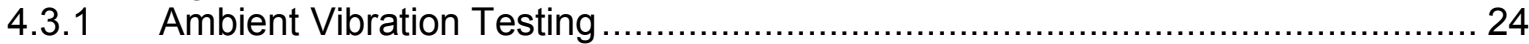

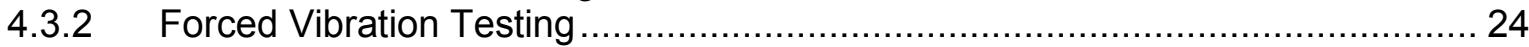

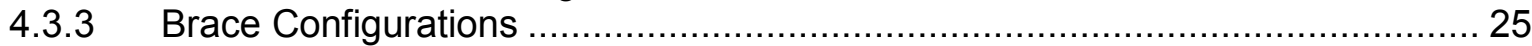




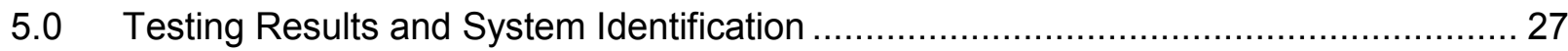

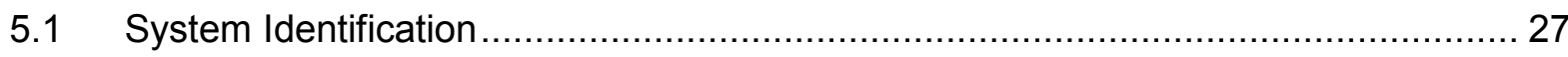

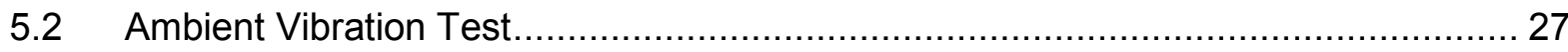

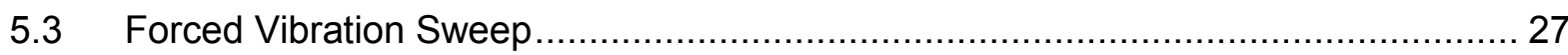

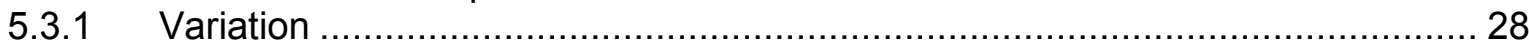

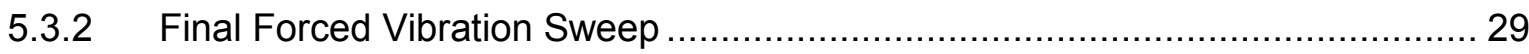

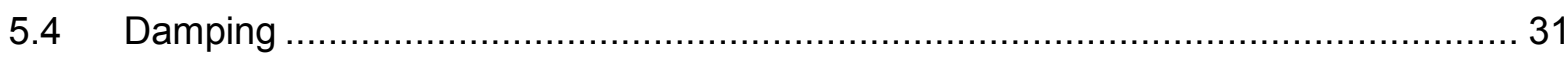

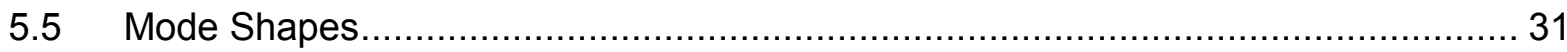

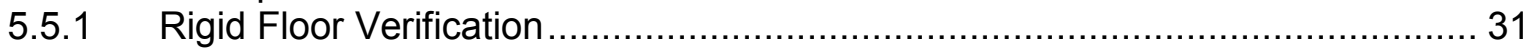

5.5.2 Raw Mode Shapes for Multiple Shaker Voltage Input .............................. 32

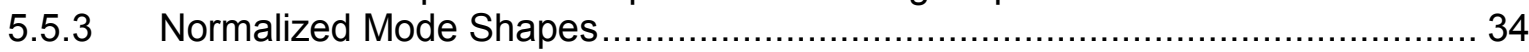

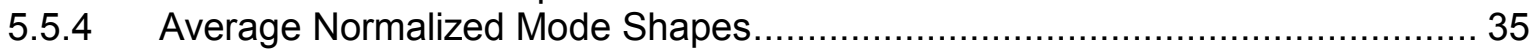

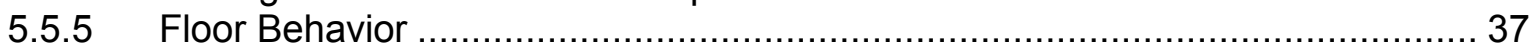

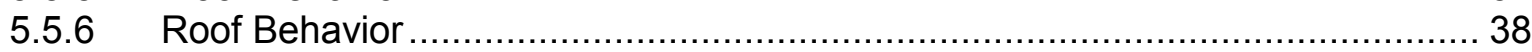

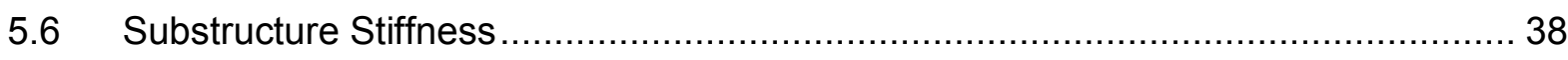

$5.7 \quad$ Lateral Force Resisting System Stiffness ................................................. 44

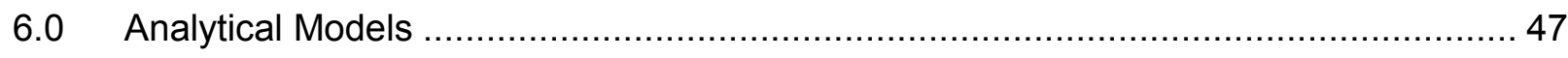

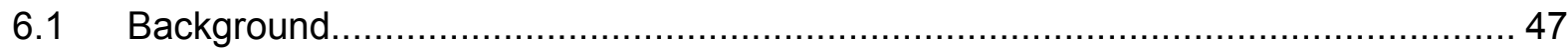

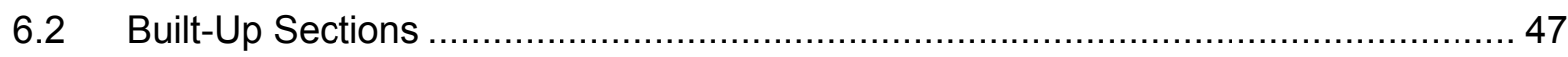

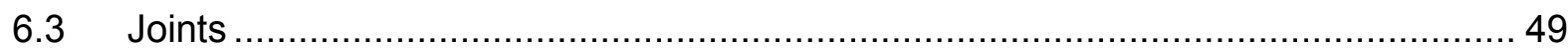

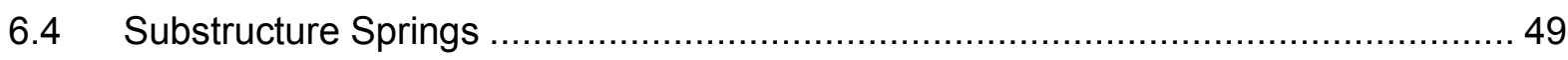

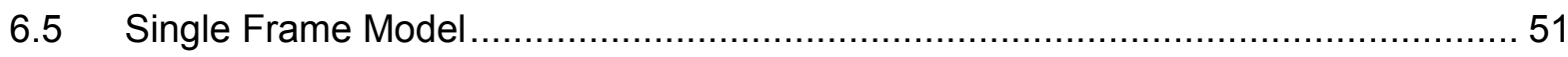

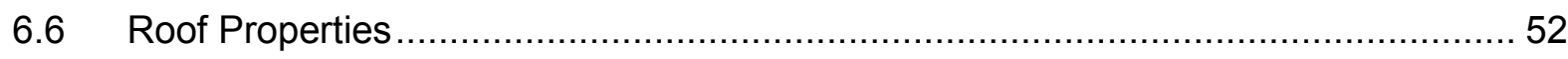

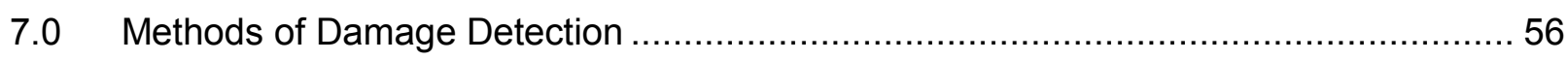

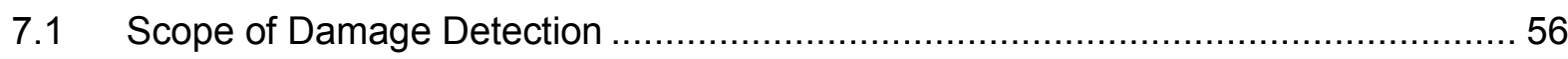

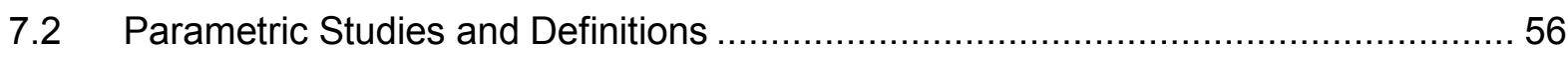

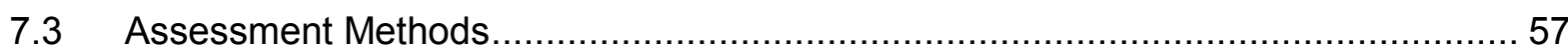

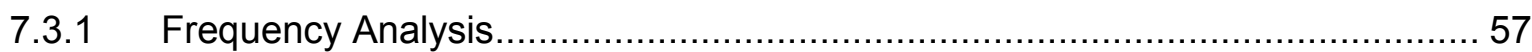

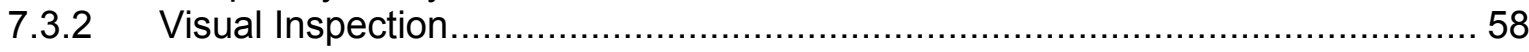

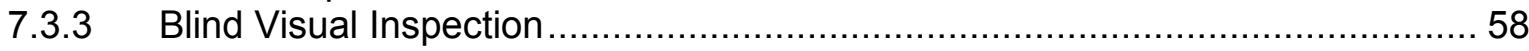

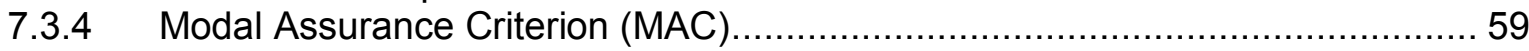

7.3.4.1 Mass Weighted Modal Assurance Criterion (MWMAC) ....................... 61

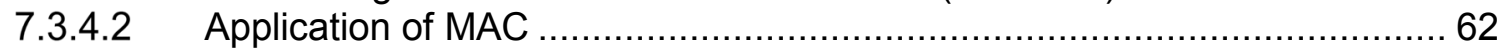

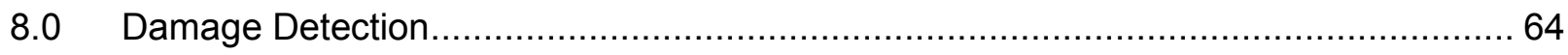

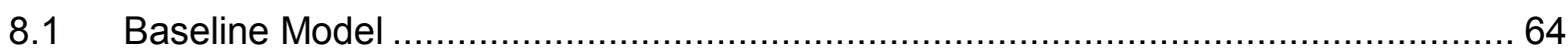

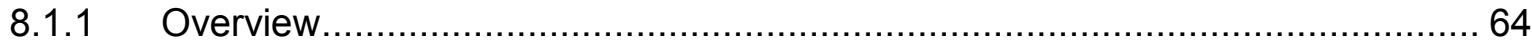

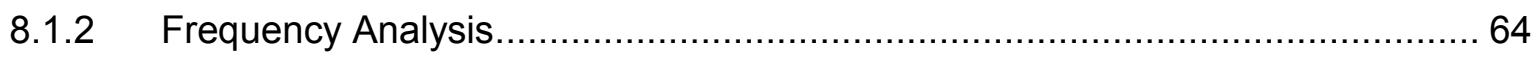

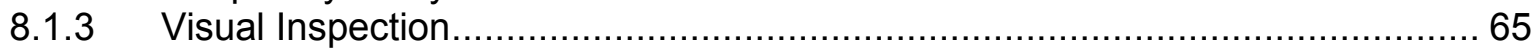

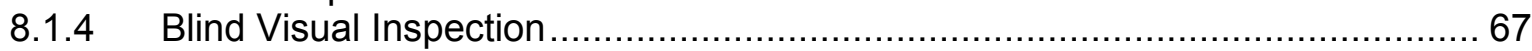

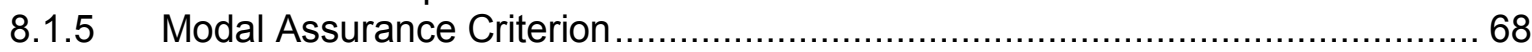




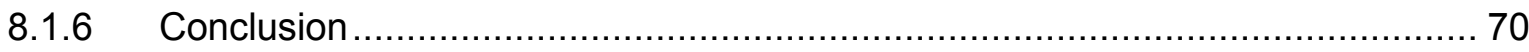

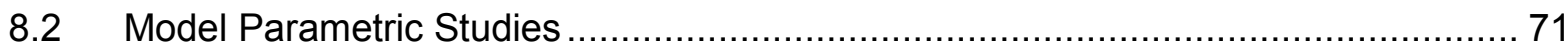

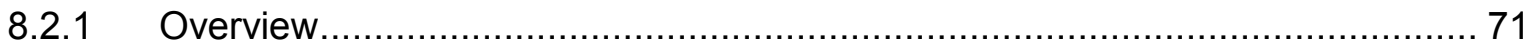

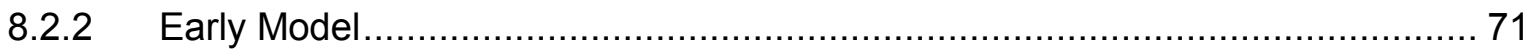

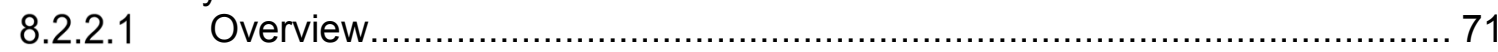

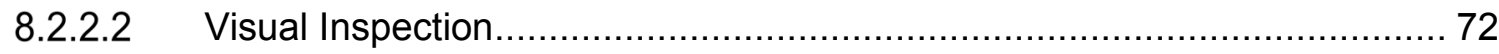

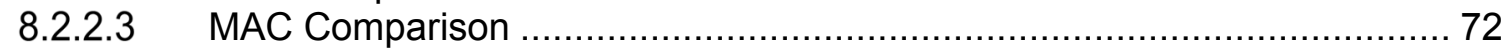

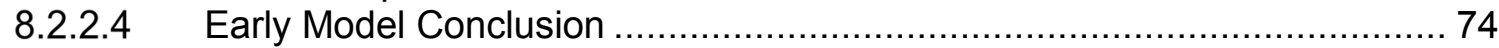

8.2.3 Substructure - Rigid .............................................................................. 74

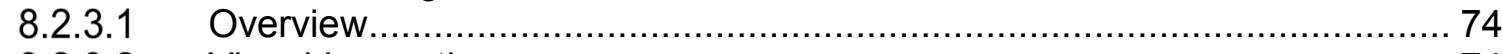

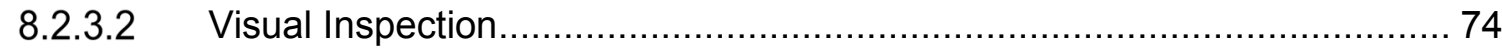

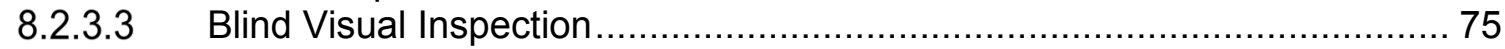

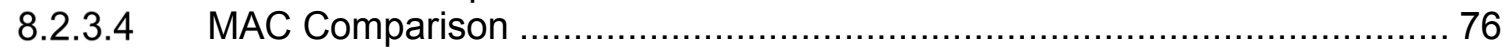

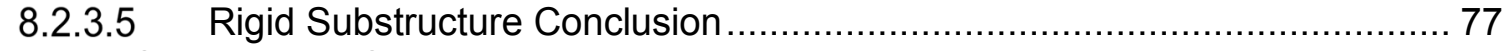

8.2.4 Substructure Springs - Variable and Individual........................................... 77

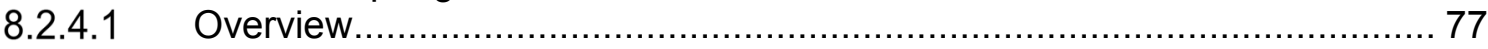

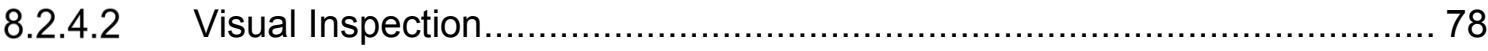

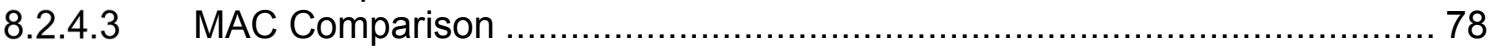

8.2.4.4 Variable Substructure Conclusion …………....................................... 79

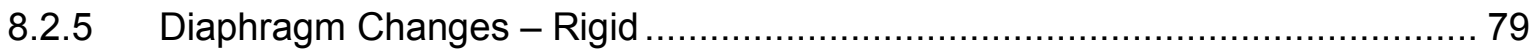

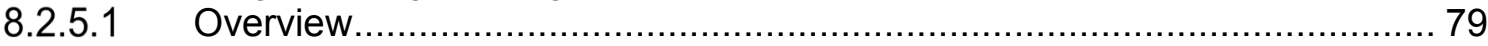

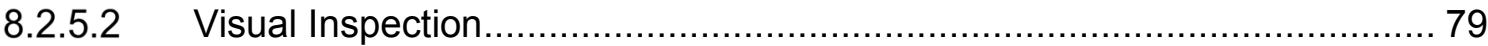

8.2.5.3 Blind Visual Comparison ............................................................ 80

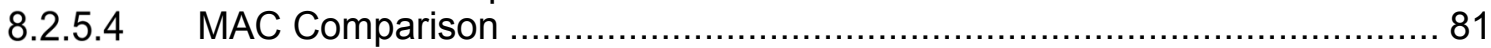

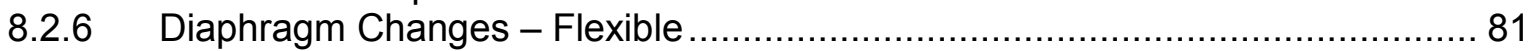

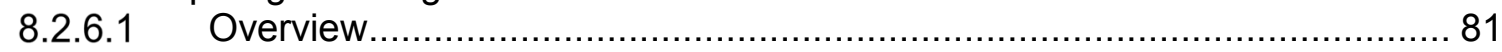

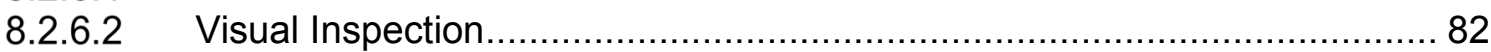

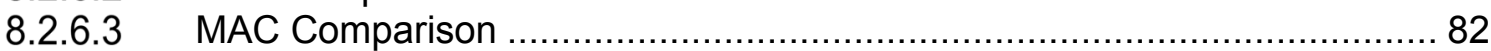

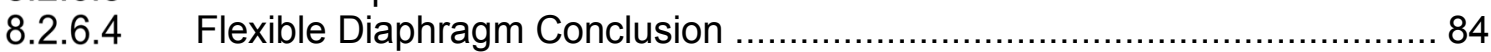

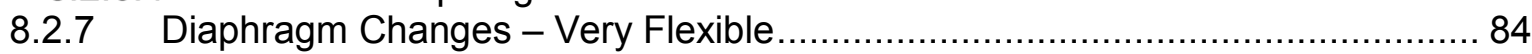

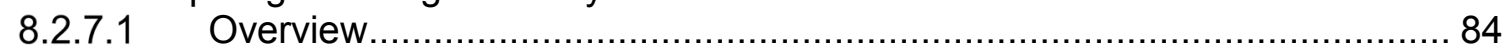

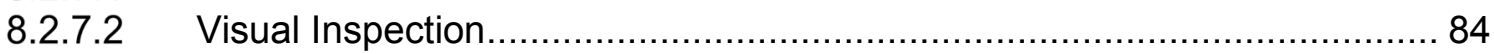

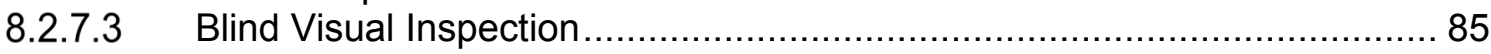

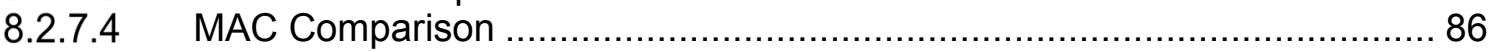

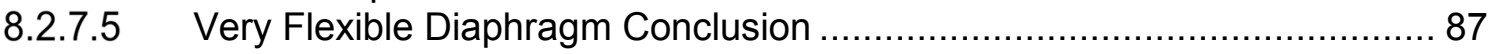

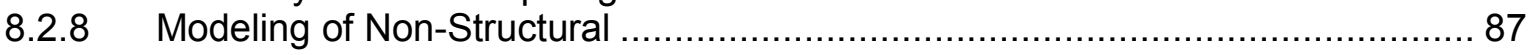

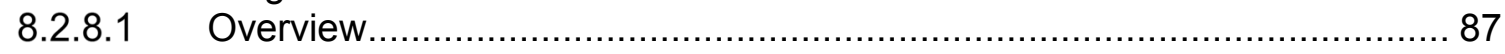

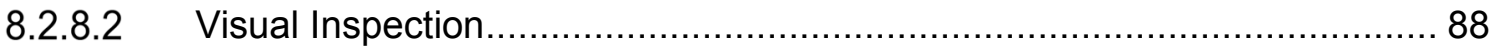

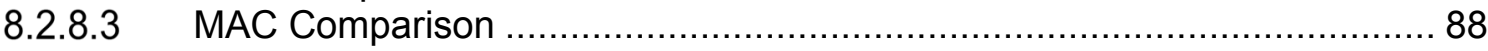

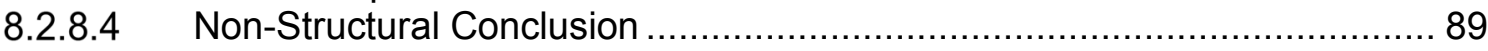

8.2.9 Conclusion to Parametric Studies ............................................................... 90

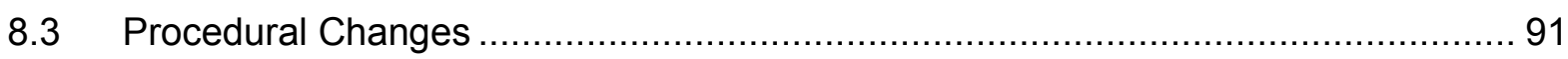

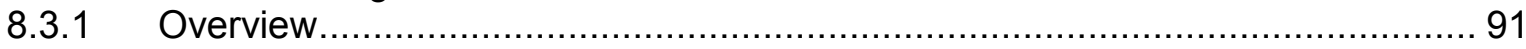

8.3.2 Nine DOF (7 Translational Roof, 1 Translational Floor, 1 Rotational Floor)....... 92

8.3.2.1 Experimental Comparison .............................................................. 92

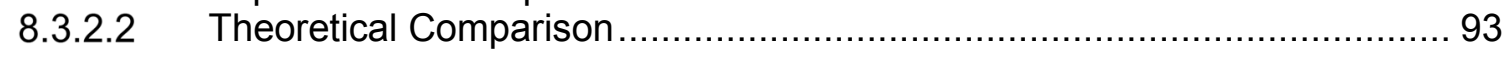

8.3.3 Five DOF (3 Translational Roof, 1 Translational Floor, 1 Rotational Floor) ....... 94

8.3.3.1 Experimental Comparison ................................................................... 94

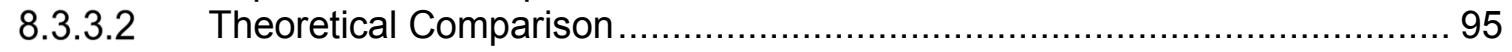

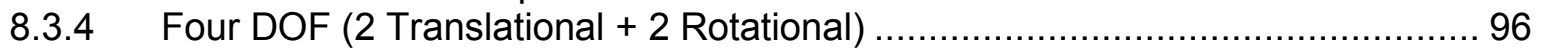




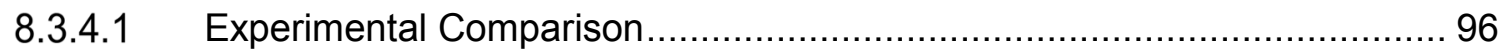

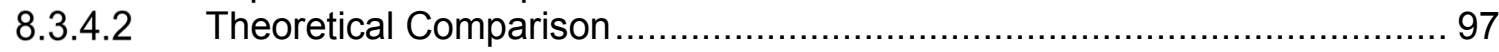

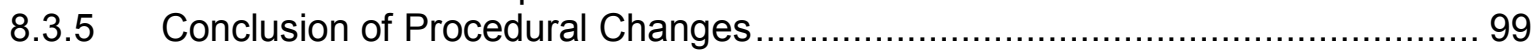

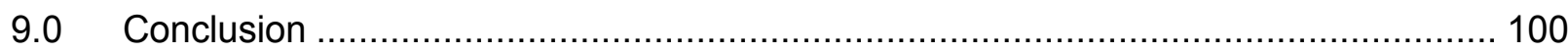

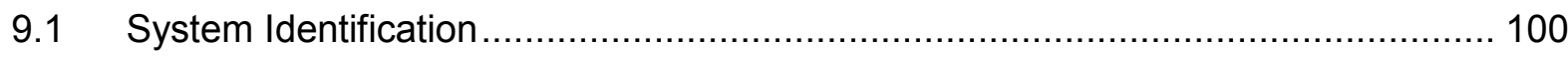

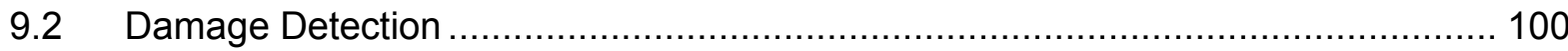

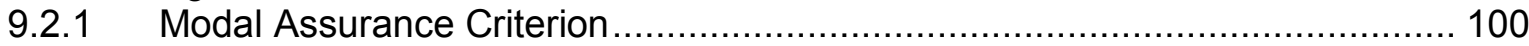

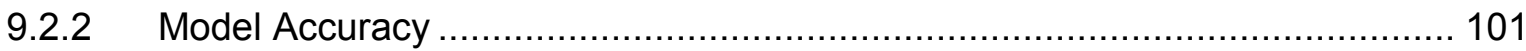

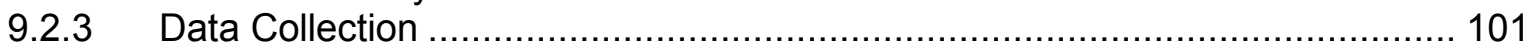

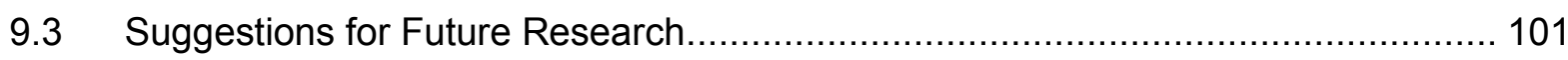

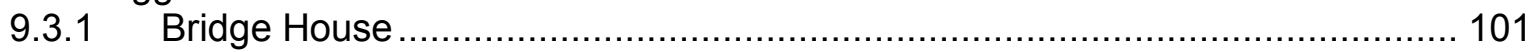

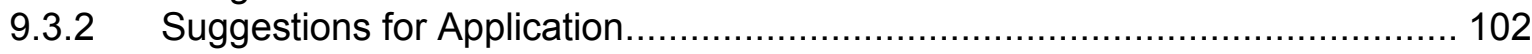

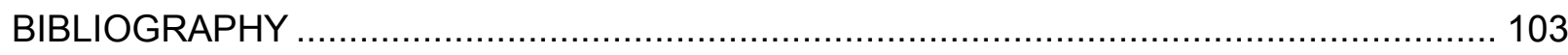

\section{APPENDICES}

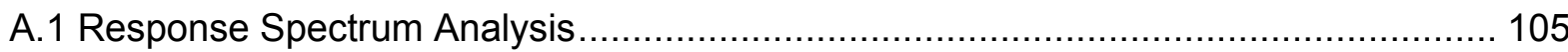

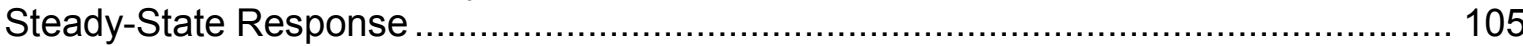

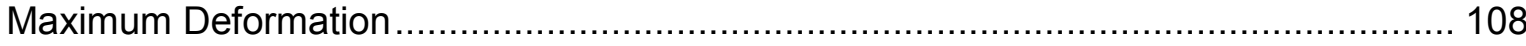

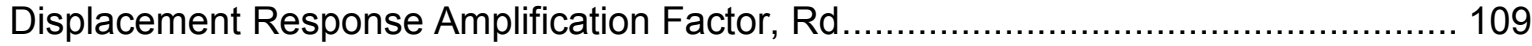

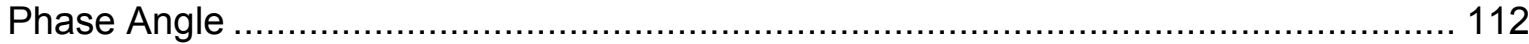

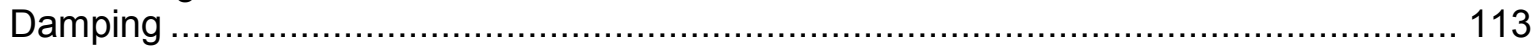

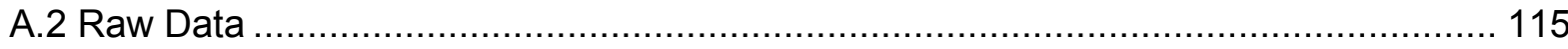




\section{LIST OF TABLES}

Table

Page

Table 5-1: Experimental frequency of first modes

30

Table 5-2: Tested damping coefficients.....................................................................

Table 5-3: LFRS stiffness for All Braces On ..................................................................

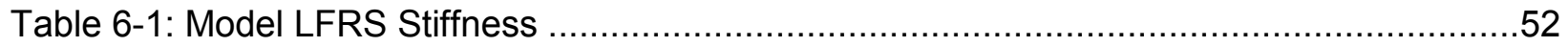

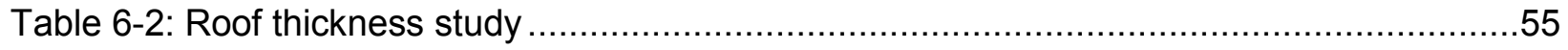

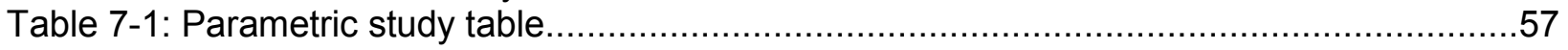

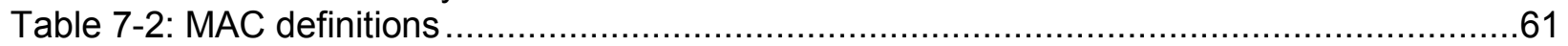

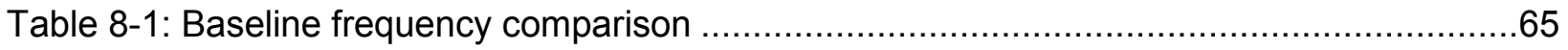

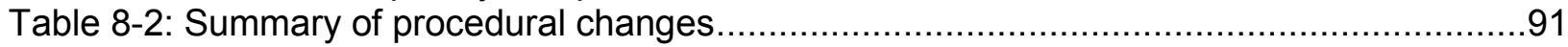




\section{LIST OF FIGURES}

Figure $\quad$ Page

Figure 2-1: Bridge House map (left), Bridge House exterior (right) ............................... 4

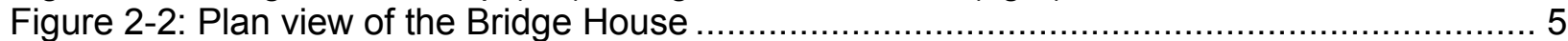

Figure 2-3: South elevation of the Bridge House ............................................................. 5

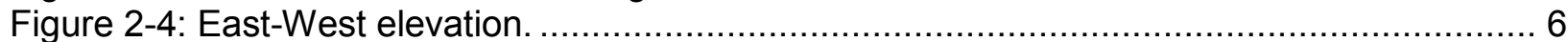

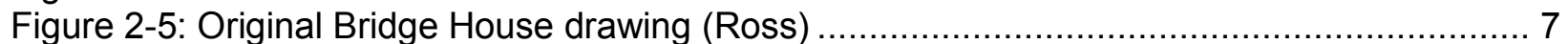

Figure 2-6: Bridge House completed (Ross) ............................................................ 8

Figure 2-7: Bridge House in the 1980's (Ross) ........................................................... 8

Figure 2-8: Bridge House circa 2011 (Planas, 2011) ................................................. 9

Figure 2-9: Bridge House in current state ................................................................. 11

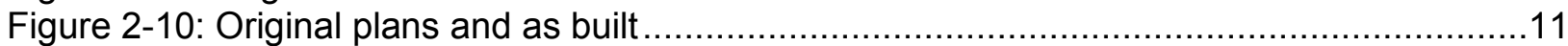

Figure 2-11: Built up column (left), column detail (right) ........................................12

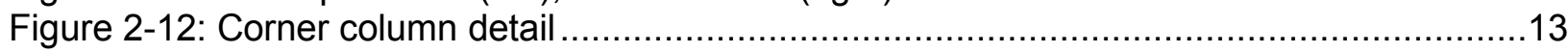

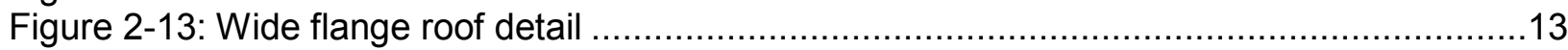

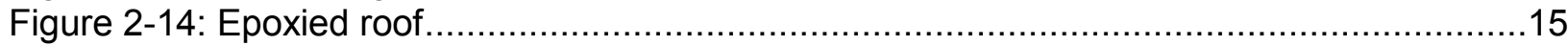

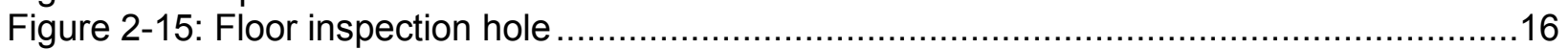

Figure 2-16: Examples of the various piers at the Bridge House ......................................16

Figure 3-1: Dynamic Response Displacement Amplification..............................................19

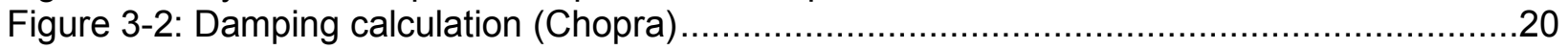

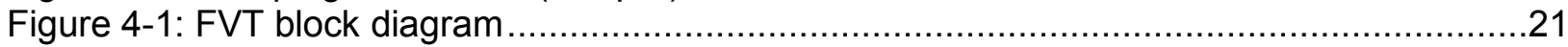

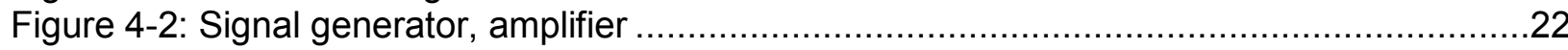

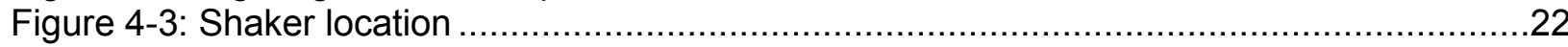

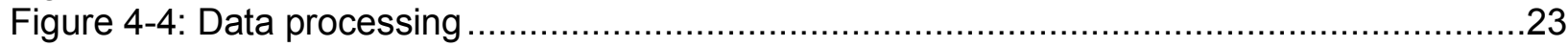

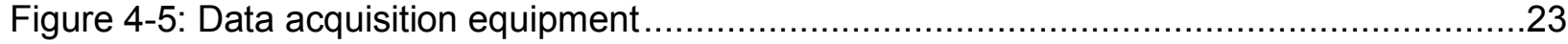

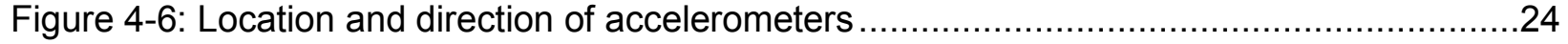

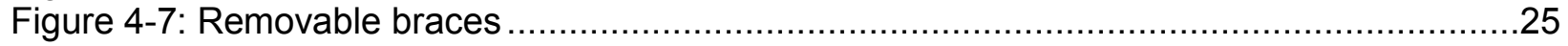

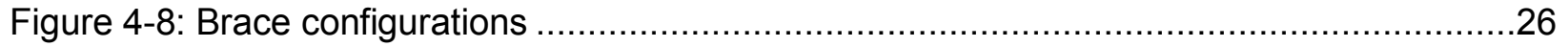

Figure 5-1: Location and direction of accelerometer used for FVT sweep .........................27

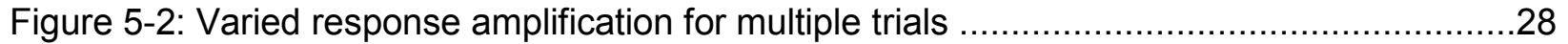

Figure 5-3: Final experimental response forced vibration sweep .....................................30

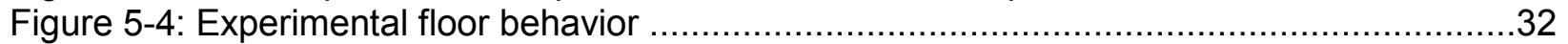

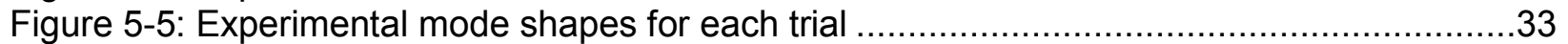

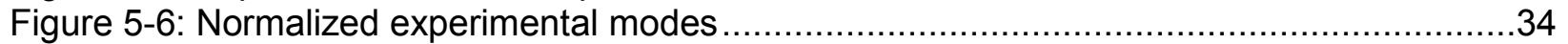

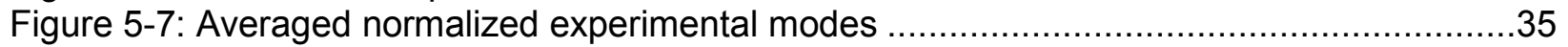

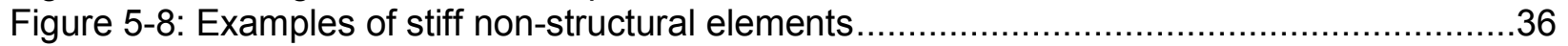

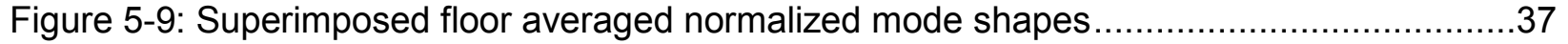

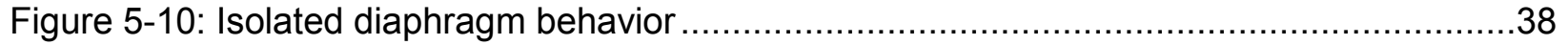

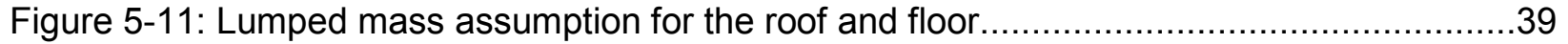

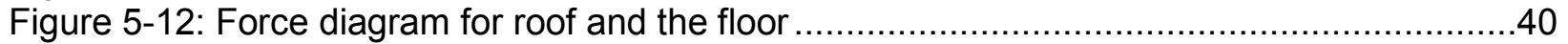

Figure 5-13: Suspected as built (left), Theoretical model (right) .....................................42

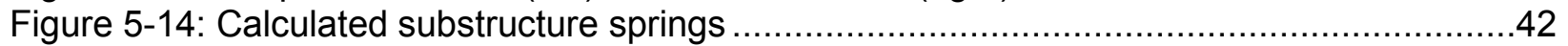

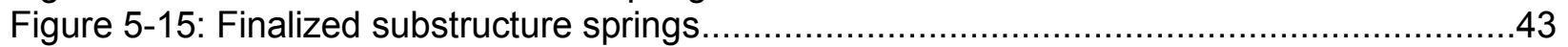

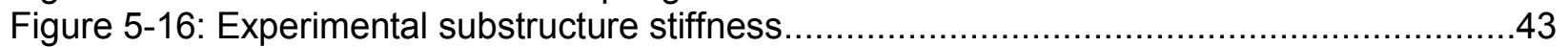

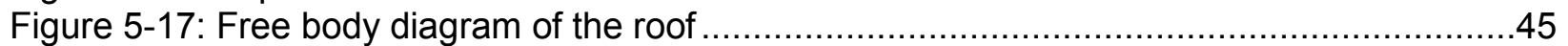

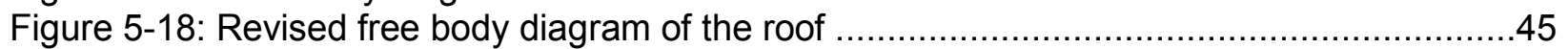

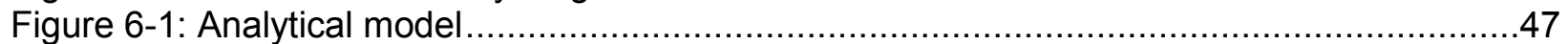

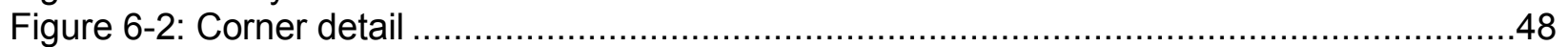

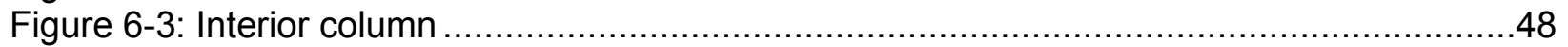

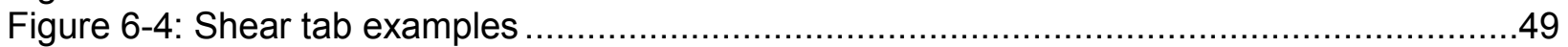


Figure 6-5: Averaged experimental substructure stiffness .............................................50

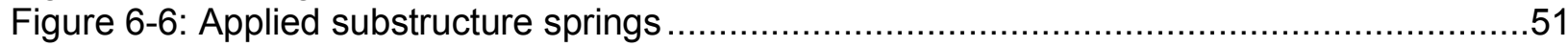

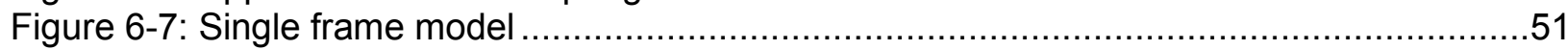

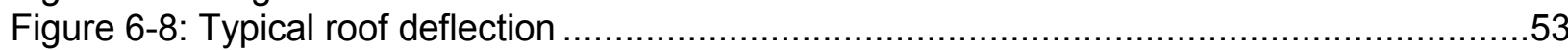

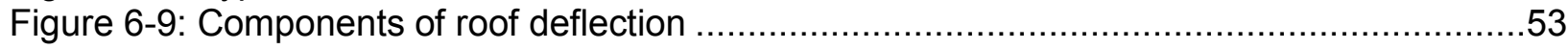

Figure 6-10: Distributed load assumption for roof stiffness identification .............................54

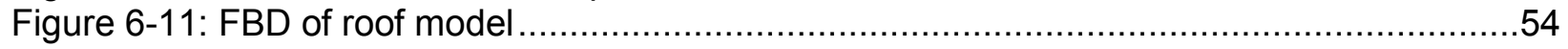

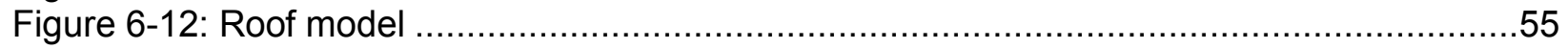

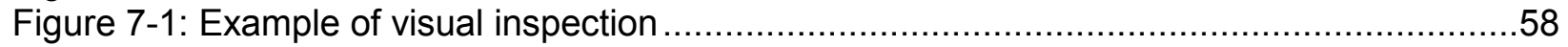

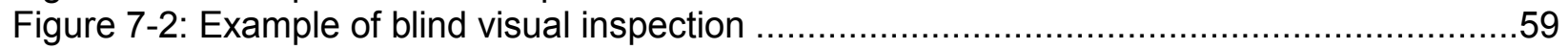

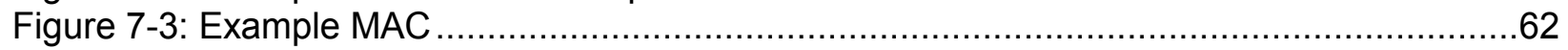

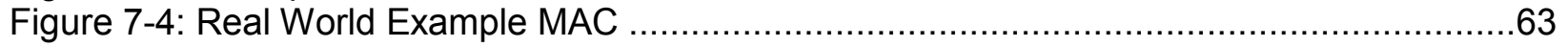

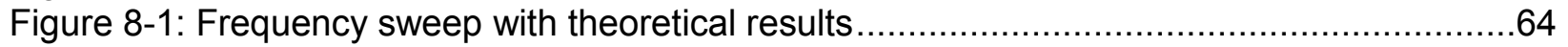

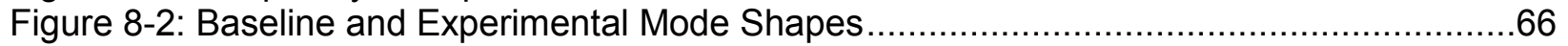

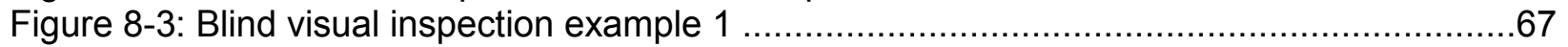

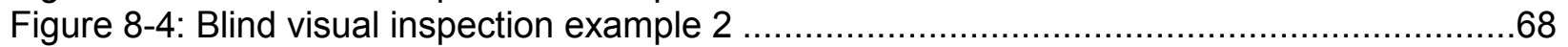

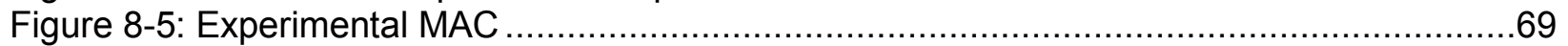

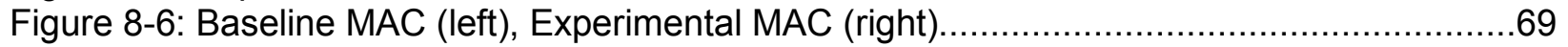

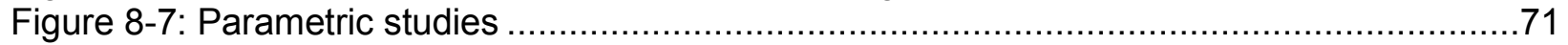

Figure 8-8: Early model mode shapes ......................................................................

Figure 8-9: Early Theoretical MAC (left), Baseline MAC (right) ......................................73

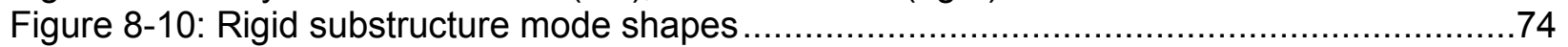

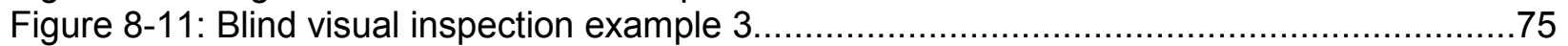

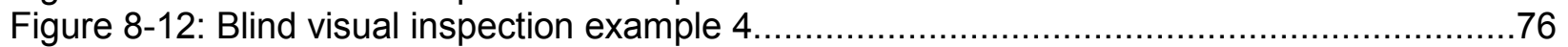

Figure 8-13: Rigid Substructure Theoretical MAC (left), Baseline MAC (right)......................77

Figure 8-14: Variable substructure mode shapes ........................................................78

Figure 8-15: Variable Substructure MAC (left), Baseline MAC (right) ...............................78

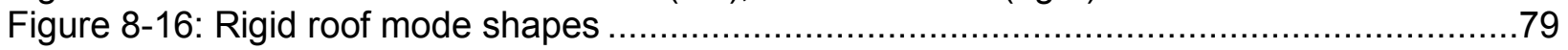

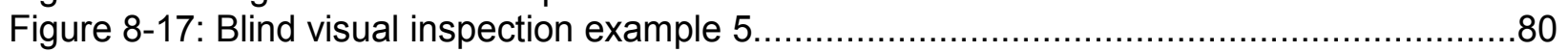

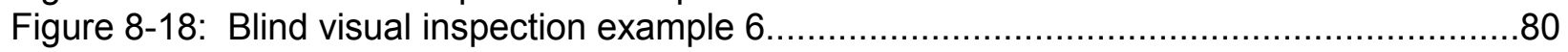

Figure 8-19: Rigid Roof Theoretical MAC (left), Baseline MAC (right) ............................. 81

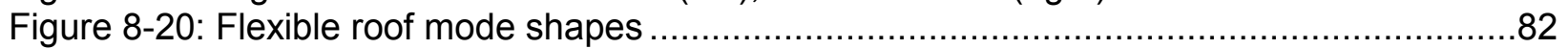

Figure 8-21: Flexible Roof Theoretical MAC (left), Baseline MAC (right) .............................83

Figure 8-22: Flexible Roof Theoretical MAC (left), Experimental MAC (right) ........................83

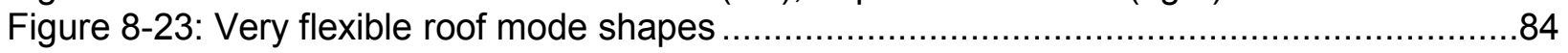

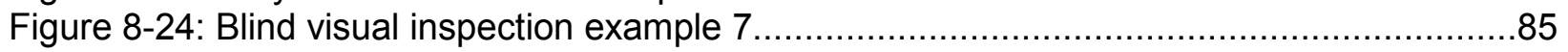

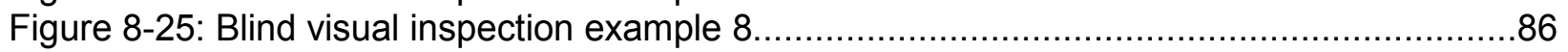

Figure 8-26: Very Flexible Roof Theoretical MAC (left), Baseline MAC (right).......................86

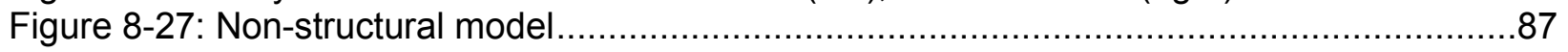

Figure 8-28: Increased non-structural mode shapes ............................................... 88

Figure 8-29: Non-Structural Theoretical MAC (left), Baseline MAC (right) ...........................89

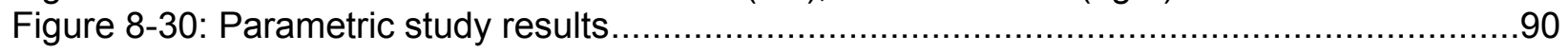

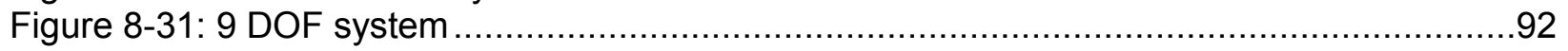

Figure 8-32: Experimental MAC - 9 DOF (left), Experimental MAC (right) - 14 DOF .............93

Figure 8-33: Baseline MAC - 9 DOF (left), Baseline MAC (right) - 14 DOF ..........................93

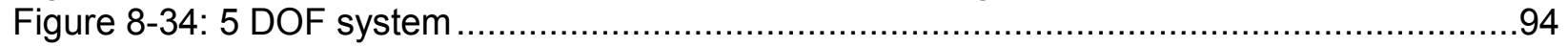

Figure 8-35: Experimental MAC - 5 DOF (left), Experimental MAC (right) - 14 DOF .............94

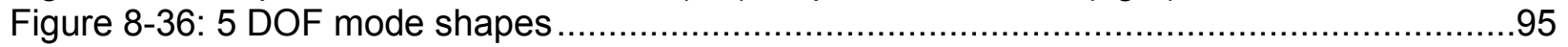

Figure 8-37: Baseline MAC - 5 DOF (left), Baseline MAC (right) - 14 DOF ........................96

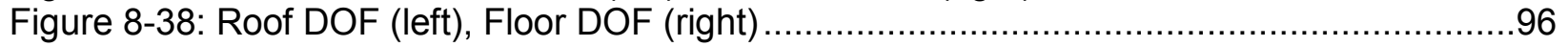

Figure 8-39: Experimental MAC - 4 DOF (left), Experimental MAC (right) - 14 DOF ..............97 


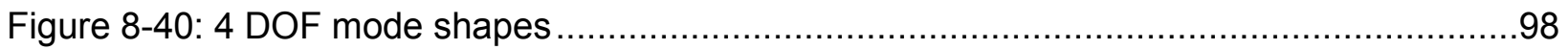

Figure 8-41: Baseline MAC - 4 DOF (left), Baseline MAC (right) - 14 DOF .........................98

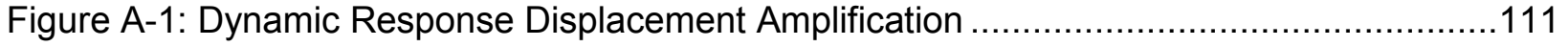

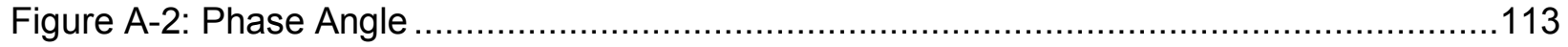

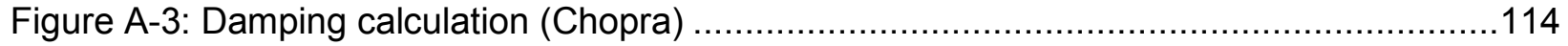




\subsection{INTRODUCTION}

\subsection{Overview}

The objective of this thesis is to use temporary instrumentation and system identification through the use of forced vibration testing to identify the location of structural damage in a building after a seismic, wind, or blast event. The proposed methods in this thesis need no previous identification or knowledge of the building to identify if, and where, structural damage has occurred.

\subsection{Purpose of Research}

Current investigative techniques after a lateral force event consist of destructive testing. Drywall, ceiling tiles, fireproofing, insulation, and other like elements are required to be removed in order to inspect steel members and connections. These methods are intrusive, they displace tenants, and are costly, both in terms of repairs and lost revenues, especially in large buildings.

The methods used in this research will mitigate the need for guesswork when assessing the structural robustness of a building. They has the potential to give the engineer an " $\mathrm{x}$-ray" look into the building to determine if, and where, further inspections are necessary. If successful, the damaged detection system could expedite and minimize the destructive inspections needed. This would lower costs for building owners and tenants by shortening the turnaround time for the building to return to service.

\subsection{Scope and Topics}

The main focus of this research is system identification through forced vibration testing and damage detection through comparison of experimental and theoretical mode shapes. To help establish the results of this thesis, it will be broken down as linearly as possible. Much work is needed to be done in order implement this system into a turn-key solution; it is the hope 
of the author that this thesis will guide and help future students who conduct forced vibration testing.

First, this thesis will begin with a discussion of the test structure. The history of the structure is unique, along with the as-built conditions. Next, a discussion of forced vibration testing (FVT), dynamic response, and system identification will be presented. Next, the practices of the test set-up and procedure will outline the data acquisition. The system identification methods used for FVT are unique to this research and will be outlined for both theory and practice. These results will be implemented into a finite element computer model.

Focus will be shifted on blind structural damage detection, or the ability to predict damage, with no previous information about the structure. A series of parametric studies will be conducted. The first set of studies will change parameters in the model. These parametric studies will focus on varying assumptions made during modeling and.

Second, a series of studies will examine how many degrees-of-freedom (DOF) are needed to test a structure. Simultaneous data acquisition of many accelerometers is costly; data acquisition using "roaming" accelerometers is time consuming and has data processing difficulties. Ideally, there is a balance in terms of cost versus time.

The results of these studies, along with observations made during testing, will be summarized in the conclusion. Suggestions for further research, along with suggestions for application in a real world scenario, will be discussed. The robustness of the system identification will be addressed.

\subsection{Previous Research}

The majority of the research done on in this thesis will build upon an accumulation of research conducted by former graduate students and faculty-led research at Cal Poly, San Luis 
Obispo. Much of this research was conducted by professors Graham Archer, Ph.D. and Cole McDaniel, Ph.D. (McDaniel, 2012). Archer and McDaniel have successfully used ultralow-force, linear vibrating shakers to perform system identification on multiple low rise buildings. Archer and McDaniel have refined testing results to a turn-key system for the majority of building types. A paper titled Influence of Boundary Conditions on Building Behavior (Raney et. al., 2015) successfully calculated the stiffness of boundary conditions through both forced vibration tests and static loading tests. This research was used to show that system identification methods can be expanded to calculate previously unknown parameters of a structure.

While many studies impacted the direction of this research, a few major studies were used to help converge the ideas for this thesis. The first was a thesis called System Identification Of A Bridge-Type Building Structure, (Ramos, 2013). Ramos performed dynamic studies on the same structure used for this thesis. In addition, Ramos' work outlined and discovered many of the unique challenges particular to the test structure. Archer and Graham expanded this research to have limited success using damage detection (Archer, 2014). Additionally, the thesis titled Structural Damage Detection Utilizing Experimental Mode Shapes (Gerbo, 2014) expanded on the idea that damage could precisely be identified by comparing experimental mode shapes to theoretical mode shapes. Gerbo tested a two-story laboratory structure with removable braces to simulate damage. Gerbo compared the experimental results to a suite of finite element models to successfully detect the different brace configurations. 


\subsection{EXPERIMENTAL STRUCTURE}

The name of the structure used for the testing in this thesis is called the Bridge House.

The following section details the history, overview, and structural evaluation of the Bridge House.

\subsection{Overview and Building Description}

The Bridge House is located approximately two miles from the center of Cal Poly's campus in the Experimental Structures Facility. The Experimental Structures Facility is an allocated region of Cal Poly's campus where students can design and construct full scale structures. The Experimental Structures Facility is located in a geographical region known as Poly Canyon, which is named for its rolling terrain. Many of the structures in the Experimental Structures Facility are designed to interact with the hilly and undulating terrain.
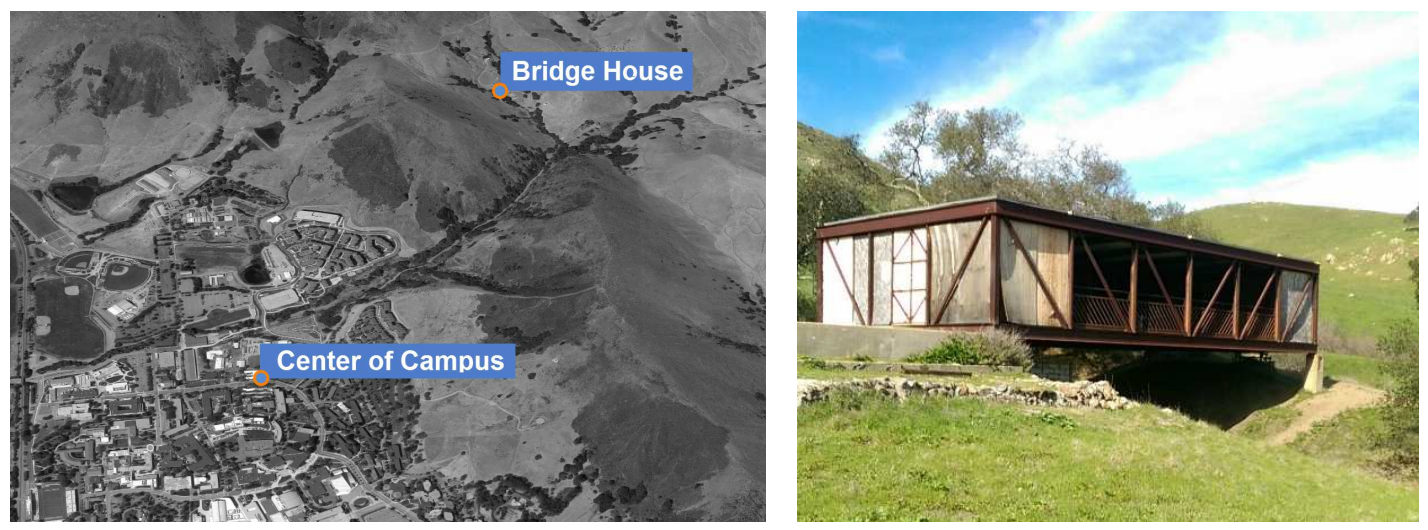

Figure 2-1: Bridge House map (left), Bridge House exterior (right) 


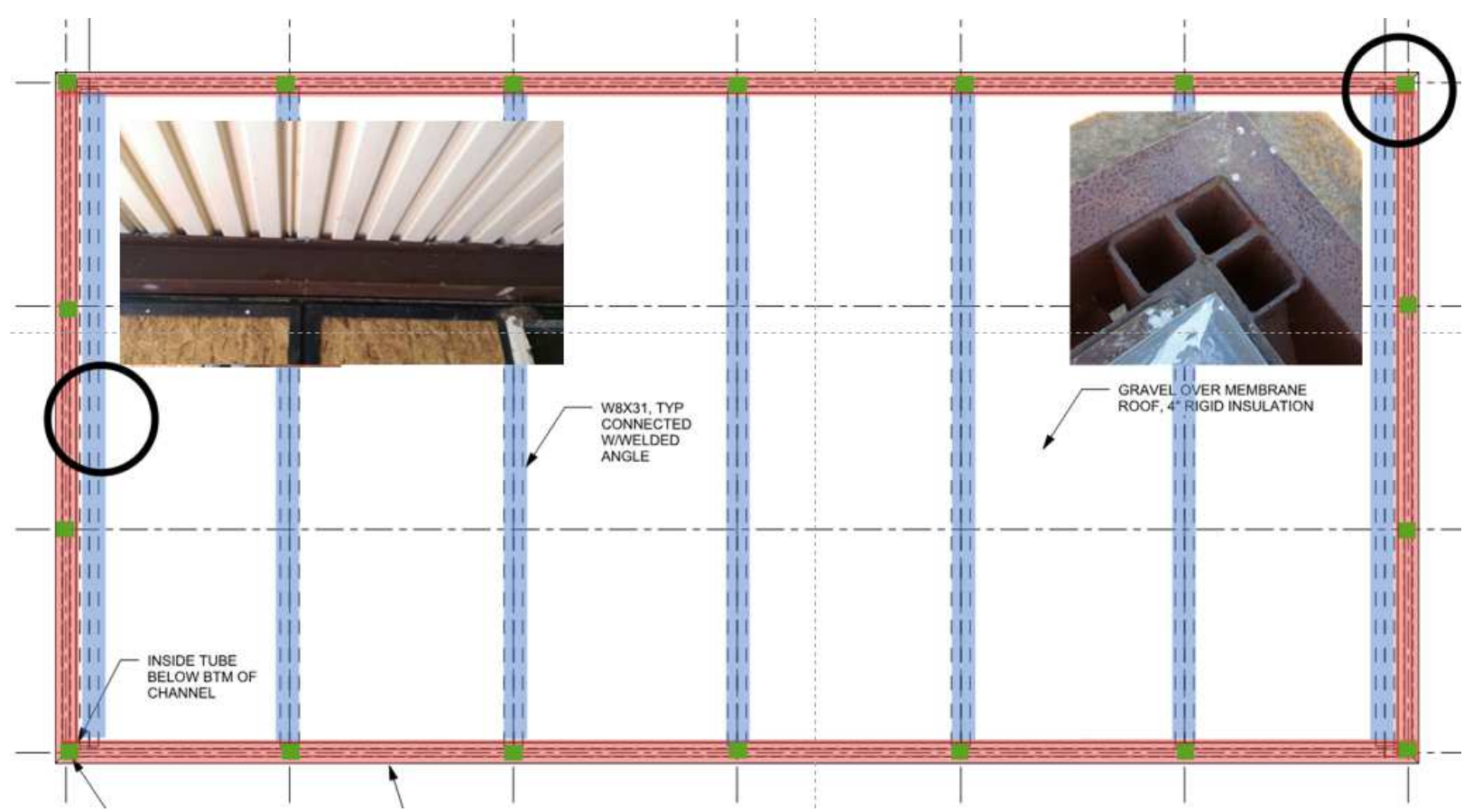

Figure 2-2: Plan view of the Bridge House

The Bridge House was never designed for lateral loads, although the lateral force resisting system can be classified as follows. The lateral force resisting system in the EastWest direction is composed of a truss with welded HSS $3 \times 3 \times 1 / 4$ diagonals.

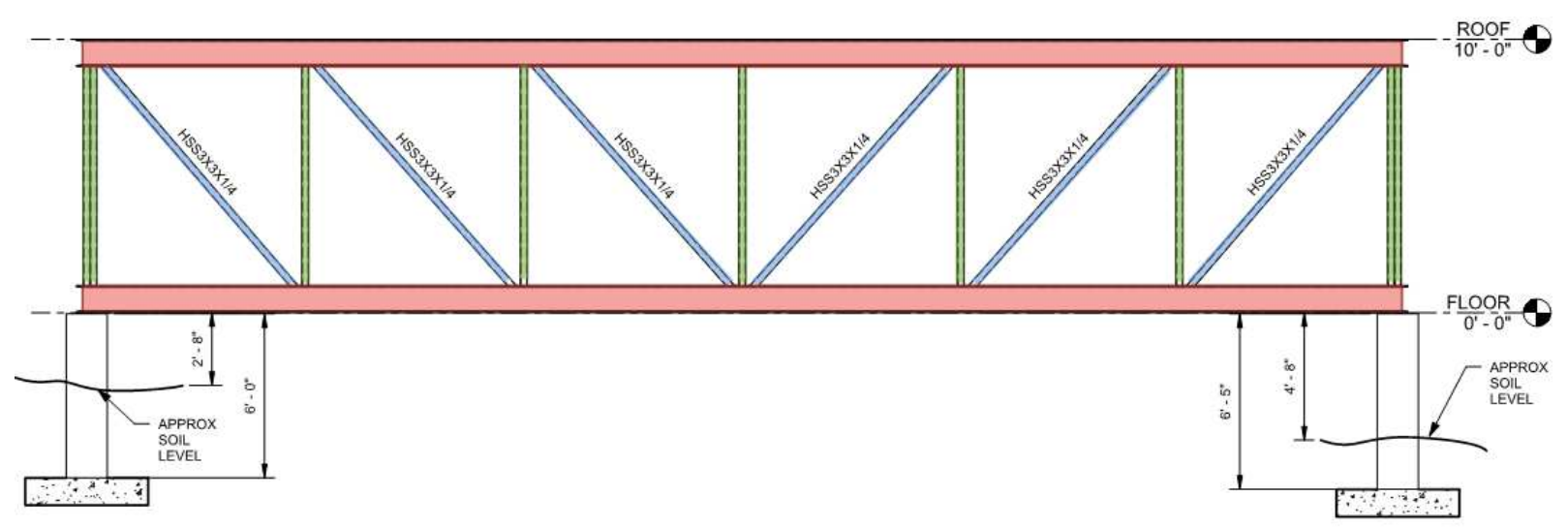

Figure 2-3: South elevation of the Bridge House

In the North-South direction, the lateral force resisting system is composed of a hybrid Vierendeel truss and brace frame system. The braces in the North-South system are HSSS $3 \times 2 \times 1 / 4$ are removable to facilitate dynamic testing. 


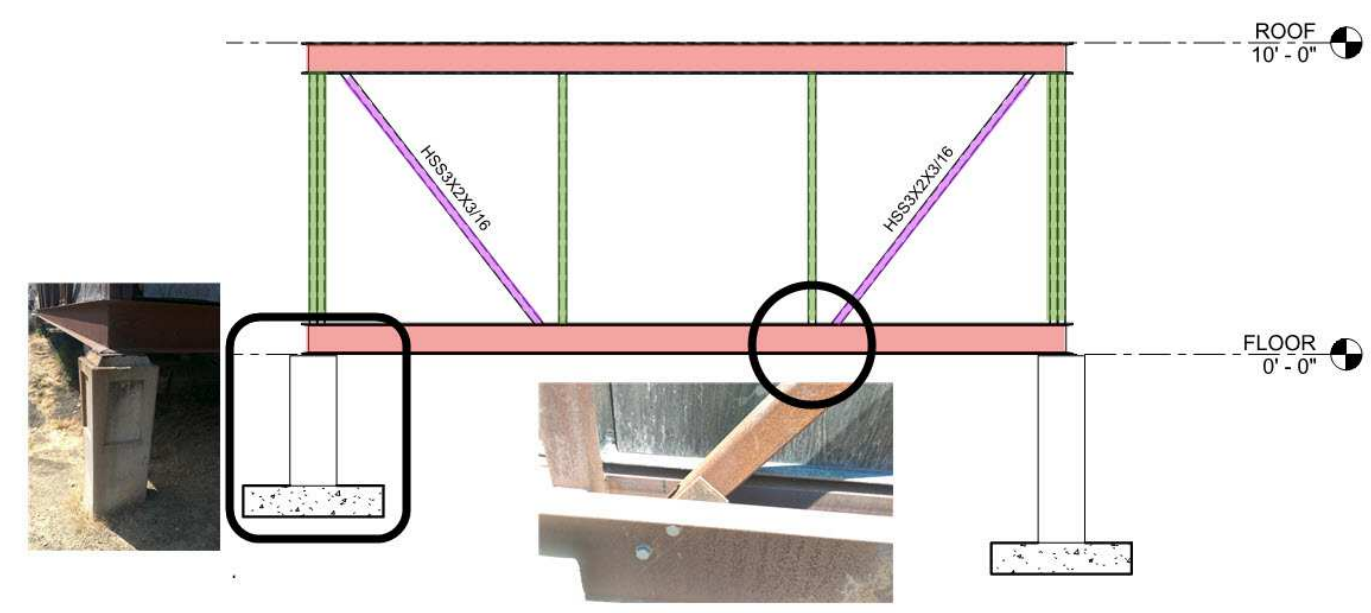

Figure 2-4: East-West elevation.

The Bridge House sits on four concrete pedestals. Each pedestal is approximately the same height at the top of concrete, although, the total height of each pedestal, as well as it's embedment into the soil, varies.

\subsection{History}

\subsubsection{Original Structure, 1965 - 1968 Senior Project}

The origins of the Bridge House are a conglomerate of several inspirations that converged together. In 1965, several students were planning a senior project in which they wanted to showcase California's rolling landscape. At this time, the Experimental Structures Facility was in its infancy and the students chose the location for their senior project. Because of the location, the students decided to create a house similar to a bridge that showcased the terrain. At this time, the college was looking to incorporate lodging arrangements for temporary lectures, professors, and visitors. The Bridge House was the obvious choice to showcase Cal Poly's unique terrain and surroundings. 


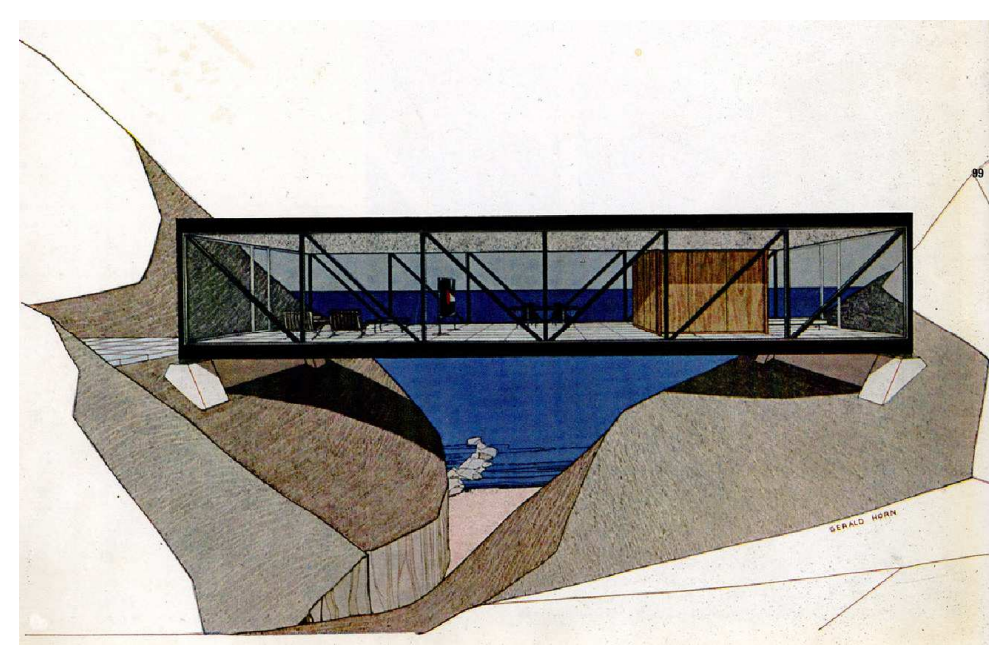

Figure 2-5: Original Bridge House drawing (Ross)

Concurrently during 1965, Kaiser Steel approached California Polytechnic University, San Luis Obispo with a proposal to sponsor a student-built project that showcased a line of weathered steel. Kaiser wanted to showcase their new line of steel in an interesting way as well as test how the steel would perform in a corrosive, semi-marine environment. At this point, Kaiser was paired up with the senior project team, and Kaiser approved the project. In 1966, Kaiser contacted the senior project design team letting them know that Kaiser's contribution to the project was on hold due to the manufacturing needs of the Vietnam War. In 1967, a group of students got together and resurrected the project. Kaiser agreed to uphold their donation, and the Bridge House was completed in 1968. That same year, the Bridge House won the AISC Architectural Award for Excellence (Garlow, 2016). 

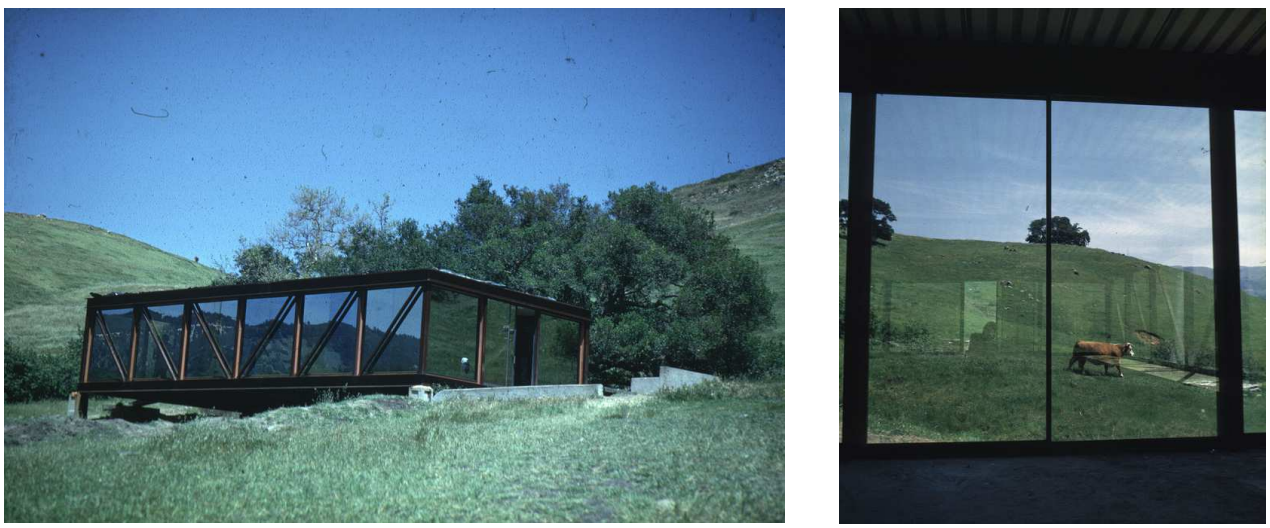

Figure 2-6: Bridge House completed (Ross)

\subsubsection{Pavilion and Caretaker, 1968 - 2000}

Original plans for the Bridge House were to use the space as lodging for visiting lecturers and faculty to the campus. For the first few decades, it served as a pavilion, classroom, and temporary lodging for visiting guests. During the 1970 s and 1980 s, Poly Canyon grew and several structures infilled the canyon. Student caretaker housing units were built and infrastructure was installed. During the late 1980s, the Bridge House was turned into a student caretaker residence. Some of the glass panels on the backside of the Bridge House were removed and replaced with timber and cold formed walls in order to increase the privacy for resident (Planas, 2011).
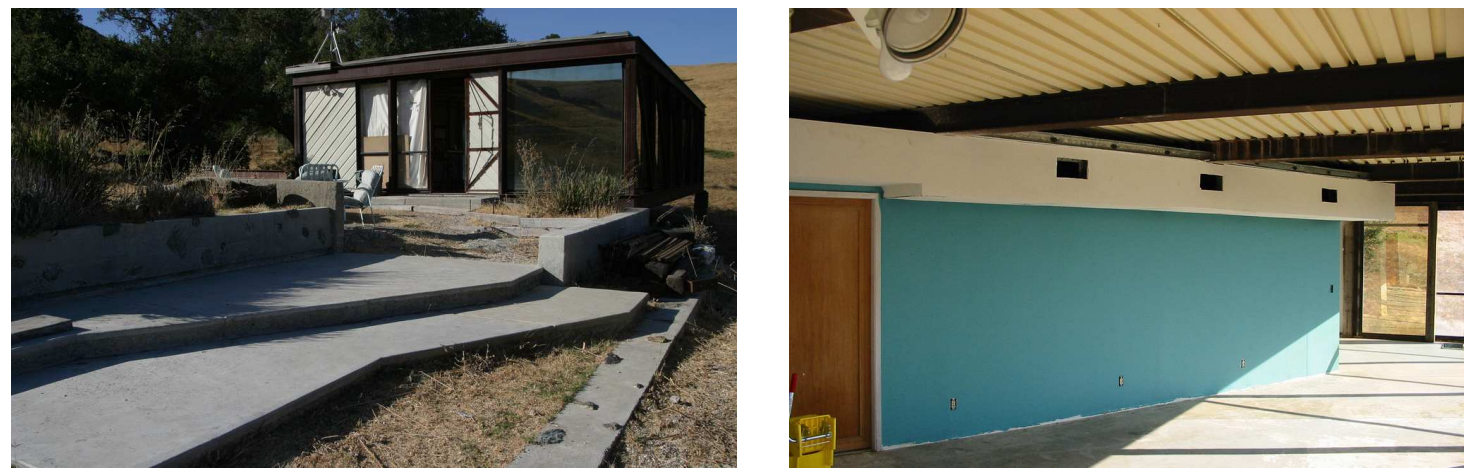

Figure 2-7: Bridge House in the 1980's (Ross) 


\subsubsection{Vacancy, $2000-2011$}

In 2000, a group of Architectural Engineering students created a senior project to install a water ponding system on the roof of the Bridge House. The students mistakenly calculated the seismic loads and deemed the structure was unsafe. The building was condemned and the student caretaker living in the residence was evicted while further investigation was done. A licensed structural engineer was hired to assess the structure. He discovered the students' mistake and approved the structure for occupancy. However, this additional attention resulted in additional scrutiny and attention from the university and the Bridge House was never approved for occupancy. Several other habitable structures in Poly Canyon suffered similar fates and were condemned. Without the presence of a caretaker, the Bridge House suffered from vandalism. Eventually, all the original glass panels broke, were removed, and replaced with plywood and barriers to prohibit entrance. During this time, the Bridge House was used as a storage facility (Planas, 2011).
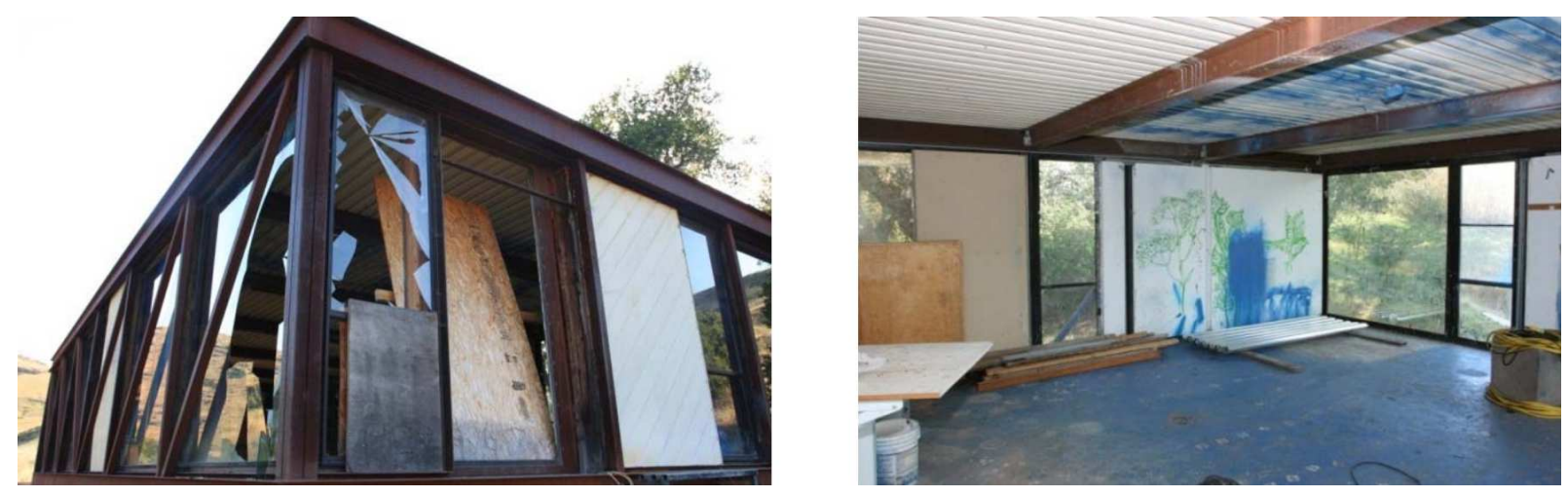

Figure 2-8: Bridge House circa 2011 (Planas, 2011)

\subsubsection{Structural Dynamic Field Laboratory, 2011 - Present Day}

In 2011, another group of Architectural Engineering students took on a senior project to work on the Bridge House. Their efforts were two-fold. First, they wished to turn the building into a dynamics experimental laboratory by adding removable braces to Bridge House in the North-South direction. These braces turned the Bridge House into a one-of-a-kind structure as 
it allowed students to change the building properties of the structure. The lateral stiffness of the Bridge House became variable depending on the respective brace configuration. Second, they aimed to restore and clean up the Bridge House to bring it up to acceptable conditions. They gutted and cleaned the interior of the structure.

By 2013, all the exterior glass of the Bridge House had been broken. Plywood panels were added to keep intruders out of the structure. Despite these panels, intruders continually made it into the structure. Reinforced barriers made of an assortment of materials were added to certain locations. Student and faculty research in the Bridge House was conducted during this time. Irregularities in the roof diaphragm behavior lead researchers to believe that the corrugated roof decking was never properly adhered to the roof wide flanges. In the spring of 2014, two students epoxied the wide flange roof beams and the corrugated desk at two foot intervals. The goal of this modification was to force regular diaphragm behavior.

In the spring of 2015, the College of Architecture and Environmental Design initiated efforts to rehabilitate Poly Canyon. Plans called for the removal of all elements not original to the structure. Guests to Poly Canyon would not be allowed to roam and explore the Bridge House with its newly restored views. The addition of handrails were necessary for safety. An up-to-date picture of the Bridge House is shown below. 


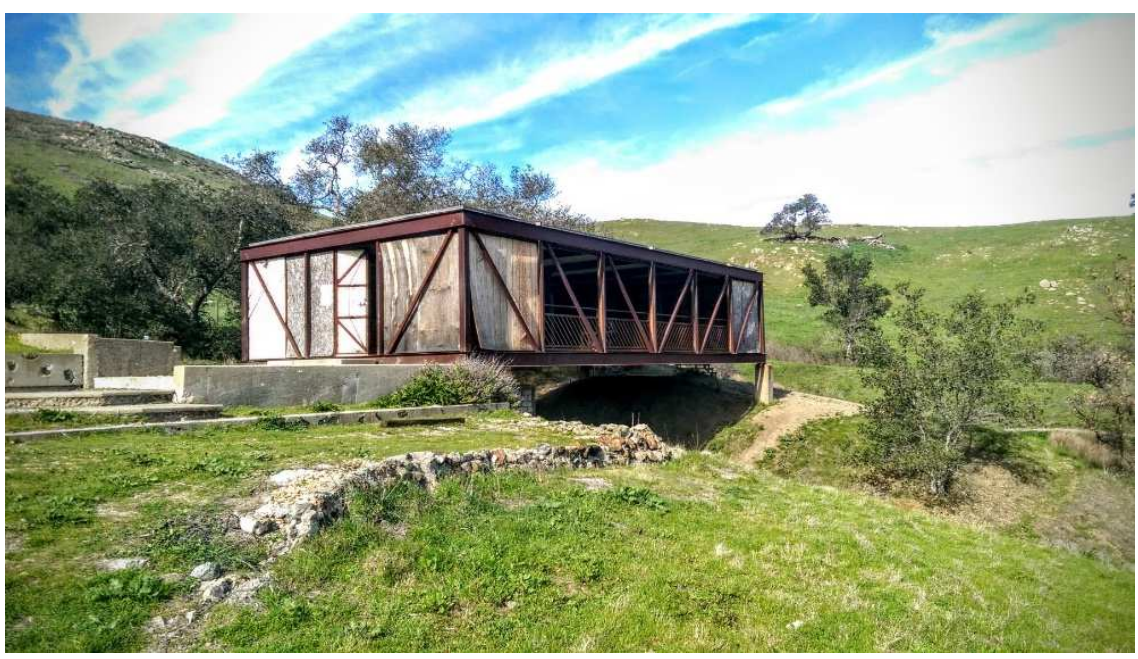

Figure 2-9: Bridge House in current state

\subsection{Building Survey}

A building survey was necessary to grasp greater understanding of the characteristics, the as-built conditions, and the modifications made to the Bridge House over the its history.

\subsubsection{Original Plans and Survey}

Plans, calculations, and background papers about the Bridge House are available in university archives. The drawings closely match the as-built conditions with a few modifications. Braces on the ends of the Bridge House were in the original drawings but were never installed.
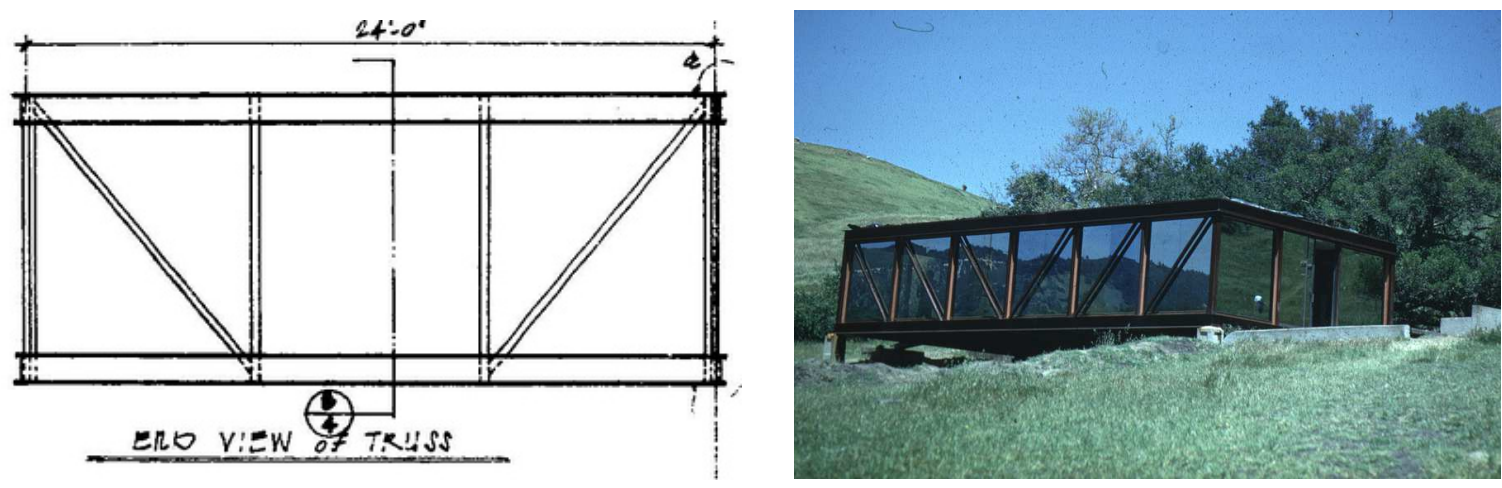

Figure 2-10: Original plans and as built 
The other major structural change occurred at the interior beams. The drawn detail showed a bolted shear tab. All as-built conditions were observed as welded shear tabs. At the foundation, a bolted base plate design was shown in original plans. No bolts are observed, therefore, it is assumed that embedded studs, j-bolts, or rebar was used instead. Additionally, all non-structural steel (i.e. window mullions) were never included in the original plans. Other differences were observed but were mainly small details regarding insulation, roofing, and mullion details.

\subsubsection{Exterior Survey and Structural System}

The top and bottom chords of both the Vierendeel truss and the brace frame are double C12x20.7 channels. The back of the channels are spaced approximately 3 inches apart to facilitate the welding of the truss diagonals and the exterior columns to the back face. The typical columns are HSS $3 \times 3 \times 1 / 4$ welded top and bottom to the double channels. However, they can more accurately be classified as a built-up section as the steel window mullions add significant stiffness. The built-up section of the steel window mullion to the HSS interior columns is stich welded at various increments.
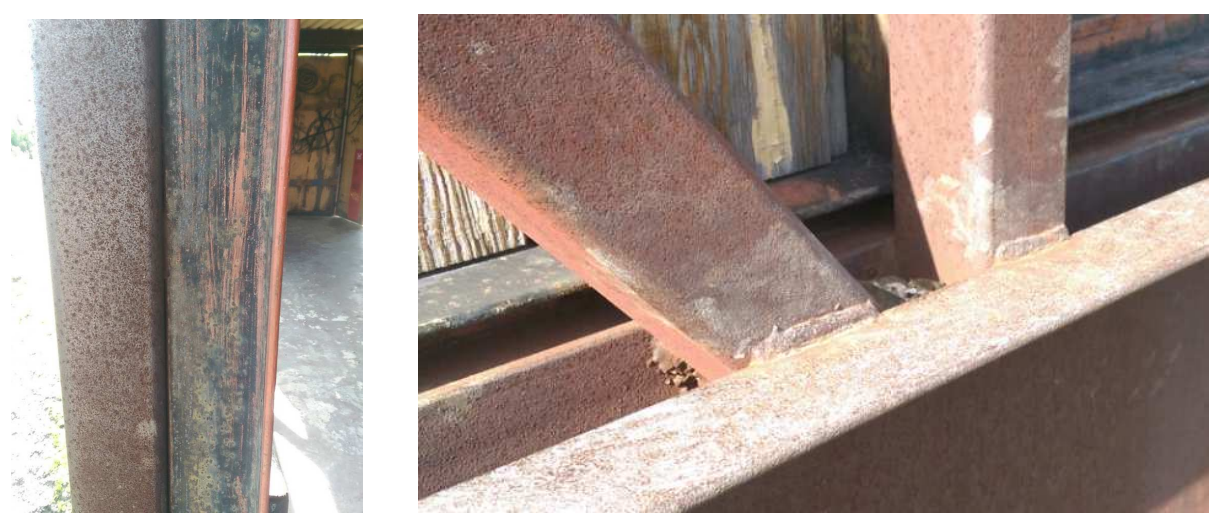

Figure 2-11: Built up column (left), column detail (right) 
The corner columns are built-up sections composed of four HSS $3 \times 3 \times 1 / 4$. The HSS sections are butted together with no intermediate welds; the only connection of each built-up member occurs at the end where they are abundantly welded.
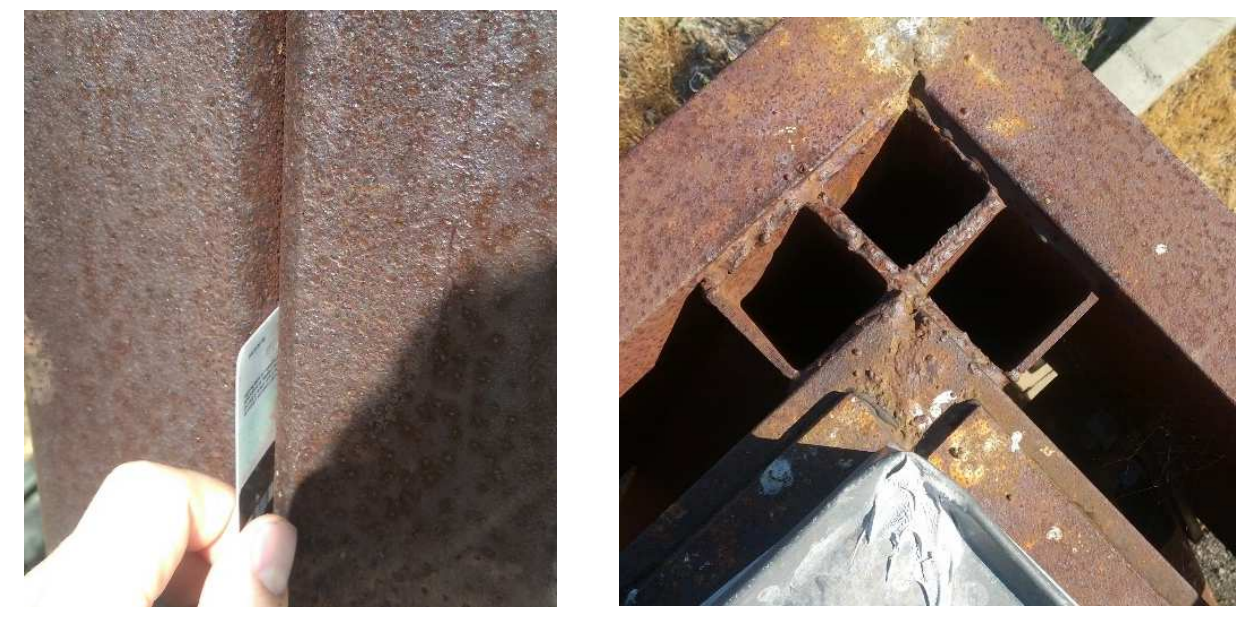

Figure 2-12: Corner column detail

The interior beams that span the floor and the roof deck are W8x31. They are welded to the chords of the trusses by shear tabs. Steel roof decking spans between the W8x31 roof and floor beams.

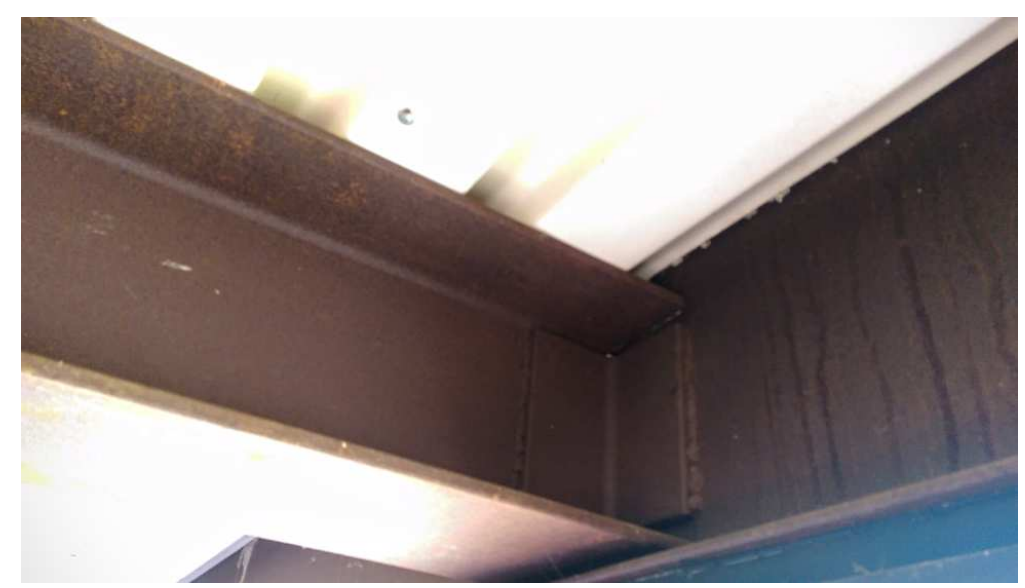

Figure 2-13: Wide flange roof detail 
Unlike typical bridge structures, the Bridge House has no expansion or thermals gaps to allow for expansion and contraction. Any stresses that are induced due to thermal effects are not able to be released.

\subsubsection{Roof System}

Previous research identified the mass of the roof by saw cutting, removing, and weighing a square foot of the roof section. The composition was determined to be a rigid insulation and weatherproofing material with gravel topping. This section was determined to weigh fifteen pounds-per-square foot. To determine the stiffness of the deck, the underside of the roof deck was investigated. These results were compared to old catalogues and the deck was determined to be Robertson Sec. 3 decking. The thickness of the deck was determined to be 18 gauge after a small inspection opening was made and the thickness was measured.

Roof deck seams that run parallel to the beams are visible every two feet. The length of the panels is assumed to be eight feet long. This is consistent with product availability and historic photos of the Bridge House; however, there is no evidence to suggest that the seams of adjacent panels are connected or adhered together.

Additionally, it is unknown if, or how, the roof deck was originally connected to the rest of the structural system. Typically, puddle welds would be used to attach the deck to the roof beams. Burn marks on the underside would be evidence of welds. There are no burn marks or any evidence to suggest that roof deck is adhered to the roof beams.

Lastly, it is believed that the roof deck is not properly adhered to the double channels external beams that surround the roof. These double channels would act as chords and collectors in a flexible roof system. An inspection camera was utilized to examine the boundary conditions of the roof deck to the rest of the structure; no such connection was found. 
These issues were a concern for previous research done on the Bridge House (Ramos, 2013). Specifically, the researchers were concerned whether the steel deck was adhered to the roof beams and a friction connection was the only way forces were transferred. To mitigate these issues, the roof beams were epoxied to the roof beams at two foot intervals.

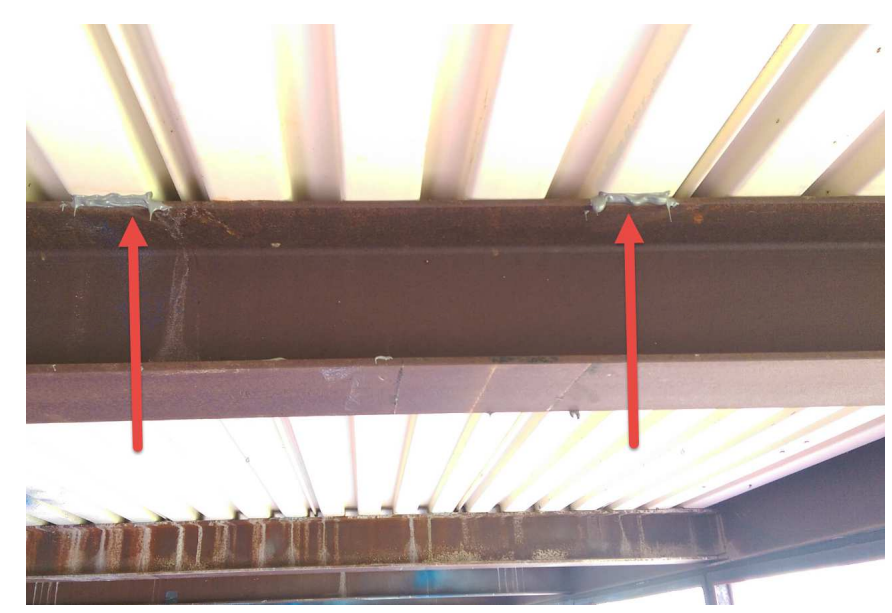

Figure 2-14: Epoxied roof

\subsubsection{Floor System}

The floor of the Bridge House is a concrete topping slab above corrugated decking. Bellow the corrugated decking, pan metal cold sheets are spot welded to the deck making an underside inspection unfeasible. Therefore, several inspection openings were drilled into the concrete deck to measure the deck at several different locations. It was determined the floor deck was the same as the roof, Robertson Sec. 3 and because it was not possible to determine the gauge, it was assumed it to be 18 gauge as well. The deck was topped with approximately $1-11 / 16$ inches of concrete. The weight of the concrete is unknown. The original plans called for lightweight concrete and it is assumed that lightweight concrete was installed. 


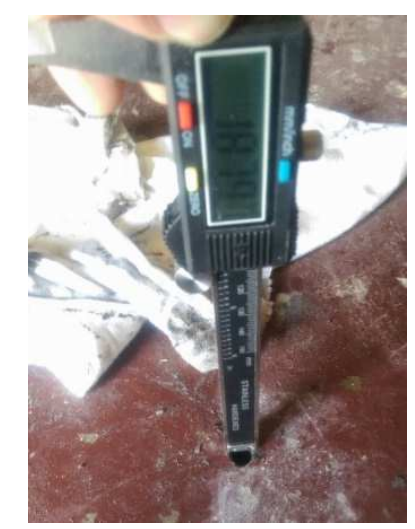

Figure 2-15: Floor inspection hole

\subsubsection{Substructure}

The Bridge House sits on four concrete piers at each corner. The original plans call for footings with pedestals. Because the piers are submerged bellow the soil, the geometry of each pier is unknown. Above ground, a visual inspection shows that each pier is exposed at different elevations. Some piers have approximately four feet of exposed concrete, others are completely submerged. Each pier's total height, as well as embedment is also unknown.
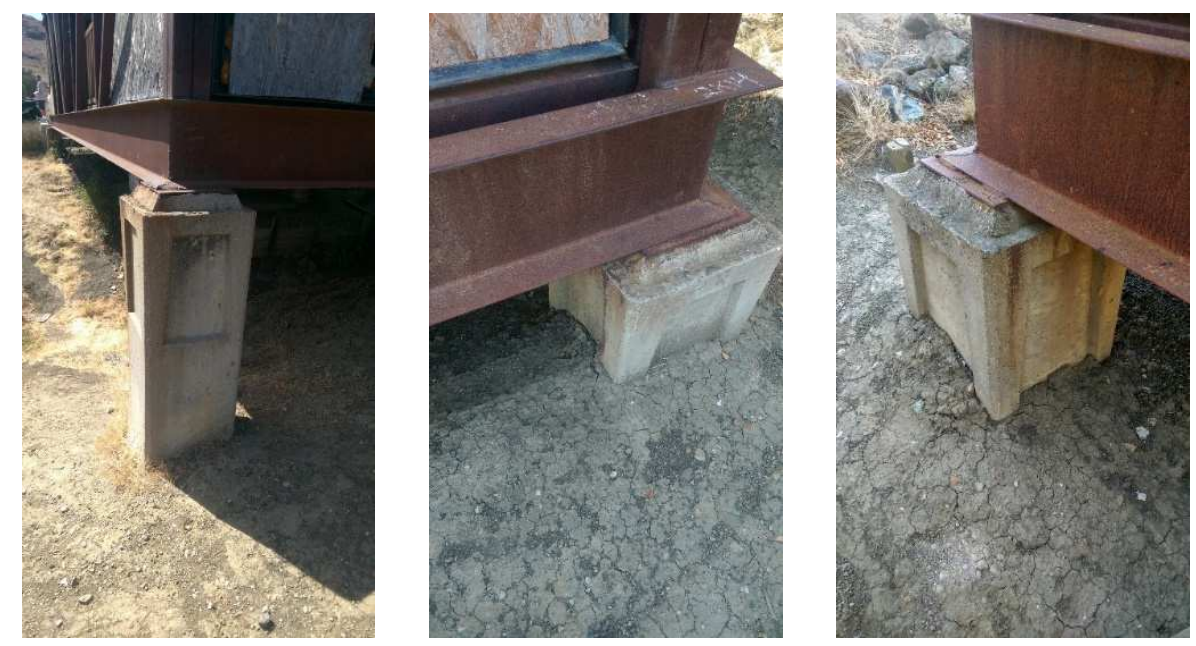

Figure 2-16: Examples of the various piers at the Bridge House 


\subsection{THEORY AND METHODOLOGY}

\subsection{Overview}

It is assumed that steady state response, modal analysis, the equation-of-motion, and a system subjected to sinusoidal forces are previously well understood by the reader. Discussions here are a summary of the importance of steady state response. Detailed derivations and explanations are listed in Appendix 0. Traditionally, dynamic response would also have transient homogenous behavior. This will be ignored as the contribution during steady state is small and irrelevant. It will be shown in the following sections that the response due to forced vibration at resonance is much larger than transient behavior.

The equations in this section are for a single-degree-of-freedom (SDOF) system. Additional assumptions are needed for a multi-degree-of-freedom system (MDOF). In a dynamic analysis, the response of the structure is made up of many equations-of-motion (EOM) that are coupled together. A modal analysis decouples the EOM into independent and orthogonal SDOF equations. If the system is first mode dominate, the dynamic properties can be idealized as a SDOF system. When coupling the EOM back into a MDOF system, if the contribution of higher modes is small, the system can be modeled as a SDOF system.

\subsection{Displacement Response Amplification Factor, Rd}

The displacement response amplification factor, $R_{d}$, is a ratio that compares the static deformations to the dynamic deformations of similar systems. The displacement response amplification factor is as follows:

$$
R_{d}=\frac{u_{\max }}{\left(u_{s t}\right)_{0}}
$$

Where $\left(u_{s t}\right)_{0}$ is the deformation due the applied force and $u_{\max }$ is the maximum displacement of a structure when subjected to a sinusoidal force. 


$$
\begin{gathered}
\left(u_{s t}\right)_{0}=\frac{p_{0}}{k} \\
u_{\max }=\frac{p_{0}}{k} \frac{1}{\sqrt{\left[1-\left(\frac{\omega}{\omega_{n}}\right)^{2}\right]^{2}+\left[2 \zeta\left(\frac{\omega}{\omega_{n}}\right)\right]^{2}}}
\end{gathered}
$$

Substituting Equation 3-2 and Equation 3-3 into Equation 3-1 cancel outs $p_{0} / k$ and yields the following equation at steady state:

$$
R_{d}=\frac{1}{\sqrt{\left[1-\left(\frac{\omega}{\omega_{n}}\right)^{2}\right]^{2}+\left[2 \zeta\left(\frac{\omega}{\omega_{n}}\right)\right]^{2}}}
$$

Plotting Equation 3-4 for a variety of damping ratios of an excited system is show in Figure 3-1. 


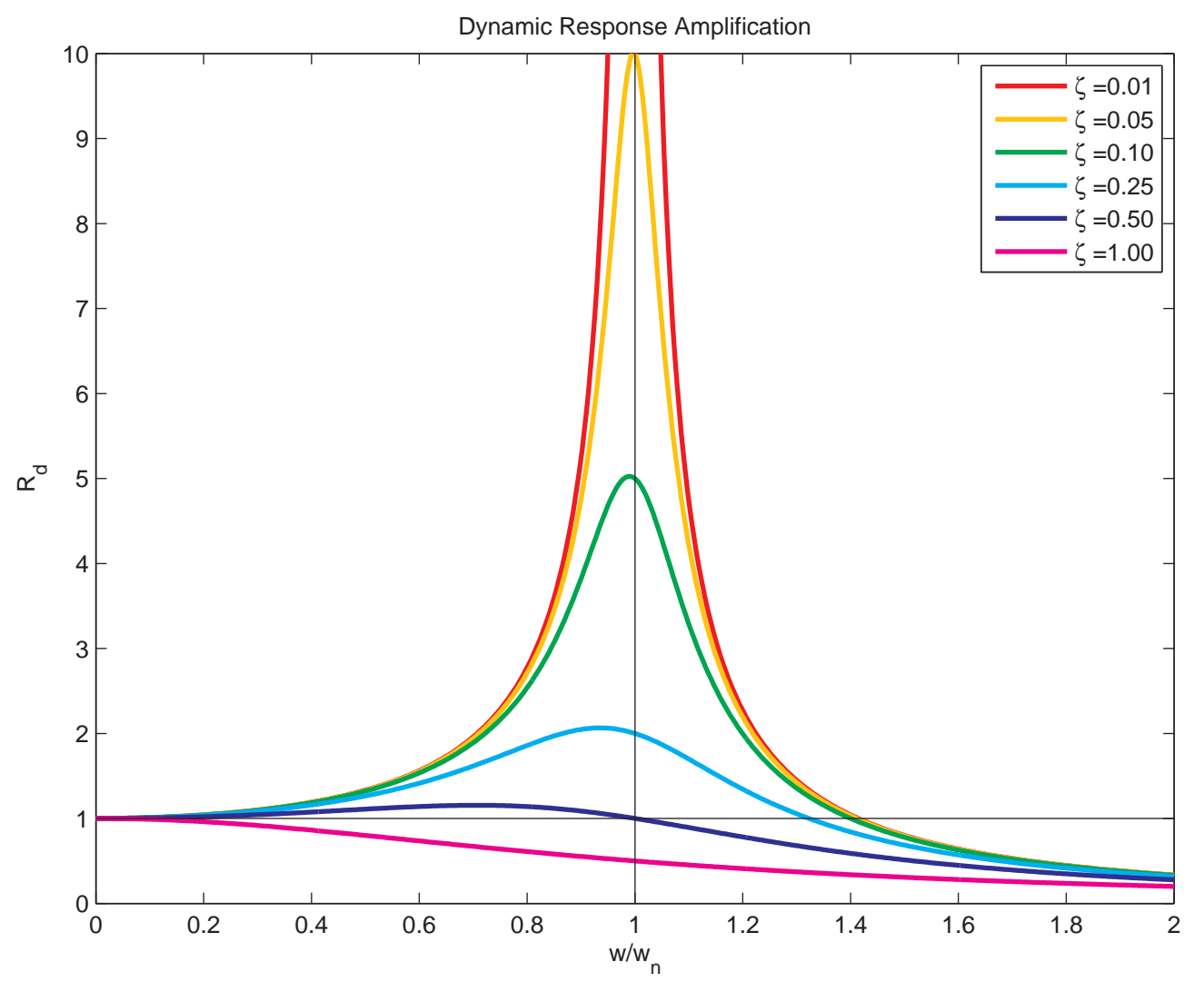

Figure 3-1: Dynamic Response Displacement Amplification

Increasing damping for a system will reduce the displacements. For low-dampened systems, (when $\zeta$ is approximately less than 10 percent), the maximum displacements occur when the system is excited at its natural frequency (when $w / w_{n}=1$ ). For systems with large damping, the maximum displacements will not occur at $w / w_{n}=1$. For typical building systems, zeta is well below 10 percent. Therefore, for the scope of this research, it shall always be assumed that the maximum displacement occurs when $w / w_{n}=1$. By exciting a building at a variety of frequencies, a response amplification plot as shown in Figure 3-1 can be composed. The frequency-displacement response curve can be calculated experimentally by subjecting a building to a sinusoidal dynamic force, waiting for steady state response, and measuring the acceleration response. This is repeated for several frequencies until an experimental frequency-acceleration response curve is built. 


\subsection{Damping}

To experimentally calculate damping ratios, two methods can be utilized. One method utilizes the transient response properties due to the decay of motion. This method typically involves enforcing a displacement in the building, releasing the displacement, and recording the decay of the vibrations. By recording the time history, damping can be calculated. Because of the size and terrain limitations of the Bridge House, this method cannot be utilized. A second method called the Half-Power Bandwidth allows for the calculation of the damping from a displacement response amplification curve.

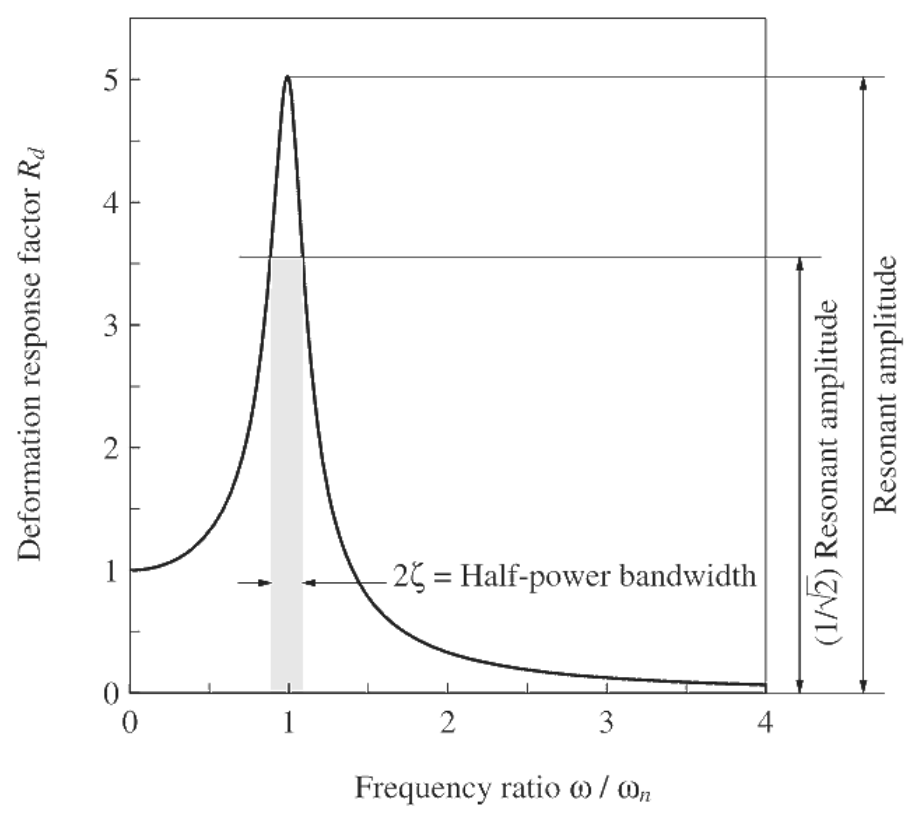

Figure 3-2: Damping calculation (Chopra)

The half-power bandwidth method allows the calculation of the damping from the displacement frequency response curve. Simplification and substitution yields the following:

$$
\zeta=\frac{f_{b}-f_{a}}{2 f_{n}}
$$

Where $f_{b}-f_{a}$ is defined as the bandwidth and $f_{n}$ is the natural frequency. In lieu of frequencies, periods may be used to calculate the damping ratio. 


\subsection{TESTING PROCEDURE AND SET-UP}

\subsection{Overview}

Experimental mode shapes must be acquired to compare results to theoretical models. The experimental procedure will consist of shaking the Bridge House with a large oscillating mass and recording the response of the building through the use of strategically placed accelerometers.

\subsection{Equipment}

The equipment to perform the testing is broken up into two independent systems. One system is used to excite the building for forced vibration testing. The second system is a data acquisition system that records accelerations.

\subsubsection{Forced Vibration Equipment}

To excite the building, three pieces of equipment are needed: a variable signal generator, a signal amplifier, and a mechanical shaker. The sequential block diagram is shown in Figure 4-1.

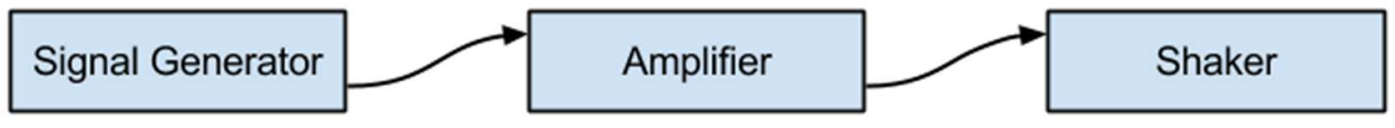

Figure 4-1: FVT block diagram

The signal generator used is a BK Precision 4084. It is capable of producing a clean sine signal at multiple amplitudes and frequencies. The output from the signal generator was amplified by an APS 125 Power Amplifier and fed into an APS 113 Long Stroke Shaker. The shaker is a linear bearing shaker that is capable of producing a maximum of a 30 pound force. Previous internal research indicated the shaker produces just under thirty pounds of force when 
the signal generator is running at 2.0 volts-peak-to-peak (Gerbo 2014). This force remains constant over the range of frequencies typically used to test a building.
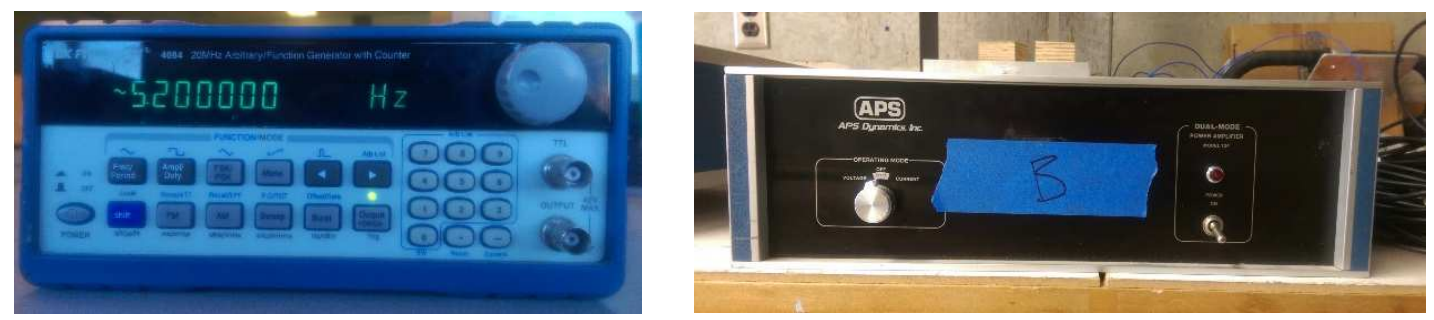

Figure 4-2: Signal generator, amplifier

The Bridge House has a shaker permanently installed at the underside of the center of the roof. This shaker is capable of exciting predominately translational mode shapes.

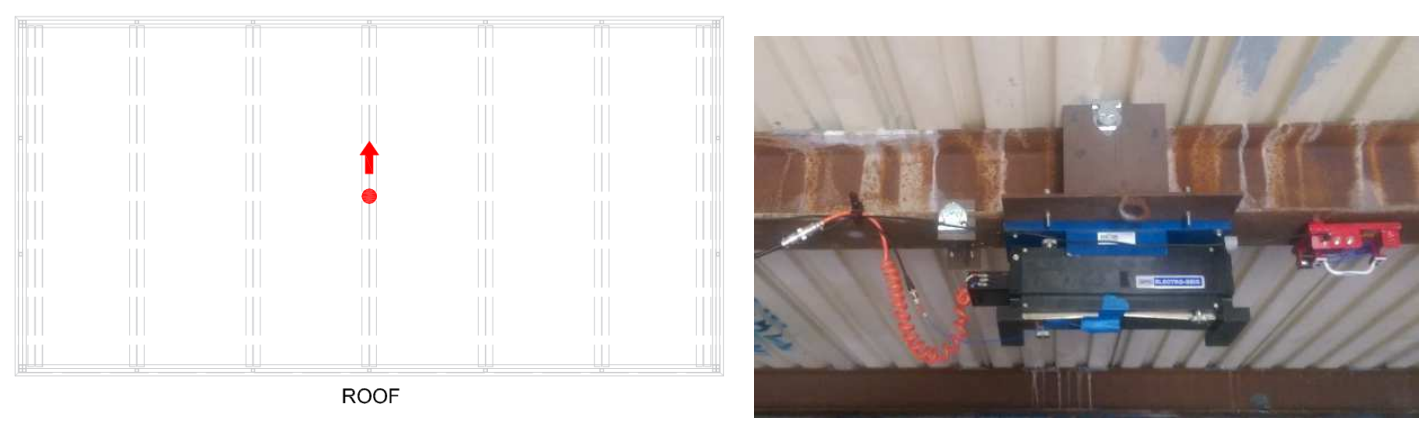

Figure 4-3: Shaker location

\subsubsection{Data Acquisition}

To record the building behavior, ten PCB 393B04 accelerometers were used with two National Instruments 9233 data acquisition units and one National Instruments 9234 data acquisition unit. All signals were acquired with National Instruments Signal Express. Within the program, basic signal processing was done. The steps of this process are outline in Figure 4-4. 


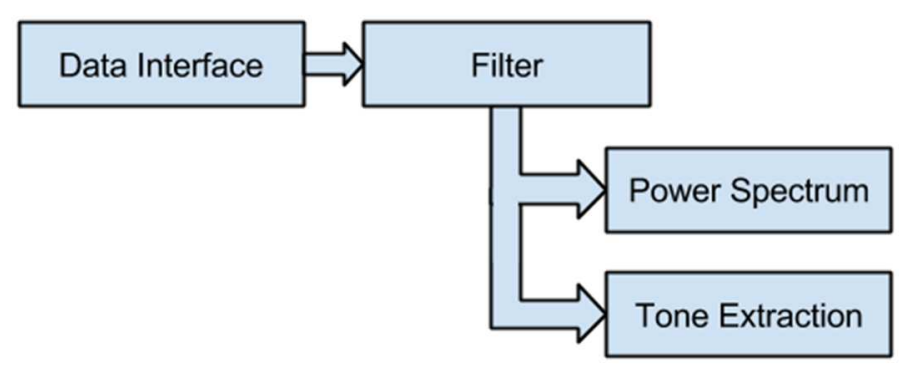

Figure 4-4: Data processing

When necessary, a filter was applied to the data. A power spectrum analysis and tone extraction was done that outputs the detected frequency, the respective amplitude, the respective phase, and a frequency-domain plot. The tone extraction and power-spectrum analysis were done with a Fast-Fourier-Transform (FFT).
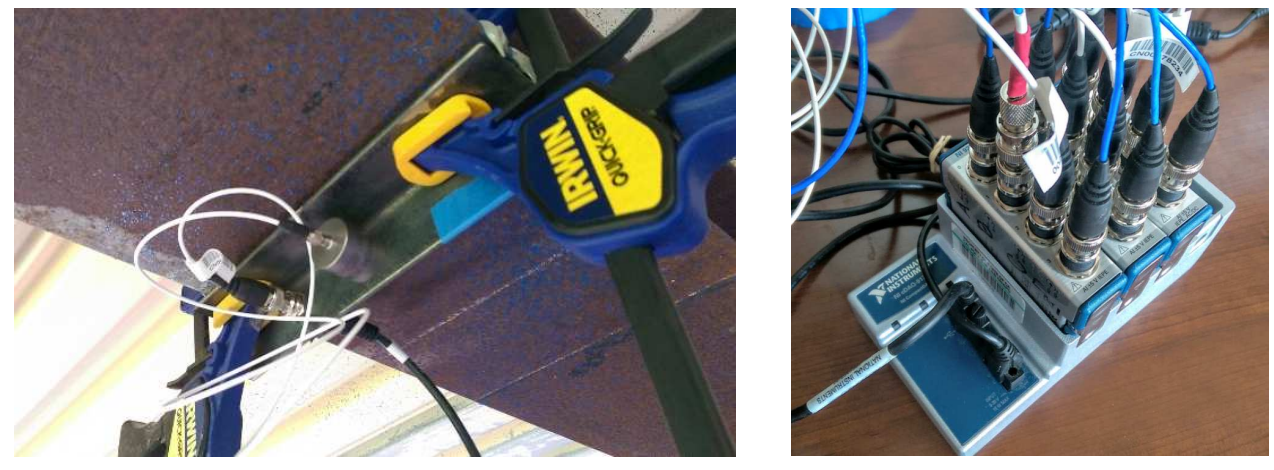

Figure 4-5: Data acquisition equipment

Seven accelerometers were placed on the roof and two were placed on the floor. Two accelerometers can capture the behavior of rigid body behavior. The assumption of rigid floor behavior will be examined subsequently, and for simplifying analysis, the accelerations from the two floor accelerometers will be extrapolated to seven accelerations. One additional accelerometer was placed on the shaker to ensure regular performance. 


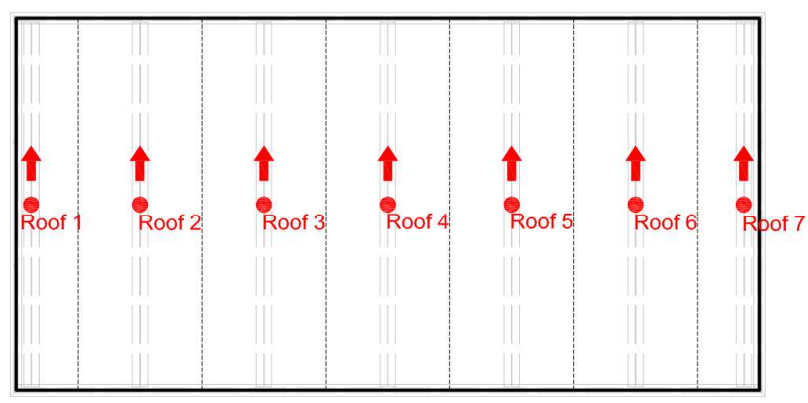

ROOF

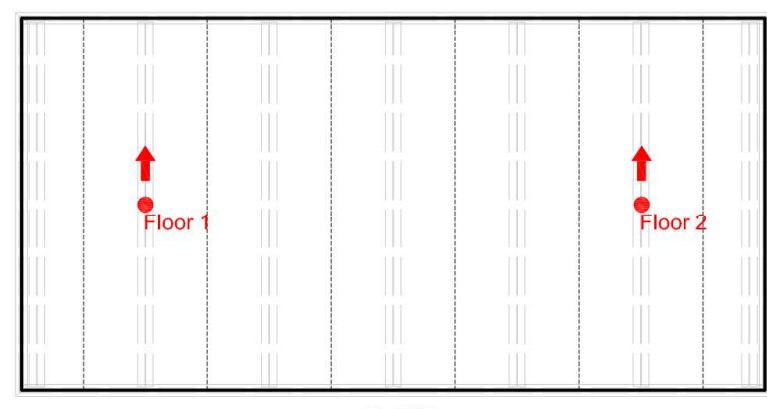

FLOOR

Figure 4-6: Location and direction of accelerometers

\subsection{Testing Procedure}

The data acquisition procedure is broken into four steps with two different test methods. The four steps are an ambient vibration test, a wide forced vibration sweep, a narrow forced vibration sweep, and steady state measurement at resonance.

\subsubsection{Ambient Vibration Testing}

An ambient vibration test consists of taking measurements of ambient noise; data acquisition is done with the shaker at rest. At rest, buildings tend to deform in their mode shapes due to ambient forces such as wind and exterior motion. By examining the extracted amplitudes over several trials, an estimate can be made as to the frequency of the first mode.

\subsubsection{Forced Vibration Testing}

The first forced vibration test performed is a wide sweep. The shaker is set to output a range of different frequencies. During this time, the absolute peak accelerations, along with the respective frequency of the shaker, are recorded and plotted. This plot is an experimental response amplification chart; the peaks on the chart represent the natural frequencies and the mode shapes. Once a mode shape has been identified, a narrower sweep is conducted until the highest acceleration is found. The forcing frequency that corresponds to the highest accelerations indicates the natural frequency of the structure, and the structure is at resonance. 
At this point, the shaker frequency is fixed and acceleration readings are taken. These acceleration readings correspond to the experimental mode shapes. These procedures were repeated several times for different shaker amplitude and brace configurations. For the scope of this thesis, only first mode from each brace configuration will be examined.

\subsubsection{Brace Configurations}

The Bridge House has four removable braces, each having a binary state, engaged or disengaged. An engaged brace represents a brace that is undamaged; a disengaged brace represents a brace that has ruptured, or is damaged and offers no lateral resistance.
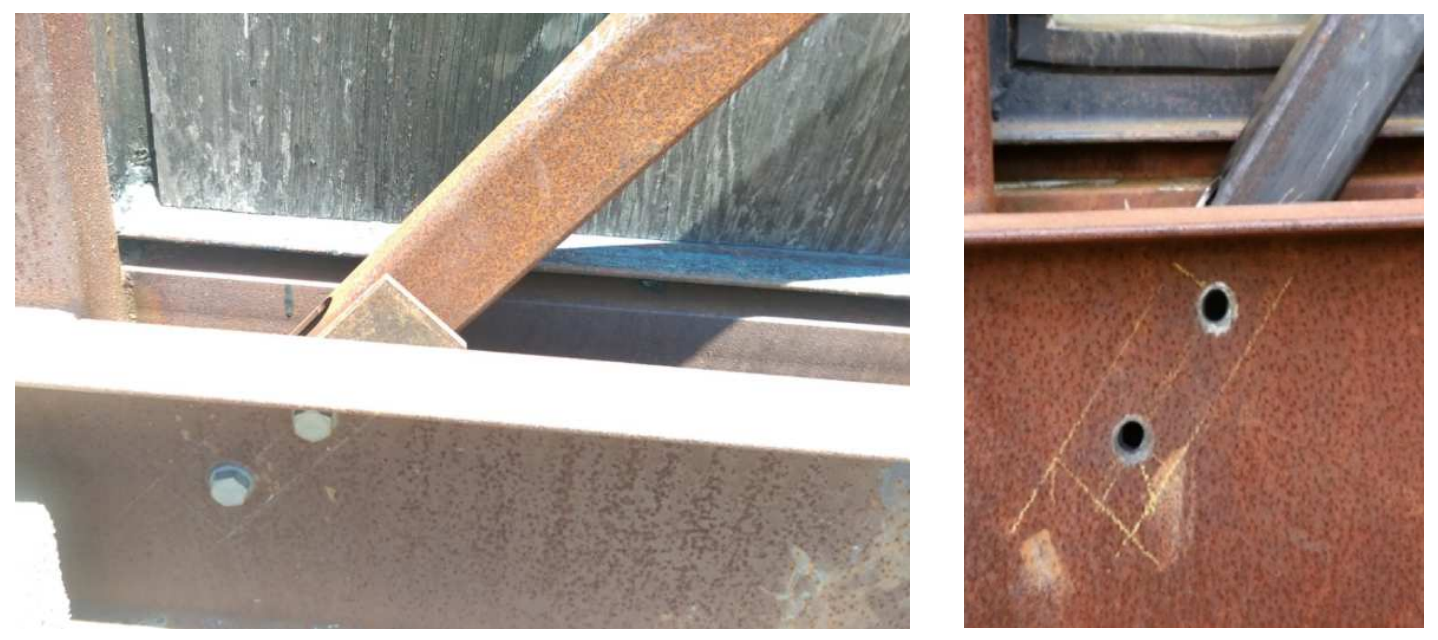

Figure 4-7: Removable braces

Four elements, with two different states, yield sixteen total brace configurations. If either brace within a line is damaged, the results would effectively be very similar and the number of unique brace configurations is less than sixteen. For the scope of this research, four brace configurations will be examined. These are All Braces On, No Brace, West Braces Only, and East Braces Only. 


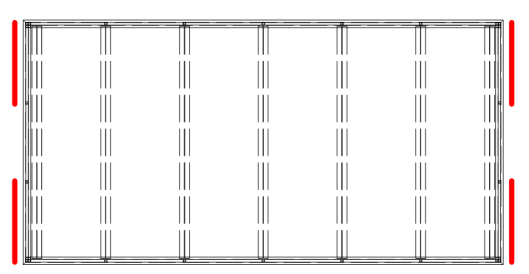

All Braces On

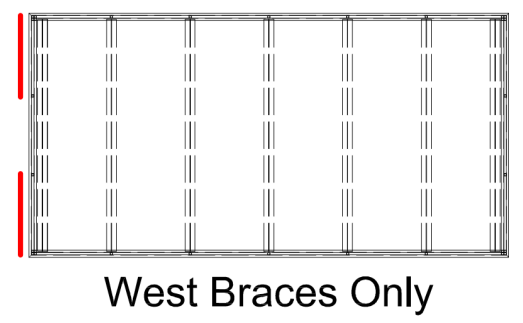

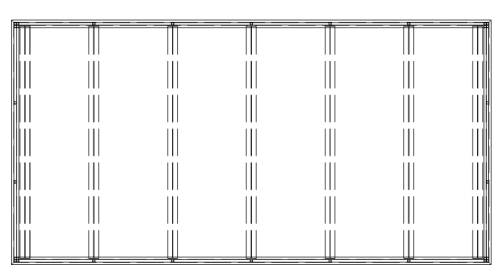

All Braces Off

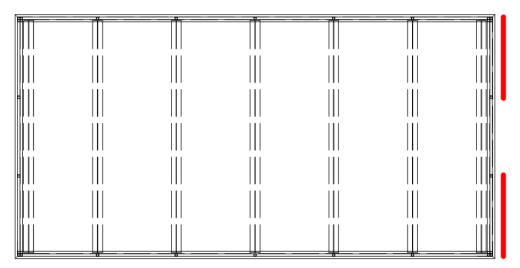

East Braces Only

Figure 4-8: Brace configurations 


\subsection{TESTING RESULTS AND SYSTEM IDENTIFICATION}

\subsection{System Identification}

System identification of a building consists of analyzing a structure to extract the structural properties of the building. Typically, system identification by the use of forced vibration testing consists of determining the frequency response spectrum, the mode shapes, and the damping. Through the use of dynamic principals and equilibrium, the stiffness of the braces, the roof diaphragm, and the substructure can also be determined.

\subsection{Ambient Vibration Test}

Ambient vibration tests were forgone for the Bridge House. Prior trial tests established the approximate fundamental frequencies and eliminated the need to search for the range of excited frequencies.

\subsection{Forced Vibration Sweep}

Force vibration sweeps were done to construct the response amplification graphs for the Bridge House. The accelerometer used to construct the response amplification is shown in Figure 5-1. A single location suffices in building a response amplification graph for the translational mode shapes at the Bridge House. Had translational modes been examined, multiple locations would be necessary.
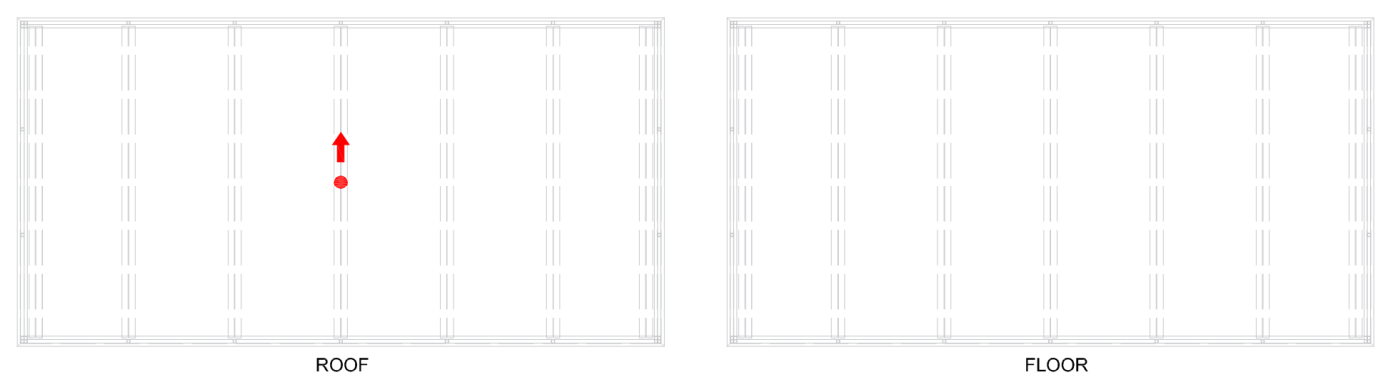

Figure 5-1: Location and direction of accelerometer used for FVT sweep 


\subsubsection{Variation}

The Bridge House was tested several times. Each test used the same shaking parameters but at various times on different days. Several different tests yielded different results. To compare tests to one another, each test was infinity normalized to one. The results from different days are shown in Figure 5-2.
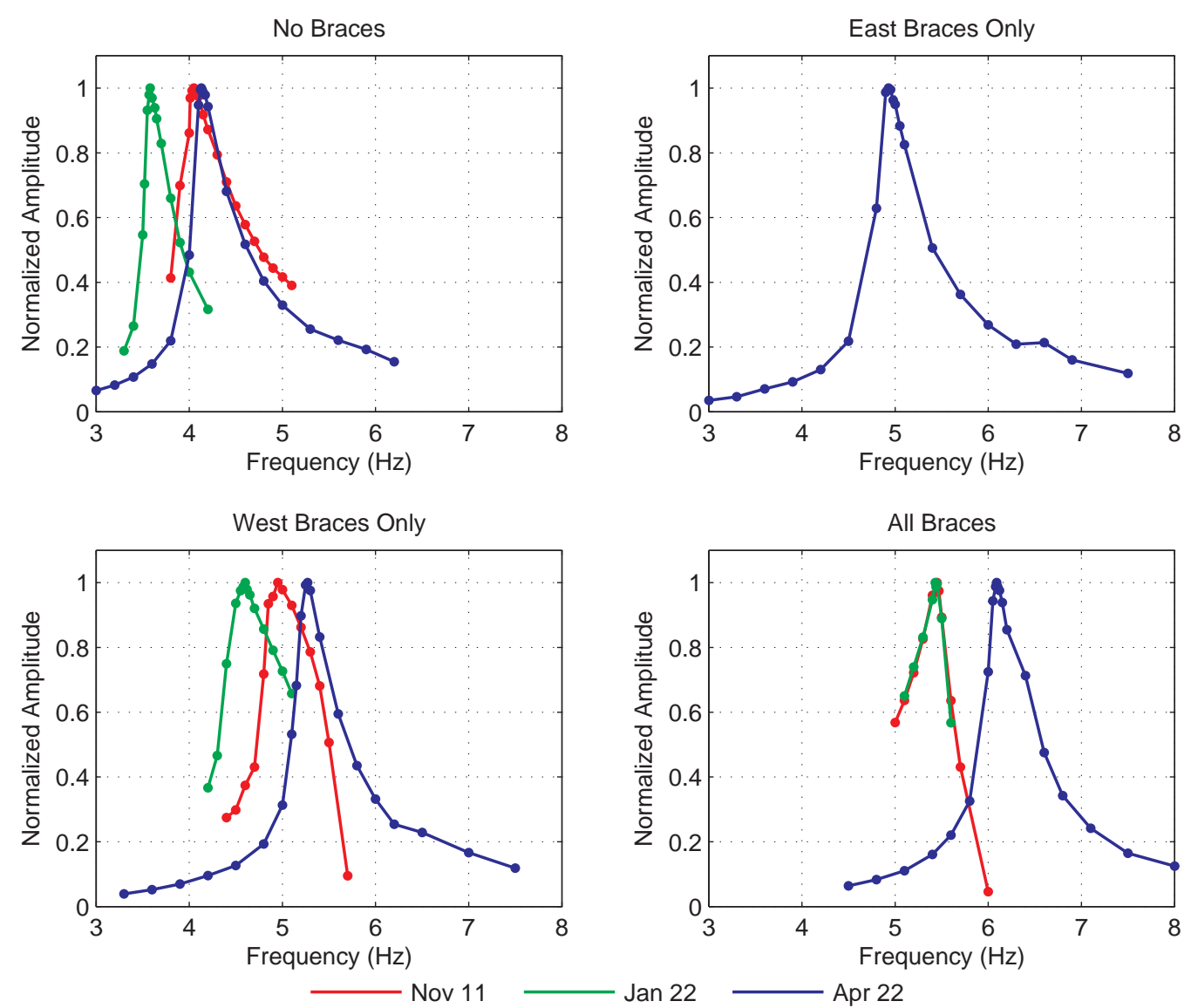

Figure 5-2: Varied response amplification for multiple trials

The results above indicate variance in the response of the Bridge House. It was discovered that the response of the structure could change over the course of a small amount of time (less than an hour). If the response of the structure unknowingly changes, the testing may not be at resonance, and the recorded accelerations may no longer be pure first mode behavior. 
Typically, minor adjustments in the response amplification are ignored in forced vibration testing; the deviations observed in the Bridge House were not minor. This discrepancy has been well documented in the past (Ramos, 2013). It has been observed that the Bridge House is susceptible to temperature effects as the structural system is exposed steel. Some parts of the Bridge House are exposed to direct sunlight while others are subjected to complete shade. A surface temperature gradient on the steel has been measured as large as 100 degrees Fahrenheit.

To solve this problem, two changes to the procedure were made. First, more accelerometers were acquired to expedite the testing and to facilitate concurrent data acquisition. Second, all testing was done at night to eliminate environmental variables. These changes allowed for repeatable and consistent results that were verified through multiple trials. The results are shown in blue in Figure 5-2.

\subsubsection{Final Forced Vibration Sweep}

After procedural changes were made, a final forced vibration frequency sweep was conducted. The final for each brace configuration are shown in Figure 5-3. It is important to note that the amplitude has not been normalized for each brace configuration and the output of the shaker is a fairly constant at 30 pounds. The maximum accelerations for each brace configuration varies; the accelerations were recorded at the center of the Bridge House as shown in Figure 5-1. For this reason, the magnitude of the final force vibration sweep should not be regarded as significant. 


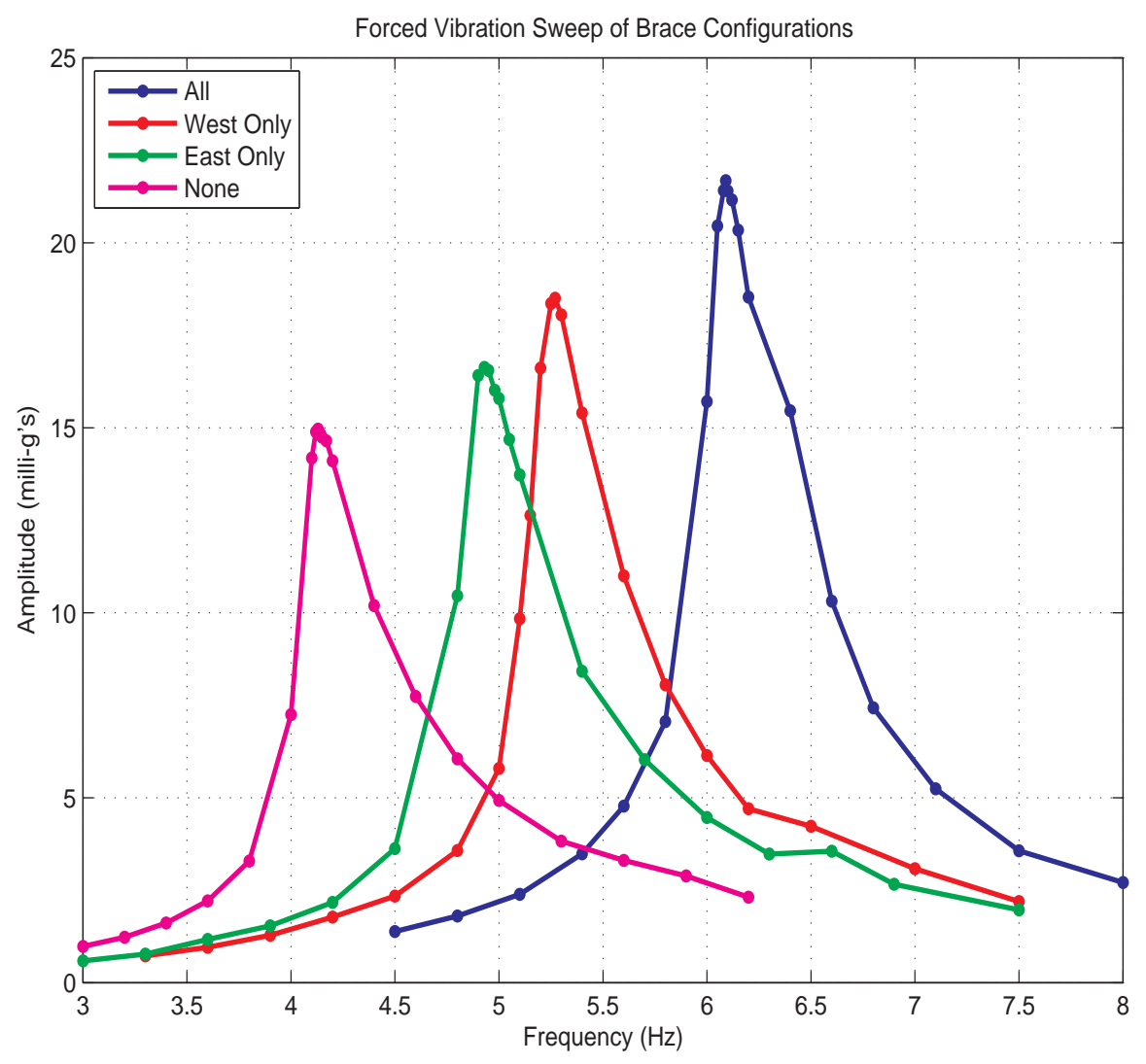

Figure 5-3: Final experimental response forced vibration sweep

The peak of each plot represents the frequency of the first mode for each brace configuration. The respective frequencies are shown in Table 5-1. As expected, the more the braces that are engaged, the higher the frequency. It is expected that the West Only brace configuration should be slightly higher than the East Only brace configuration as the East side of the Bridge House has stiff non-structural elements. This is observed in the experimentation results.

Table 5-1: Experimental frequency of first modes

\begin{tabular}{|c|c|}
\hline Brace Configuration & Frequency $(\mathrm{Hz})$ \\
\hline All & 6.09 \\
\hline West Only & 5.27 \\
\hline East Only & 4.93 \\
\hline None & 4.13 \\
\hline
\end{tabular}




\subsection{Damping}

Using the half-power bandwidth method as shown in Figure 3-2, the experimental results for the various brace configurations are shown below.

Table 5-2: Tested damping coefficients

\begin{tabular}{|c|c|}
\hline Brace Configuration & Damping \\
\hline All & 4.04 \\
\hline West Only & 3.03 \\
\hline East Only & 3.95 \\
\hline None & 2.99 \\
\hline
\end{tabular}

The values are all within expected damping values of a steel structure. These damping values are also within the ten percent threshold discussed in section 3.2 ; the assumption that max displacements and max accelerations occur when $w / w_{n}=1$ holds valid. There is an increase in damping when less braces are engaged. This is expected as the external cladding elements along the brace line are engaged, although it is difficult to show causation. These cladding elements closely resemble damage; as damage increases in a building, the damping increases as well.

\subsection{Mode Shapes}

\subsubsection{Rigid Floor Verification}

The as built condition of the Bridge House is not completely known. Verification of the floor behavior is necessary. To verify the floor behavior, seven accelerometers were placed on the floor and a forced vibration test at resonance was performed. Several trials yielded similar results. The results of a typical trial are shown below in Figure 5-4. 
Floor Accelerations

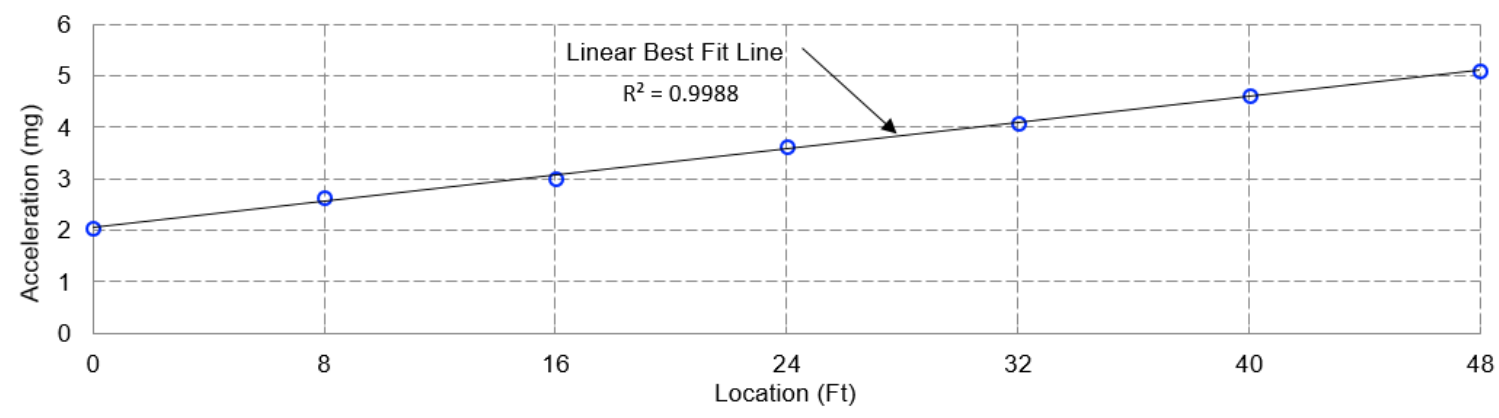

Figure 5-4: Experimental floor behavior

Testing shows linear results which indicates the floor behaves as a rigid body. Two irregularities were spotted at 8 and 16 feet. The cause of these irregularities is unknown and has little to no impact on the test results. In addition, this verification allows the testing to be conducted with less degrees-of-freedom. Because the floor behaves as a rigid body, two accelerometers can capture rotation and one direction of translational behavior. To achieve additional degrees of freedom, the behavior of the two degrees-of-freedom can be extrapolated. Having additional degrees of freedom eases analysis for several reasons. It allows for increased precision when making a lumped mass assumption and easier calculations when computing story drift.

\subsubsection{Raw Mode Shapes for Multiple Shaker Voltage Input}

After several pre-trail runs, a final data collection of experimental mode shapes was collected. For every brace configuration, the shaker was set to four different peak-to-peak voltages: 0.5 VPP, 1.0 VPP, 1.5 VPP, and 2.0 VPP. 
All Braces Roof

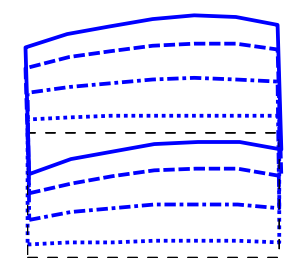

No Braces Roof

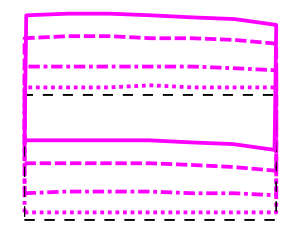

West Braces Only Roof

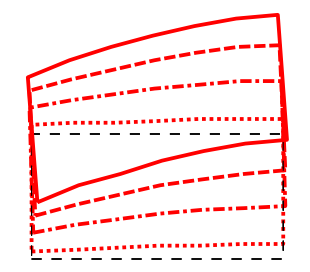

East Braces Only Roof

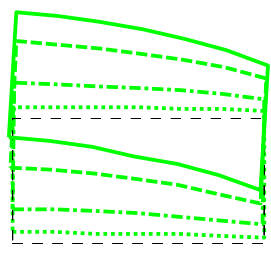

All Braces Floor

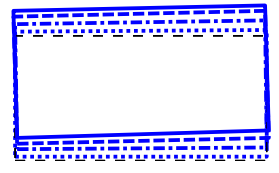

No Braces Floor

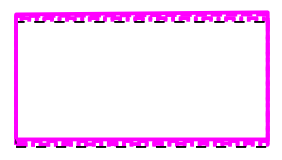

West Braces Only Floor

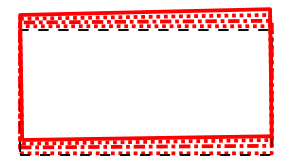

East Braces Only Floor

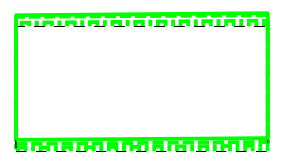

Undeformed Shape $0.5 \mathrm{VPP}$ $1.5 \mathrm{VPP}$ $2.0 \mathrm{VPP}$

Figure 5-5: Experimental mode shapes for each trial

Displacements increased proportionally to shaker voltage. By comparing the floor behavior to the roof behavior, a few distinct observations can be made. For the No Brace configuration, the floor displacements are much smaller than the roof compared to other brace configurations. Conversely, the floor displacements in the All Braces On configuration are much greater than the All Braces On. These behaviors are expected due to the stiffness distribution of each brace configuration. 


\subsubsection{Normalized Mode Shapes}

To compare the mode shapes of each brace configuration for various shaker amplitudes, a normalization of the mode shapes was done. Each voltage trial for each brace configuration was infinity normalized to unity.

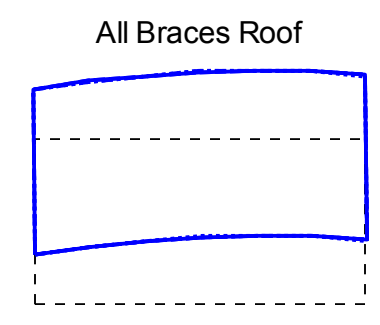

No Braces Roof

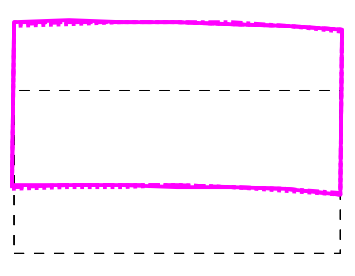

West Braces Only Roof

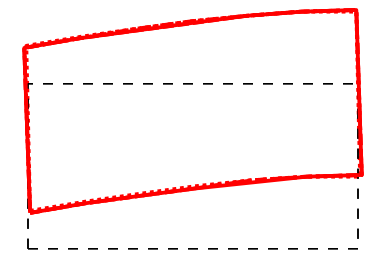

East Braces Only Roof

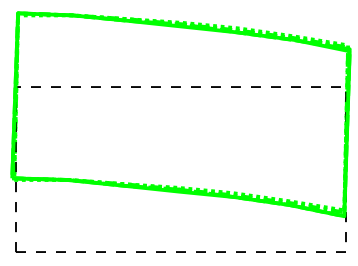

All Braces Floor

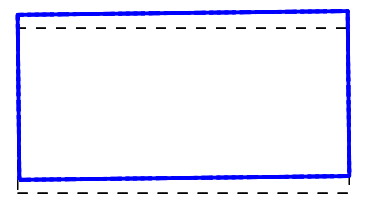

No Braces Floor

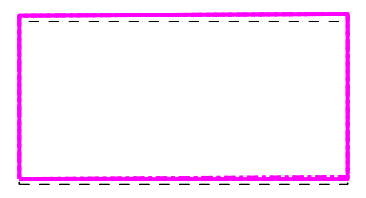

West Braces Only Floor

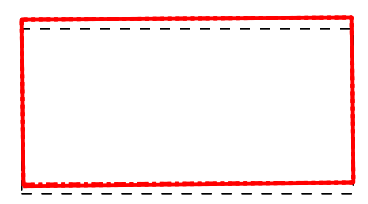

East Braces Only Floor

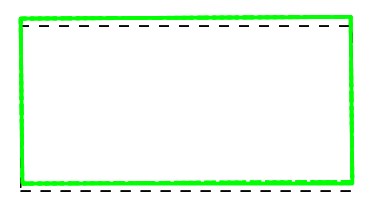

Undeformed Shape

$0.5 \mathrm{VPP}$

1.0 VPP ----- 1.5 VPP

$2.0 \mathrm{VPP}$

Figure 5-6: Normalized experimental modes

The voltage and amplitude of the shaker had little to no impact on the mode shapes.

Figure 5-6 show the normalized mode shapes for each voltage superimposed on one another. 
If examined closely, slight variances are observable. In summary, the amplitude of the shaker, and the corresponding force it outputs has no impact on the mode shapes for each brace configuration.

\subsubsection{Average Normalized Mode Shapes}

The mode shapes from the four different shaker amplitudes were averaged to give final experimental results. These results will be used as the experimental mode shapes. Because the mode shapes are independent of the shaker amplitude, averaging had little impact on the final results.

All Braces

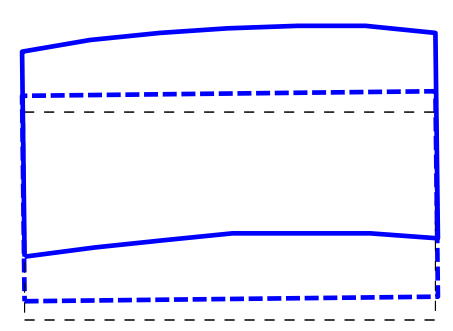

West Braces Only

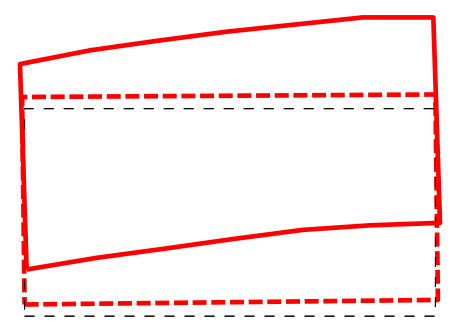

No Braces

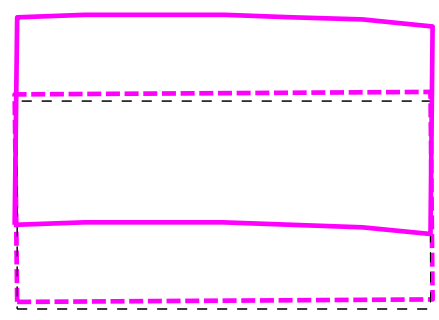

East Braces Only

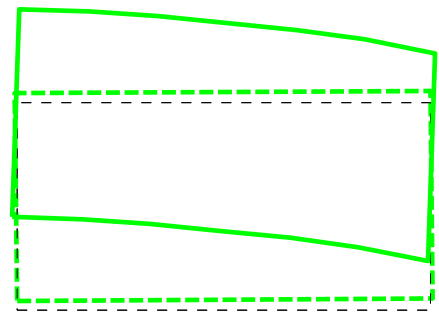

Figure 5-7: Averaged normalized experimental modes

The mode shapes for the different brace configurations behaved mostly as expected. The No Braces configuration behavior was slightly unexpected. Because of the additional flexibility of the pedestals on the East side of the Bridge House, it is expected that the deformations would be slighly larger on the East side. By examing the floor in the in the No Braces configuration, larger displacements are observed on the East side roof as expected. 
However, the additional stiffness of the non-structural elements on the East side decreases the respective roof displacements. The East side of the Bridge House has many additional elements that can contribute to the lateral stiffness as observed in Figure 5-8. These items are not present on the West side of the Bridge House.

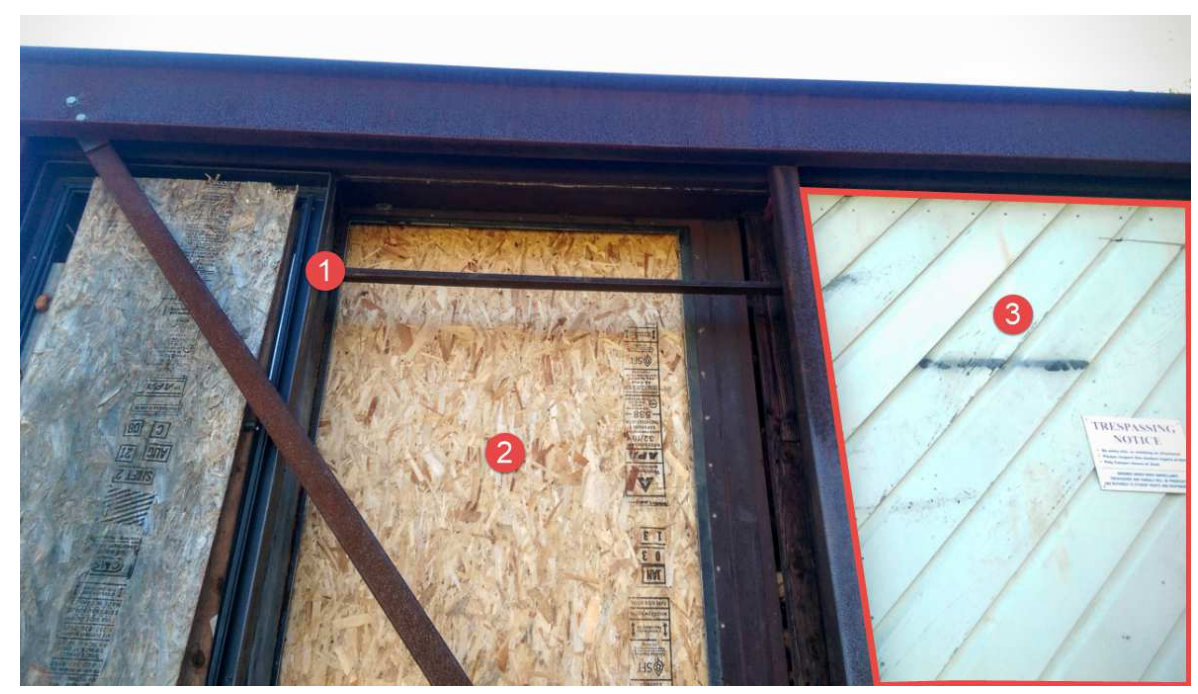

Figure 5-8: Examples of stiff non-structural elements

In Figure 5-8, highlighted item 1 is a steel mullion frame that occupies the majority of the frame, highlighted item 2 is a plywood sheet, and highlighted item 3 is timber planks. Typically, non-structural elements such as these in a building are far less stiff in respect to their lateral system than they are in the Bridge House. In the Bridge House, when braces are dissengaged, these items can contribute greatly to the lateral stiffness. These additional elements only impact the lateral stiffness when the braces are disengaged; the impact the additional elements have on the stiffness of the lateral system when the braces are engaged is negligible. This is not seen in the other brace configurations because the brace frames are far stiffer than the nonstructural elements, and therefore, the building behaves as expected.

In the West Braces Only and the East Braces Only brace configurations, the displacements are the greatest on the opposite sides. For example, if the West braces are engaged, the East side of the Bridge House is allowed to displace further. This behavior is 
expected. Interestingly, the behavior for all brace configurations is overwhelmingly translational, and all brace configurations look very similar. Out of all the brace configurations, the All Braces configuration also has larger relative floor displacements in comparision to the roof. This is explained by the fact that braces increase the story stiffness and reduce the roof's contribution to the final mode shape.

\subsubsection{Floor Behavior}

Superimposing the the floor behavior from all four brace configurations shows the floor behavior varies per brace configuration. By examining the behavior of just the floor, several observations can be made.

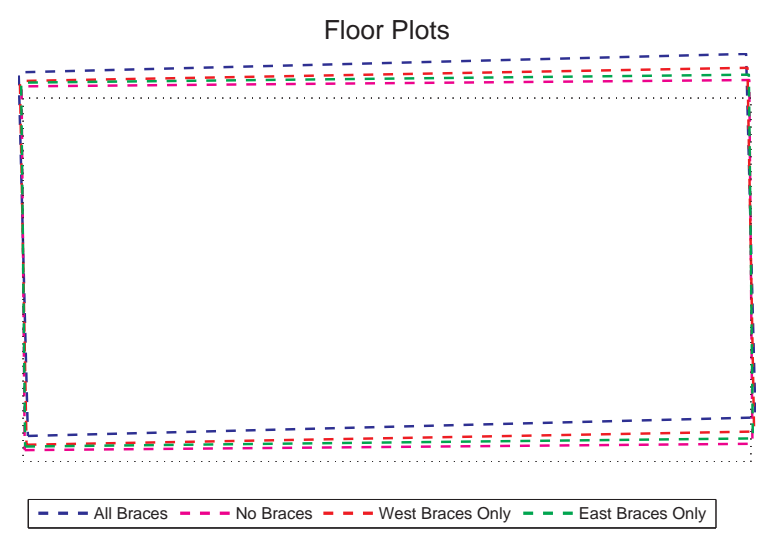

Figure 5-9: Superimposed floor averaged normalized mode shapes

As previously mentioned, the relative displacement in the All Braces configuration is greater than any other mode shape. Additionally, it appears the directions of the floor due to the brace configurations are the same; i.e., they have approximately the same amount of translation and rotation; the only difference is the scale. The difference is small, but it suggests that the floor behavior could be helpful in identifying brace configurations. 


\subsubsection{Roof Behavior}

Previous testing (Archer, 2014) indicated that the diaphragm may behave slightly irregular behavior. By subtracting the rigid body behavior from the accelerations, the diaphragm behavior can be isolated. The normalized roof behavior for each brace configuration is shown in Figure 5-10. Note that the figure is poorly scaled, the displacements would be several magnitudes less than length of the Bridge House.

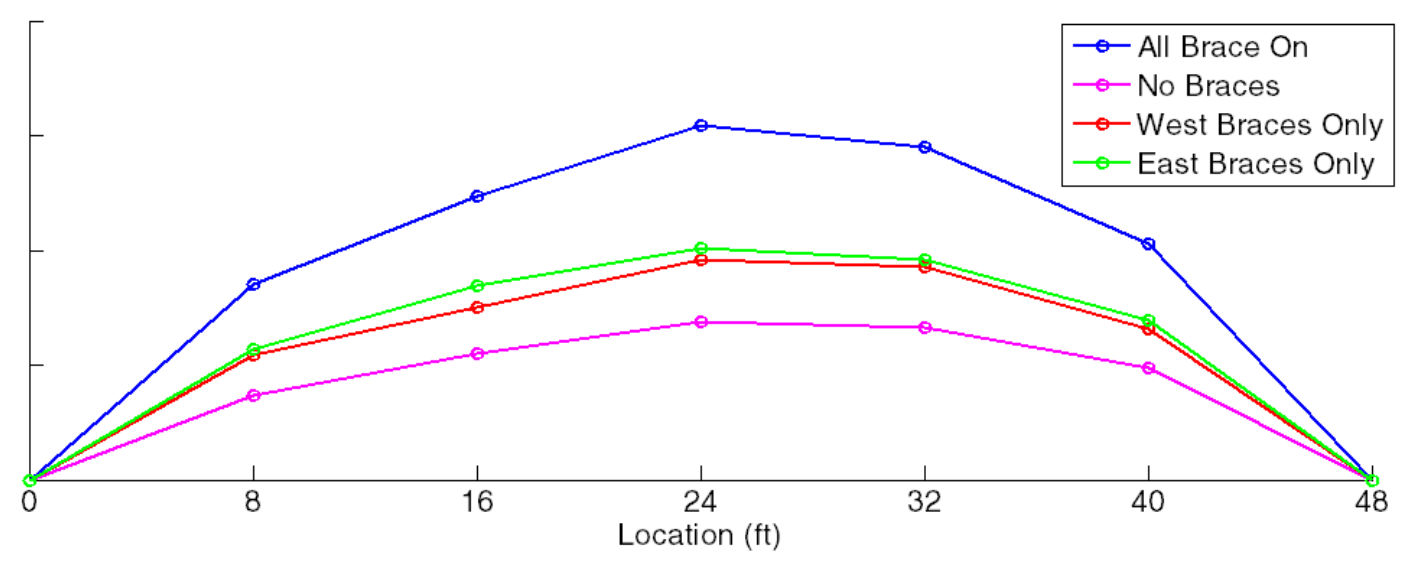

Figure 5-10: Isolated diaphragm behavior

For the West Braces Only and East Braces Only brace configurations, the displacements of the diaphragm are almost identical. This is expected, the diaphragm should deflect similarly when the stiffness of the lateral-force-resisting-elements are approximately the same. The stiffer the lateral-force-resisting-system, the larger the diaphragm displacements are. For every brace configuration, there is a slight tendency of larger displacements towards the East side of the structure. The cause of this is unknown. There is also a slight dip or decrease in all brace configurations around the 16 foot location.

\subsection{Substructure Stiffness}

The stiffness and condition of the substructure of the Bridge House is unknown. The steel frame rests upon concrete piers of various lengths. Each concrete pier has subterranean 
pedestals. The embedment depth, the height, and the stiffness of the pedestals are unknown.

The soil properties, along with the pedestal properties, significantly contribute to the deformations of the building and need to be accurately accounted for. System identification can be utilized to determine the stiffness of substructure elements in a building. The following derivation will outline how utilizing dynamics can determine the substructure stiffness. Stiffness of a linear elastic system is defined as the lateral force of an element divided by the corresponding displacement.

$$
k=\frac{f}{u}
$$

Using statics along with basic dynamic principles, the stiffness of each element of the substructure can be calculated from experimental data. The first step is to solve for inertia forces at each degree-of-freedom by utilizing Newton's Second Law:

$$
f_{i}=m_{i} a_{i}
$$

Where the acceleration, $a_{i}$, is the peak value recorded experimentally from each accelerometer and the mass, $m_{i}$, is the corresponding lumped mass as shown in Figure 5-11. Half of the story height of the structure is accounted for in the mass at each node.

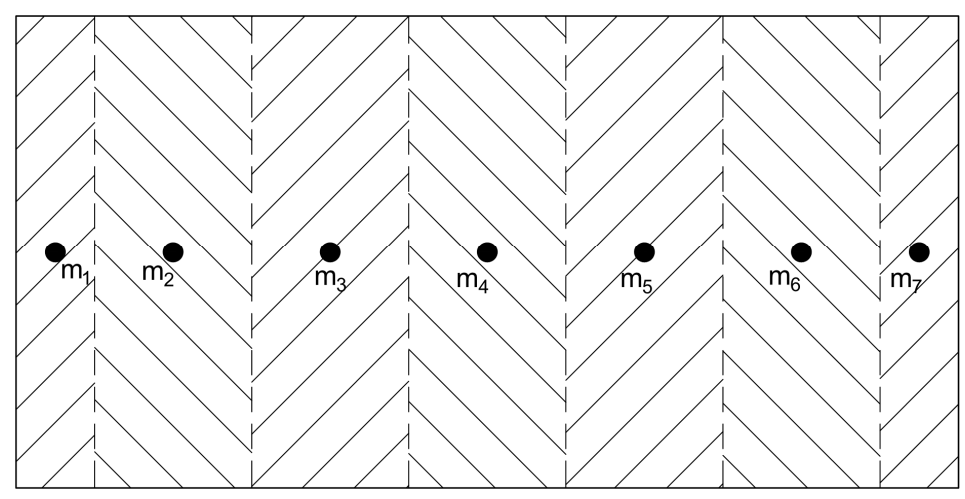

Figure 5-11: Lumped mass assumption for the roof and floor

Using Equation 5-2, the forces associate with each lumped mass, $m_{i}$, and the respective acceleration, $a_{i}$, along with the resultant forces are shown below. 


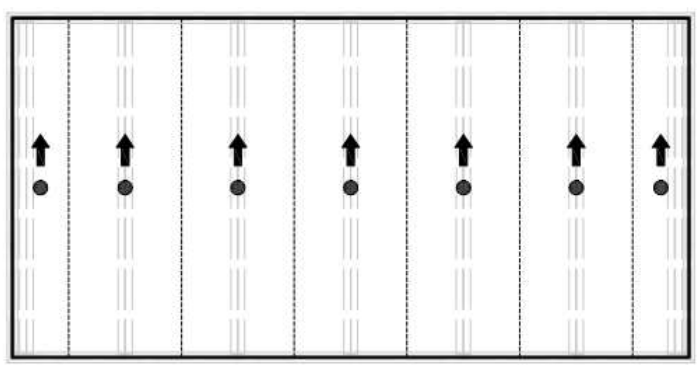

ROOF

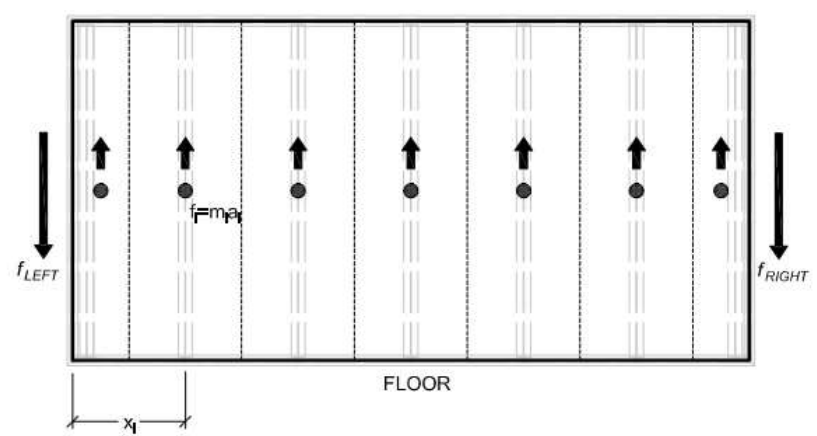

Figure 5-12: Force diagram for roof and the floor

When calculating the force transferred through the substructure, $F_{\text {LEFT }}$ and $F_{\text {RIGHT }}$ are the resisting forces of the substructure. The resultant forces are solved using statics. Summing moments around $f_{\text {LEFT }}$ and dividing by the distance of the entire building allows for the calculation of $f_{\mathrm{RIGHT}}$ as shown below. Fourteen terms are used to sum moments as it correlates to the fourteen degrees of freedom.

$$
f_{R I G H T}=\frac{\sum_{i=1}^{14} f_{i} x_{i}}{x_{R I G H T}}
$$

The distance from the East side to each degree-of-freedom is $x_{i}$ and the total length of the Bridge House is $x_{\text {Right }} . F_{L E F T}$ can be solved by summing forces in the y-direction.

$$
f_{L E F T}=\sum_{i=1}^{7} f_{i}-f_{R I G H T}
$$

To calculate the displacements, the solution describing a system subjected to a harmonic function at steady state can be utilized.

$$
u_{p}(t)=u_{\max } \sin (\omega t-\phi)
$$

Where $\omega$ is the frequency exciting the structure, $u_{\max }$ is the static displacement, and $\phi$ is the phase angle. The second derivative of Equation 5-5 with respect to time yields the accelerations. 


$$
\ddot{u}_{p}(t)=-w^{2} u_{\max } \sin (\omega t-\phi)
$$

Equation 5-6

Substituting Equation 5-5 into Equation 5-6 yields:

$$
\ddot{u}_{p}(t)=-w^{2} u_{p}(t)
$$

Equation 5-7

Re-arranging gives:

$$
u_{p}(t)=\frac{\ddot{u}_{p}(t)}{-w^{2}}
$$

Because the concern is the maximum values at the natural frequency, the negative sign will be removed, the frequency component shall be replaced with the natural frequency, and the time dependency of the function is irrelevant and will be removed:

$$
u_{p}=\frac{\ddot{u}_{p}}{w_{n}{ }^{2}}
$$

Equation 5-9

Equation 5-9 establishes a relationship between the displacements at the accelerometer locations and the respective accelerations. Equation 5-9 can be substituted into Equation 5-1:

$$
k=\frac{f}{\frac{\ddot{u}_{p}}{w_{n}{ }^{2}}}
$$

Where $f$ is force traveling through the substructure (either $f_{\text {LEFT }}$ or $f_{R I G H T}$ ), $\ddot{u}_{p}$ is the acceleration at the respective location, and $w_{n}$ is the fundamental frequency of the first mode being tested. Using dynamics and equilibrium, the stiffness of the substructure has been identified. Further assumptions are still necessary to adapt the proof to the Bridge House. There are a total of four piers at the Bridge House, two per gridline. $F_{\text {LEFT }}$ and $F_{\text {RIGHT }}$ correspond to the forces that occur at both piers within lateral system. Every pier has a different length and different embedment into the soil. 
Instead of modeling the concrete piers and the soil, all substructure behavior will be modeled with one degree-of-freedom springs. The stiffness calculated from the above derivation will be assumed to be evenly distributed between both piers. This assumption is only valid as the behavior is overwhelmingly purely translational. Out-of-plane vertical behavior perpendicular to the face of the floor was not examined for this research, and a roller support is used for model stability. A simplification of substructure behavior is shown in Figure 5-13.
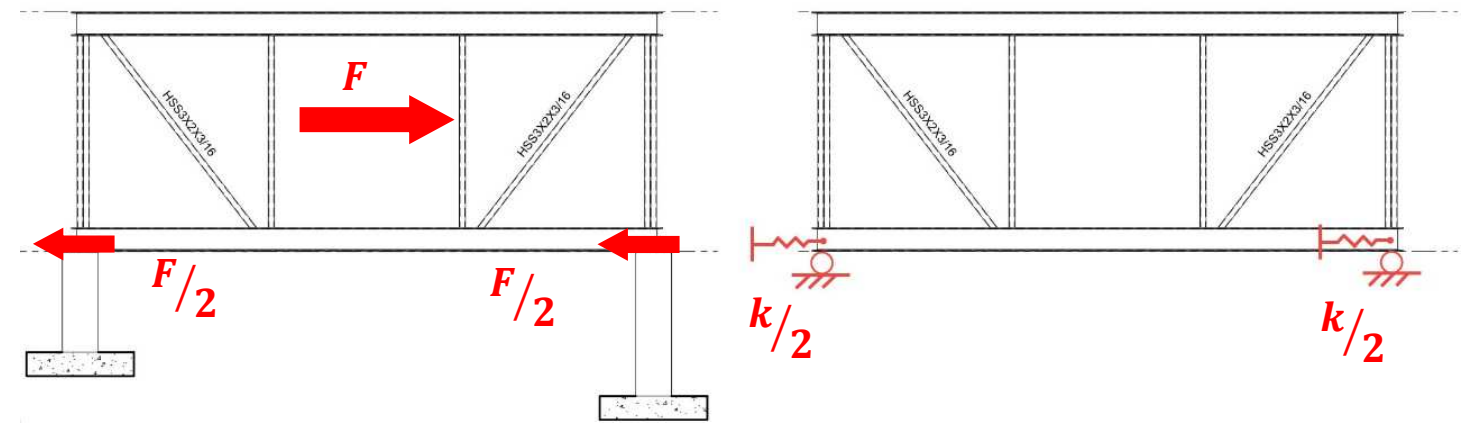

Figure 5-13: Suspected as built (left), Theoretical model (right)

Figure 5-13 shows rollers in the vertical direction. The influence of the vertical behavior is irrelevant to this research; hence, rollers were used for model stability. A plan view of the theorized substructure springs are shown in Figure 5-14.

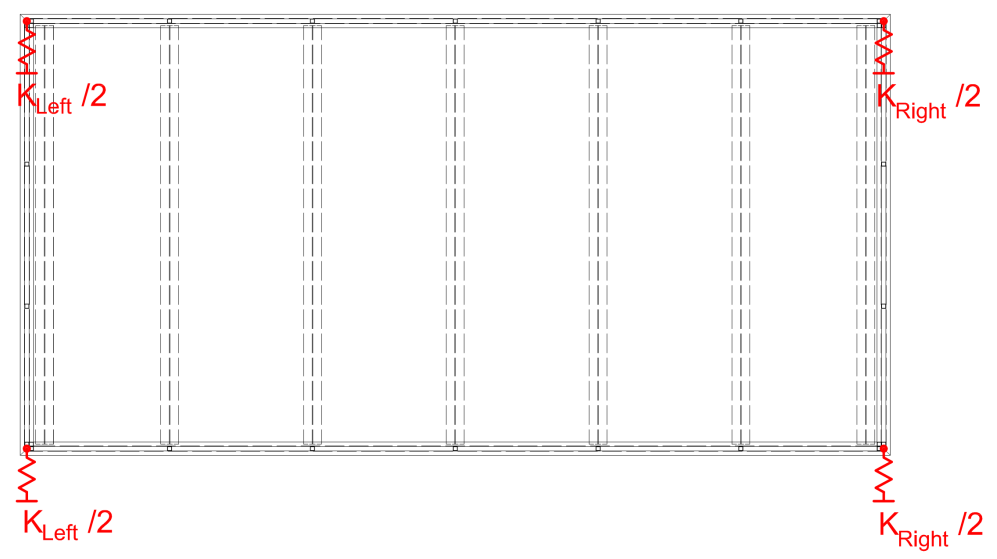

Figure 5-14: Calculated substructure springs

One degree-of-freedom springs with the same stiffness are used perpendicular to the direction tested. No data was tested in the perpendicular direction, but because the pedestals 
are symmetrical, it can be surmised that all substructure behavior is identical in both directions.

This assumption is shown in Figure 5-15.

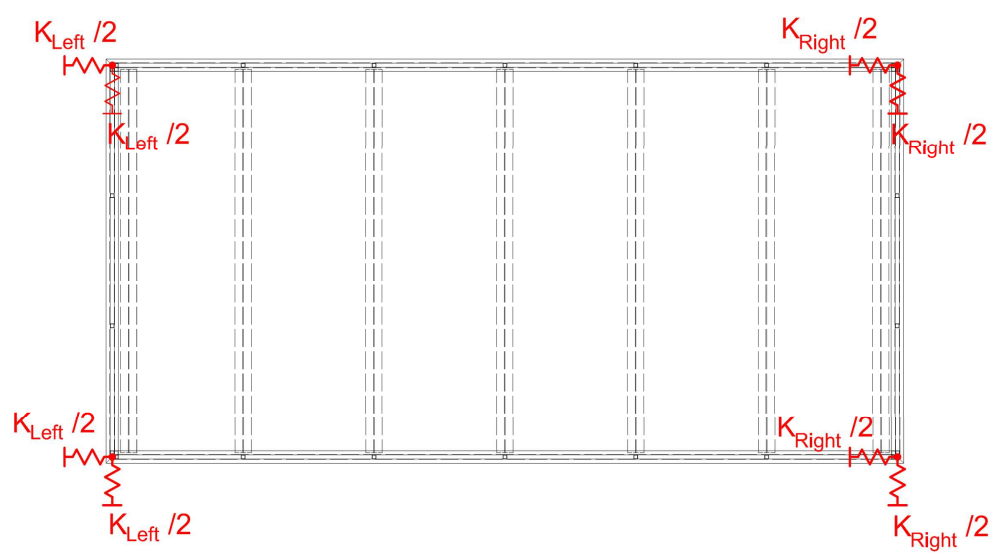

Figure 5-15: Finalized substructure springs

A plot of the results of the substructure stiffness with respect to the forcing amplitude is shown below.

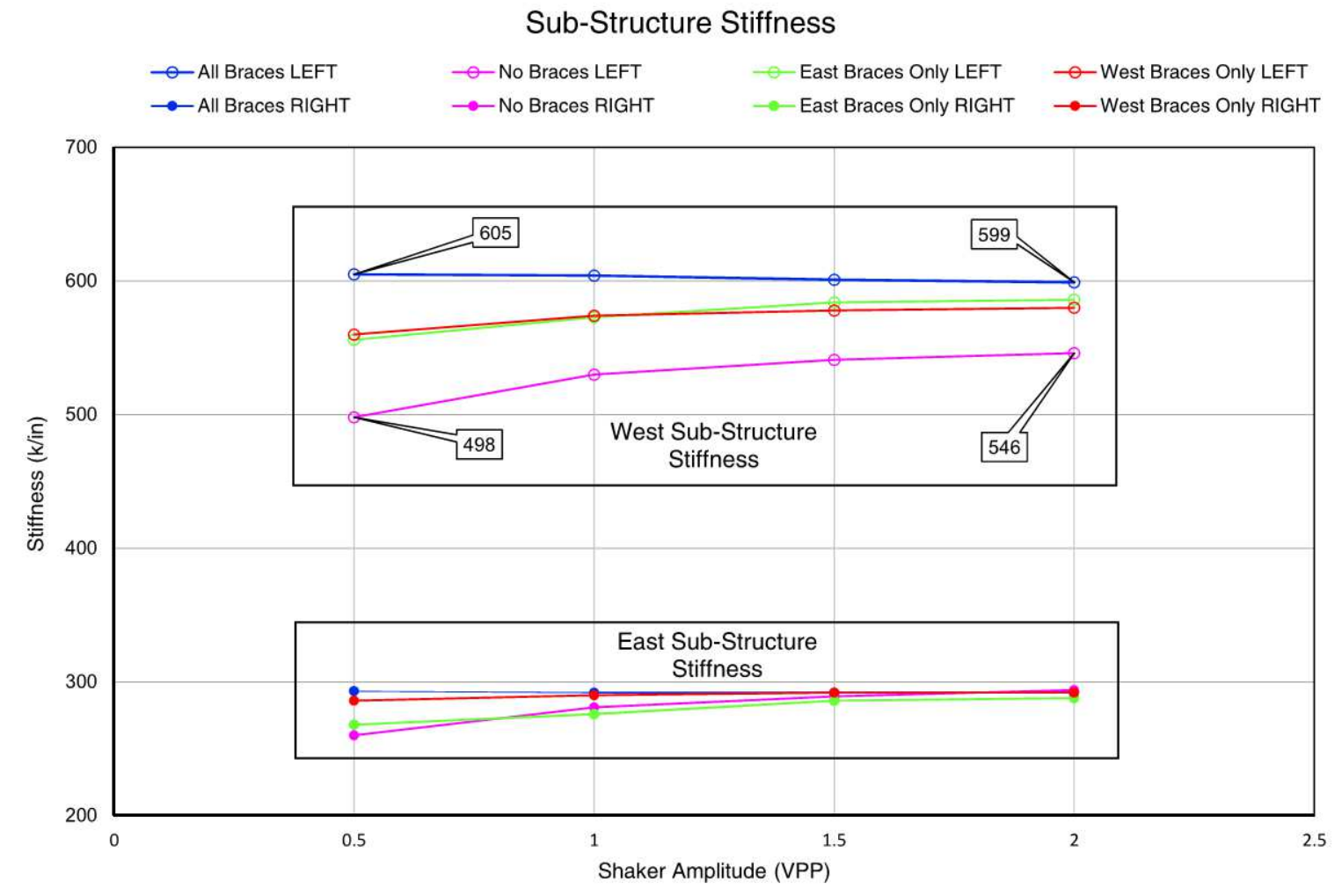

Figure 5-16: Experimental substructure stiffness 
As expected, the West side of the Bridge House had much stiffer substructure behavior than the East side. This is contributed to the exposed and flexible piers on the East side of the structure. Additionally, there appears to be a non-linearity with respect to the amplitude of the shaker and brace configurations. At lower amplitudes, there was a greater variance of the substructure stiffness between brace configurations, especially on the West side of the structure. As amplitude of the shaker increased, the stiffness values between brace configurations converge. It is not known if the original differences in calculated stiffness is due to frequency dependent soil properties or if the different mode shapes yield different values. Even though there is an apparent variance on the West side of the Bridge House, the difference between the brace configurations is approximately 17 percent for 0.5 VPP and 10 percent for 2.0 VPP. Overall, the variance in substructure behavior is small.

\subsection{Lateral Force Resisting System Stiffness}

Unlike the substructure, the behavior of the lateral system on the Bridge House is well defined. The lateral system is a hybrid of a Vierendeel trusses and a brace frame configuration on the West and East exterior faces, and "moment frames" on the interior gridlines. Both of these can accurately be modeled within a finite element program. Regardless, stiffness identification of the brace line can still be used to verify a theoretical model. To identify the lateral stiffness, the same theory can be used that determined the substructure stiffness. A revised free body diagram is shown below. 

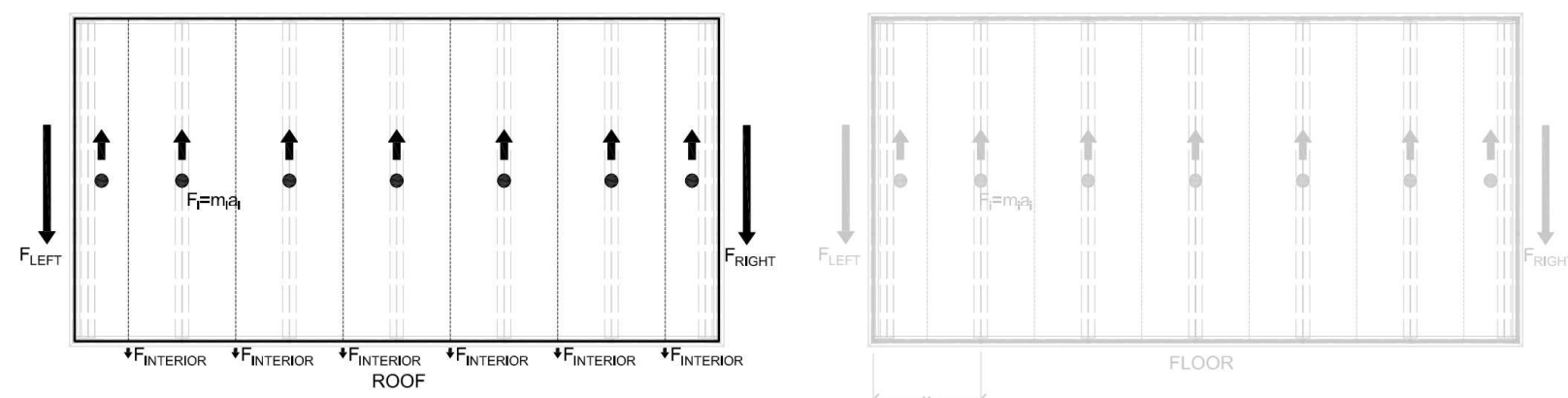

Figure 5-17: Free body diagram of the roof

The floor free body diagram has been greyed out to indicate it is not needed for this analysis. The resistance of the interior moment frames has been added as well. When all the braces are engaged, it is assumed that the perimeter lateral system is much stiffer than the interior moment frames. This assumption can be made for the following reasons. First, the built-up columns on the Vierndeel truss have a higher moment of inertia and are much stiffer than the interior moment frames. Second, the perimeter lateral system have beams with onethird the length of the interior moment frames. Third, the base of the perimeter lateral system is fixed against rotation whereas the interior moment frame bases are free to rotate. And fourth, the perimeter lateral system has braces. For these reason, the stiffness contribution of the interior moment frames is neglected. The corresponding forces of the perimeter lateral are named $F_{\mathrm{RIGHT}}$ and $\mathrm{F}_{\mathrm{LEFT}}$; a revised free body diagram is shown below.
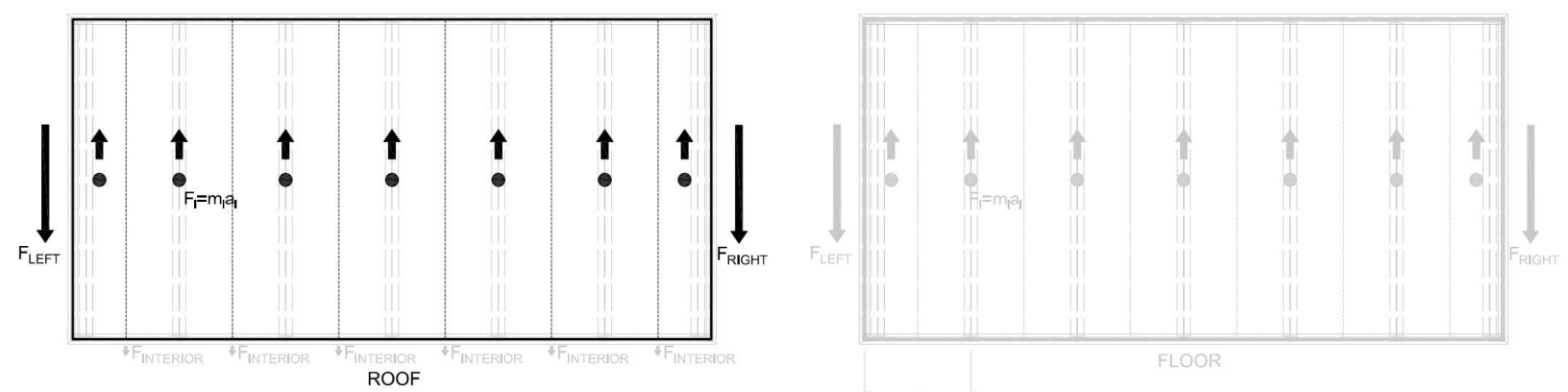

Figure 5-18: Revised free body diagram of the roof 
Using the same principles outlined in the substructure stiffness identification, the stiffness of the lateral-force-resisting elements can be identified. Only the All Brace On system was tested as it was assumed that exterior brace frames were much stiffer than the interior moment frames. Once the exterior braces were disengaged, this assumption no long holds valid. The calculated stiffness of the lateral force resisting system was highly reproducible for trials with different shaker amplitudes. See Table 5-3 below for the results.

Table 5-3: LFRS stiffness for All Braces On

\begin{tabular}{|c|c|c|}
\hline Trial & $\begin{array}{c}\text { West LFRS } \\
\text { (kip/in) }\end{array}$ & $\begin{array}{c}\text { East LFRS } \\
\text { (kip/in) }\end{array}$ \\
\hline 0.5 VPP & 91.7 & 100.59 \\
\hline 1.0 VPP & 91.72 & 100.07 \\
\hline 1.5 VPP & 91.67 & 99.80 \\
\hline 2.0 VPP & 91.65 & 99.81 \\
\hline
\end{tabular}

The stiffness of the East LFRS is slightly higher, as expected. This is likely due to the additional non-structural elements on the East side. Additionally, the stiffness did not change with regards to the amplitude of the shaker. This suggests that other non-structural items are not being engaged as displacements increased; the stiffness of the lateral force resisting system is much greater than the non-structural when the braces are engaged. 


\subsection{ANALYTICAL MODELS}

\subsection{Background}

Analytical models were made using SAP2000 (CSI, 2013). All major structural elements were defined as they were surveyed in the field. Because of the design of the Bridge House, assumptions were necessary to accurately model the building. The results from the system identification for the roof and soil springs were implemented into the model.

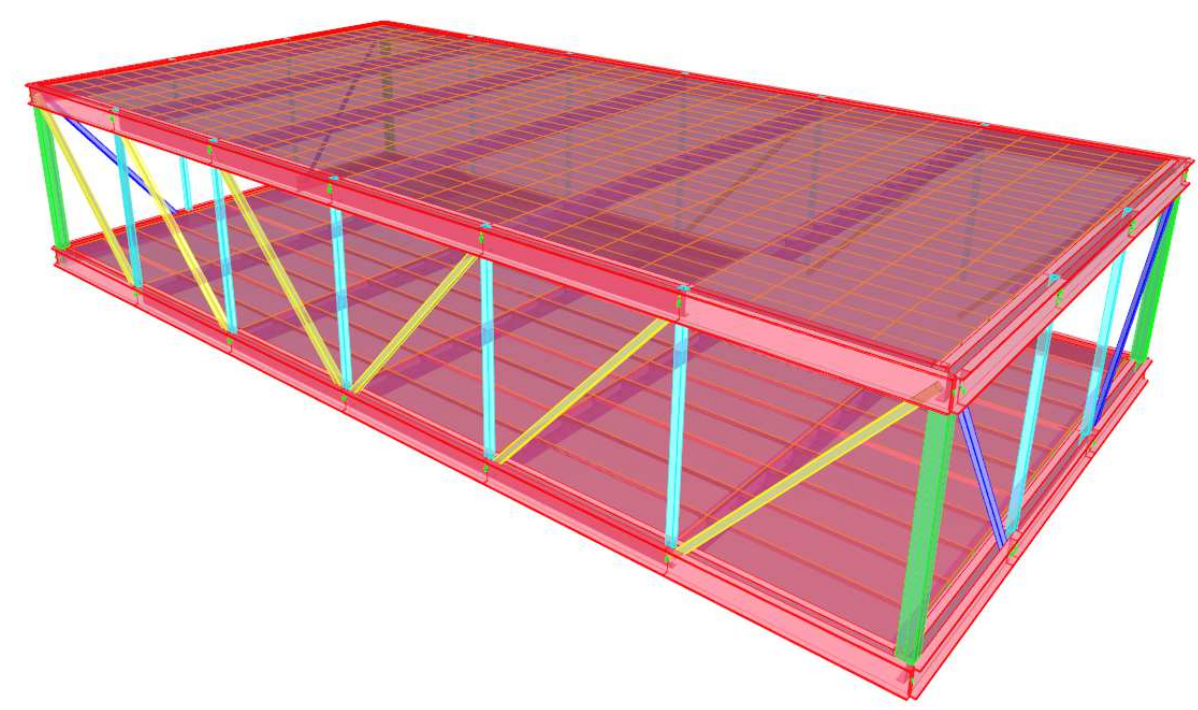

Figure 6-1: Analytical model

\subsection{Built-Up Sections}

Built-up columns and sections are found at several locations throughout the Bridge House. Because of the various as-built conditions, each section was addressed independently. Many connections had several members joining with redundant welding.

The corner columns of the Bridge House have ten members that frame into the start and end nodes. No intermediate welds were found between the HSS columns. To model these connections, each frame member was according to the actual geometry at the Bridge House. 
To model welds, it was strived to use a minimum number of rigid links were used that would capture the dozens of welds found at the connections at the Bridge House.
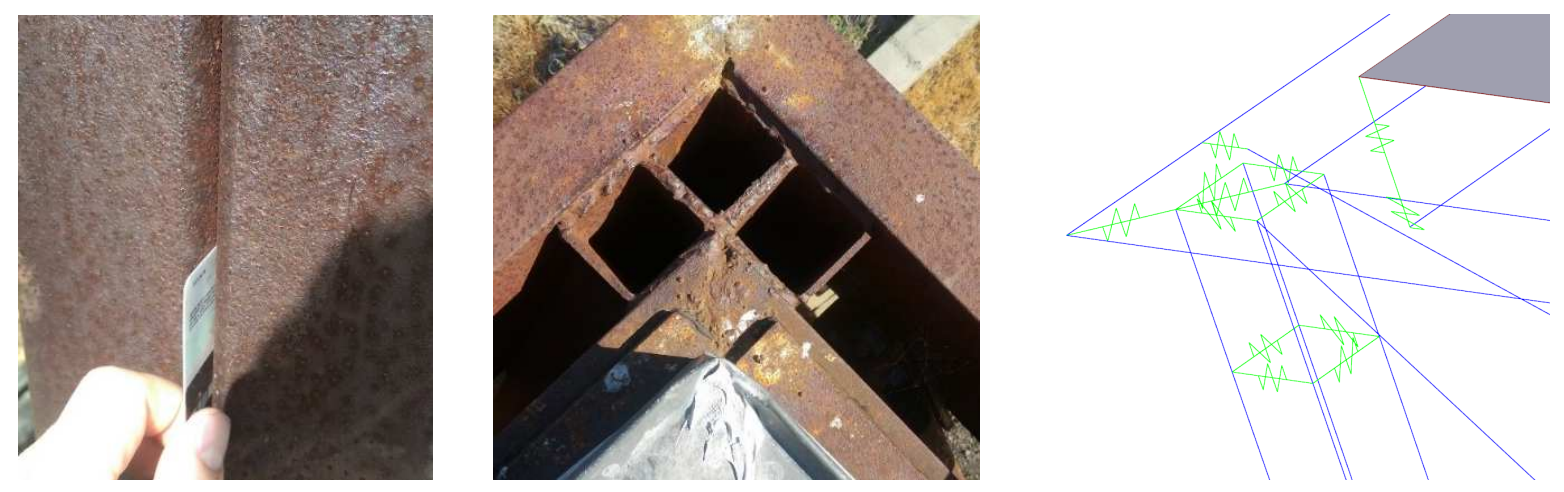

Figure 6-2: Corner detail

Interior columns also were discovered to be made up of multiple shapes. During construction, additional steel sections were used to make mullions for the glass panels. These mullions were irregularly spot welded to the HSS $3 \times 3 \times 1 / 4$ column at various intervals and lengths.
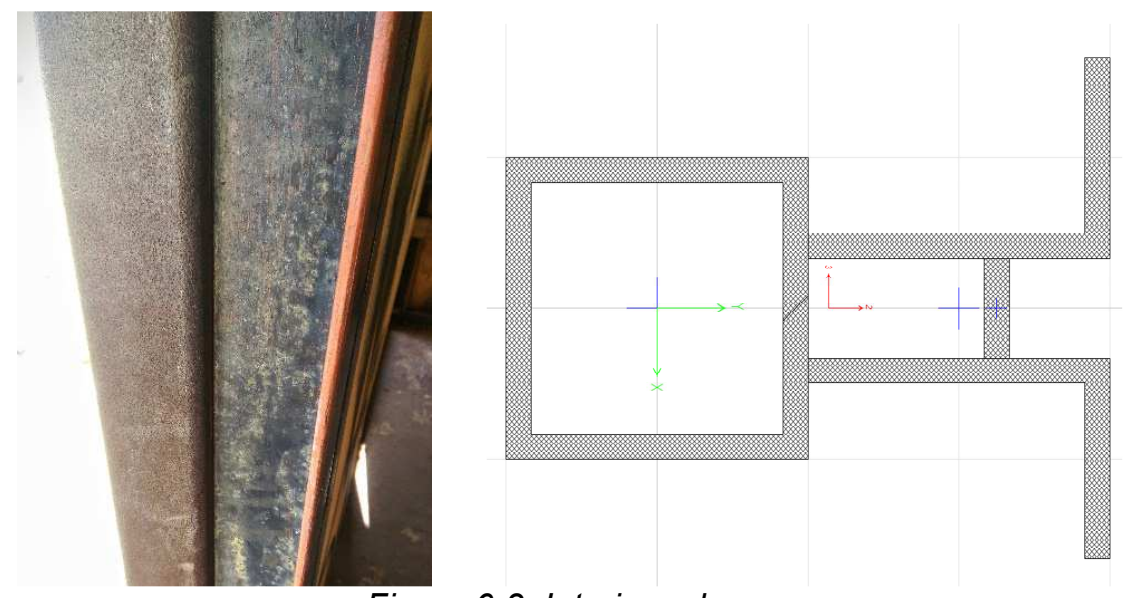

Figure 6-3: Interior column

The moment of inertia of the built-up section is $22 \mathrm{in}^{4}$ while the moment of inertia of an HSS $3 \times 3 \times 1 / 4$ is $3.02 \mathrm{in}^{4}$. Because of the inconsistent and intermediate welds, the section cannot be classified as a built-up section. To estimate this intermediate behavior, an effective section modulus of $12.08 \mathrm{in}^{4}$ was chosen. This is four times larger than the regular section modulus of the HSS $3 \times 3 \times 1 / 4$. This modeling assumption was based off engineering judgement. 
Because these columns do not greatly contribute to the lateral stiffness, any reasonable approximation could be used.

\subsection{Joints}

For low-level forced vibration testing, all connections are modeled as fixed. Low-level forced vibration testing puts minimal loading through the structure. Connections that are typically modeled as pinned during analysis and design, are fixed for the small loads seen during forced vibration testing. Figure $6-4$ below shows a shear tab that would typically be modeled as pinned for analysis but would be able to fully resist any forces that are seen during forced vibration testing.

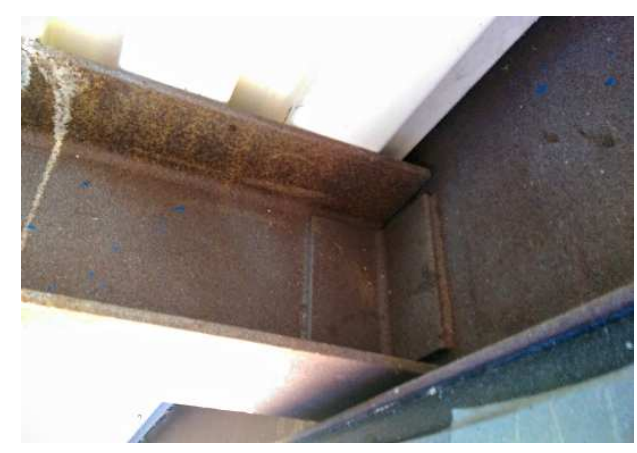

Figure 6-4: Shear tab examples

\subsection{Substructure Springs}

All substructure behavior was modeled with individual springs with results found from system identification. This methodology is outlined in section 5.6. The results from the substructure system identification are shown below. 


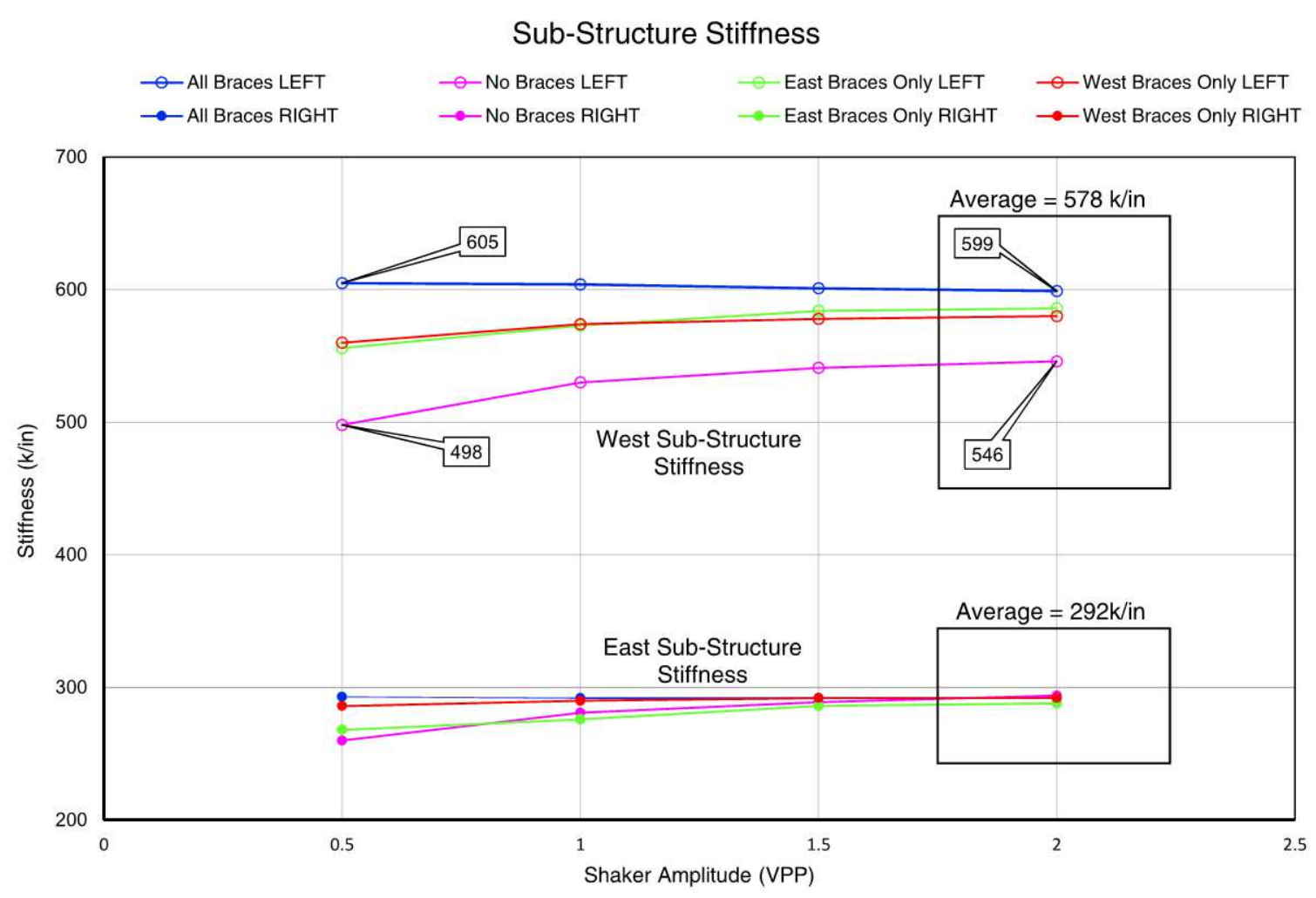

Figure 6-5: Averaged experimental substructure stiffness

Figure 6-5 shows the average values for each side of the Bridge House. The largest tested shaker amplitude was selected as it appears there is a convergence of values for a larger shaker input. Even with one brace configuration, a convergence would still be observed. Additionally, values were averaged between the brace configurations. In application, after an earthquake, averaging would not be possible as there would not be multiple brace configurations to test. For this research, it is acceptable as the difference between the stiffness values is negligibly small. This assumption will be examined in later section. These stiffness values were divided in half (one for each pier) and springs were applied to the model. 


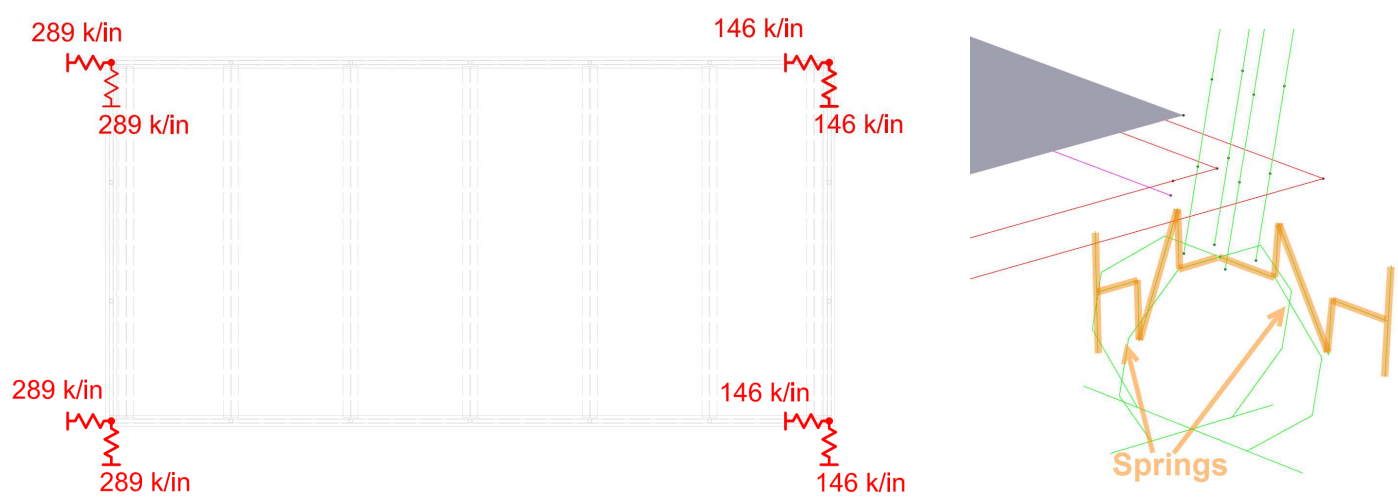

Figure 6-6: Applied substructure springs

As discussed in section 5.6, springs horizontal to the tested direction were also applied for stability. It was assumed that for every pier, the horizontal behavior was identical to the vertical behavior.

\subsection{Single Frame Model}

A model of a single exterior frame line was made to examine the stiffness of the East and West faces. A unit load was applied deflections were measured. From here, the lateral stiffness can be calculated.

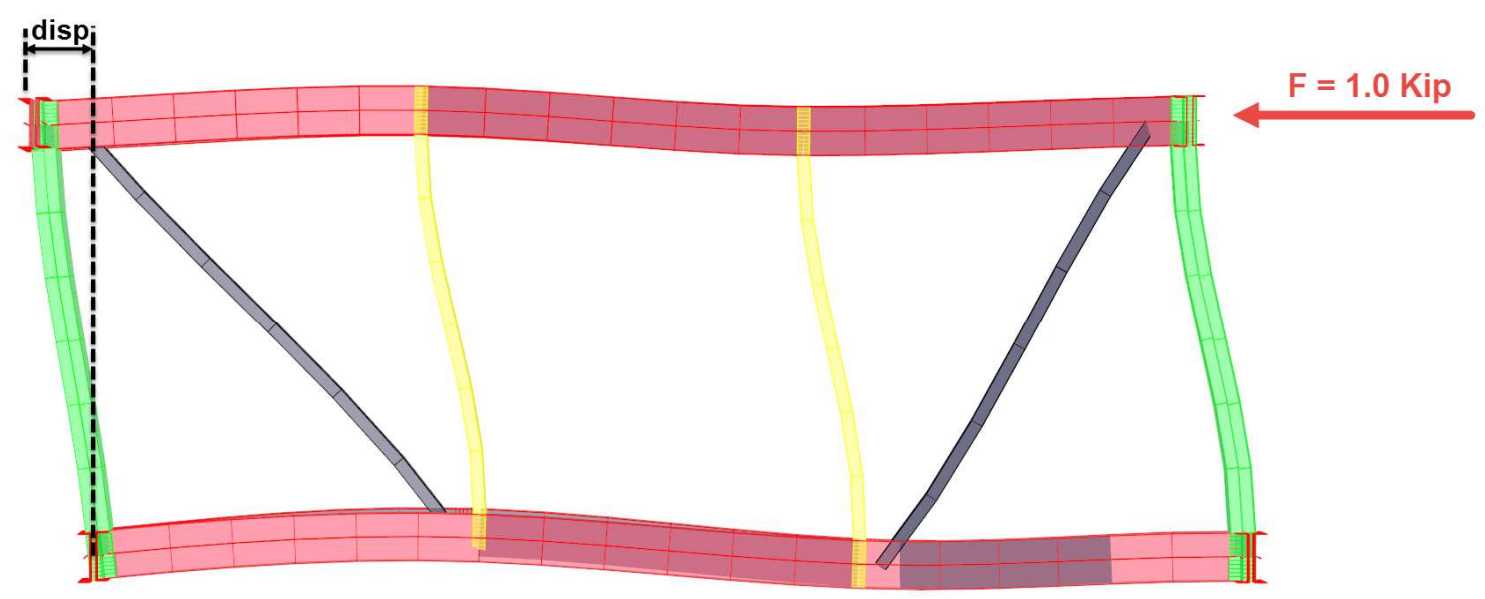

Figure 6-7: Single frame model

The theoretical results, along with the experimental results of the All Braces On configuration, are shown in Table 6-1. 
Table 6-1: Model LFRS Stiffness

\begin{tabular}{|c|c|c|}
\hline Brace Configuration & Model Stiffness (kip/in) & $\begin{array}{c}\text { Experimental Stiffness } \\
\text { (kip/in) }\end{array}$ \\
\hline All Brace On & 82.3 & $91-101^{*}$ \\
\hline 1 Brace & 36.4 & N/A \\
\hline No Braces & 11.8 & N/A \\
\hline \multicolumn{2}{|c|}{ *101 kip/in for the East LFRS, 91 kip/in for the West LFRS } \\
\hline
\end{tabular}

The only values to compare to the experimental values are the All Braces On. The model yields slightly lower values. This is expected as none of the non-structural elements that contribute to the stiffness were modeled. Because of the robustness of these elements, the approximately 10 to 20 percent difference is reasonable. These results help establish the validity of the model. Additionally, there is significant decrease in stiffness when braces are removed. When removing just one brace, the stiffness decreases by $45.9 \mathrm{kip} / \mathrm{in}$, more than half. When both braces are removed, an additional stiffness loss of $24.6 \mathrm{kip} / \mathrm{in}$ is recorded. Clearly, the behavior of the frame line behaves as a combination lateral system. Previously, this frame line was described as a Vierendeel truss with brace frame infill. By examining the results of this stiffness study, this classification is acceptable.

\subsection{Roof Properties}

Shell elements were chosen model the roof deck. To identify the equivalent thickness and stiffness of the roof diaphragm, an iterative method was established based upon a finite element model. To isolate the diaphragm behavior, it was assumed that the accelerations can be broken into two categories, diaphragm bending and rigid body behavior. A chord was drawn between the two edges of the roof to indicate the rigid body behavior of the roof. A typical deflected shape of the Bridge House, along with a chord between the two edges of the roof, is shown below. 

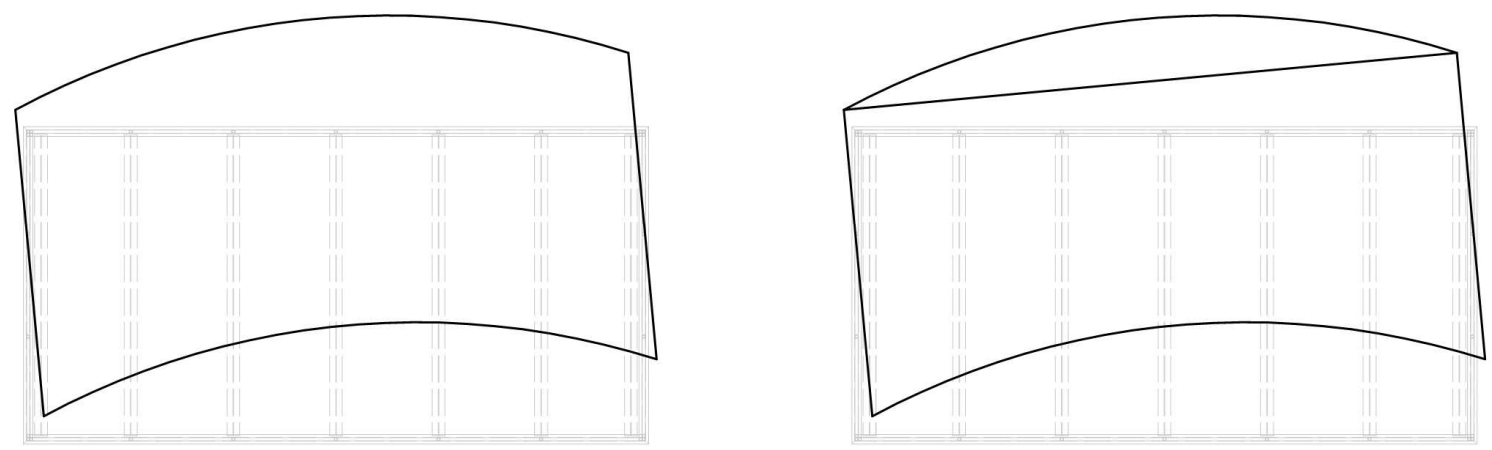

Figure 6-8: Typical roof deflection

It is assumed that the area between the chord and the deflected roof shape is due to the diaphragm, and the remained of the area is due to rigid body behavior. After subtracting the rigid body behavior of the roof and the floor from the total deflections, the remaining deflection is the deflection due to the diaphragm only.

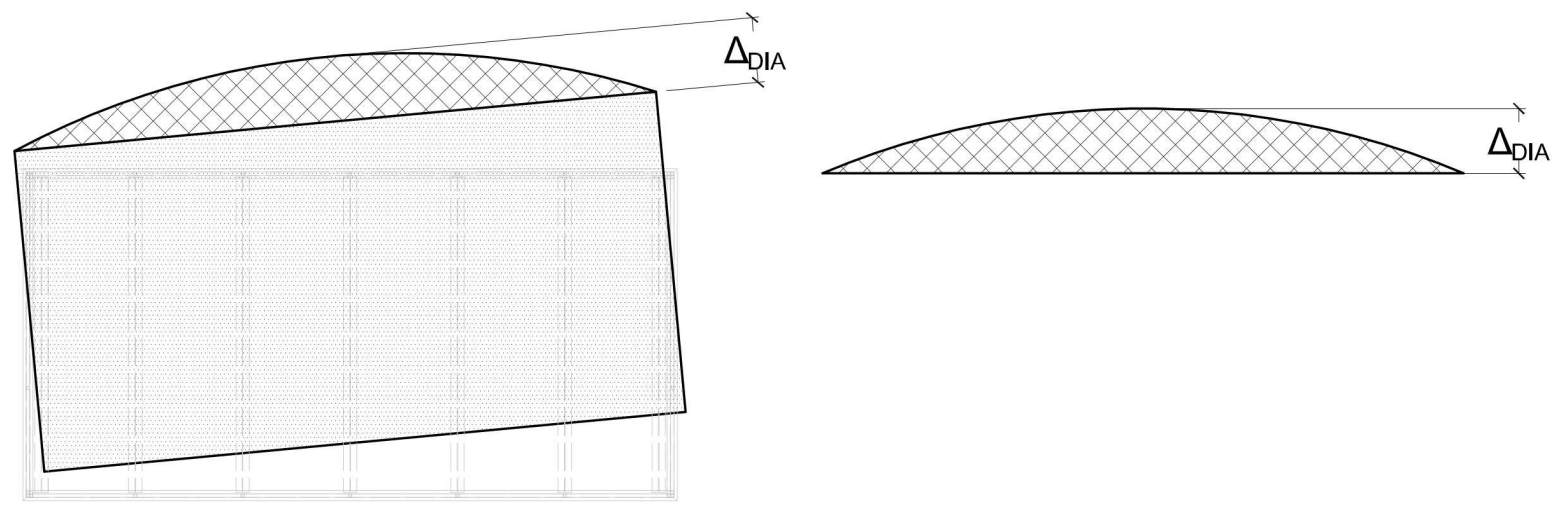

Figure 6-9: Components of roof deflection

The light dots in Figure 6-9 shows the rigid body behavior, the large hatch shows the deflections of the diaphragm. The isolated displacements of to the diaphragm can be determined. Using these results, an iterative approach was taken to determine an equivalent shell thickness in the model.

As outlined in the previous sections, the forces at the roof are well known due to Newton's Second Law. Instead of using point loads as was used for the other stiffness 
identification methods, the loads will be applied as a distributed load. The sum of all the forces at each lumped mass will be divided by the length (48 feet) to get an equivalent distributed load.

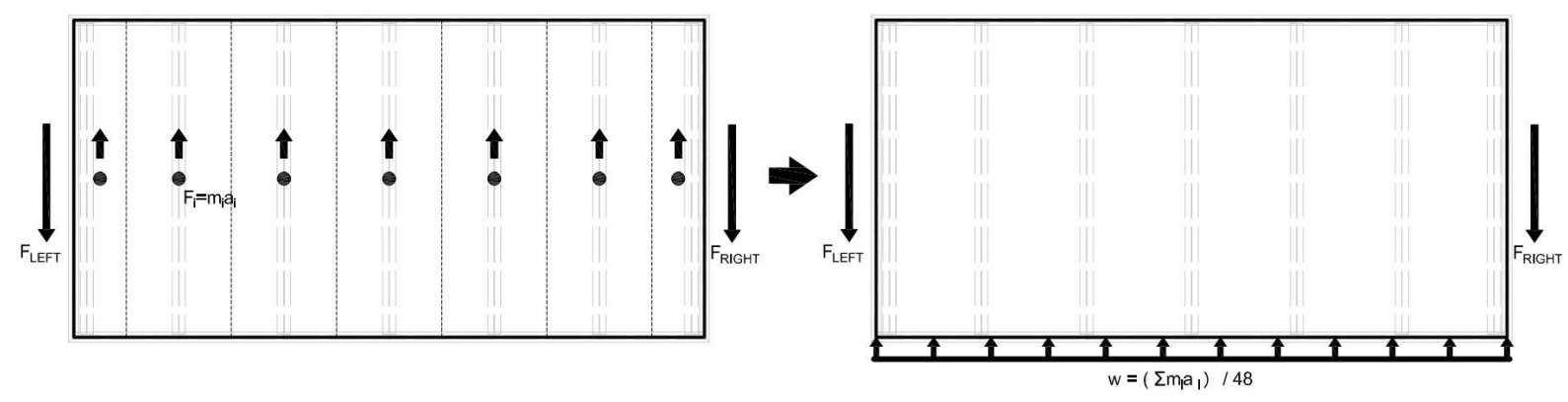

Figure 6-10: Distributed load assumption for roof stiffness identification

A detailed finite element model was made of the roof. The behavior of a squat roof diaphragm closely resembles that of a deep beam. The majority of displacements are due to shear deformations instead of bending. A free-body-diagram and deflected shape are shown below in Figure 6-11.
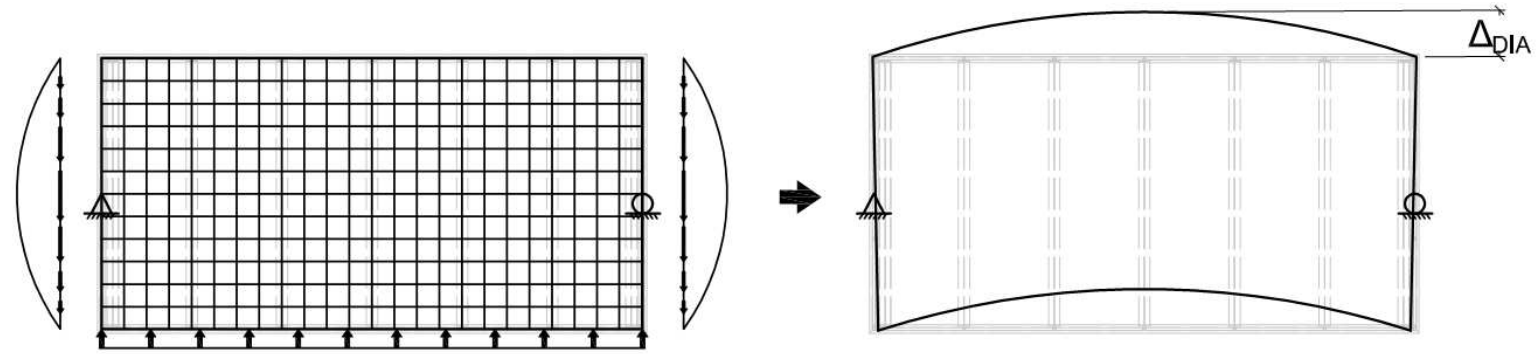

Figure 6-11: FBD of roof model

Because the roof was treated as a squat beam, it was modeled as simply supported. By adjusting the thickness of the shell elements, the displacements can be recorded for the loads based on the relevant brace configuration. Once displacements are found that closely matches the peak displacements at the midspan from the experimental results, the corresponding shell thickness is recorded and applied to the real model. For the Bridge House, this must be done for each brace configurations to ensure accuracy as each configuration has different applied 
forces and displacements. Each brace configuration should yield consistent results for the chosen thickness in order to be vetted as a reasonable estimate.

The model of the roof included membrane elements to model the roof decking and insulation, as well as frame elements to capture chords, collectors, and beams. All out-of-plane behavior was restricted with roller constraints. The stiffness of columns resisting the lateral forces was accounted for by implementing in-plane springs. An image of the SAP2000 model is shown in Figure 6-12.

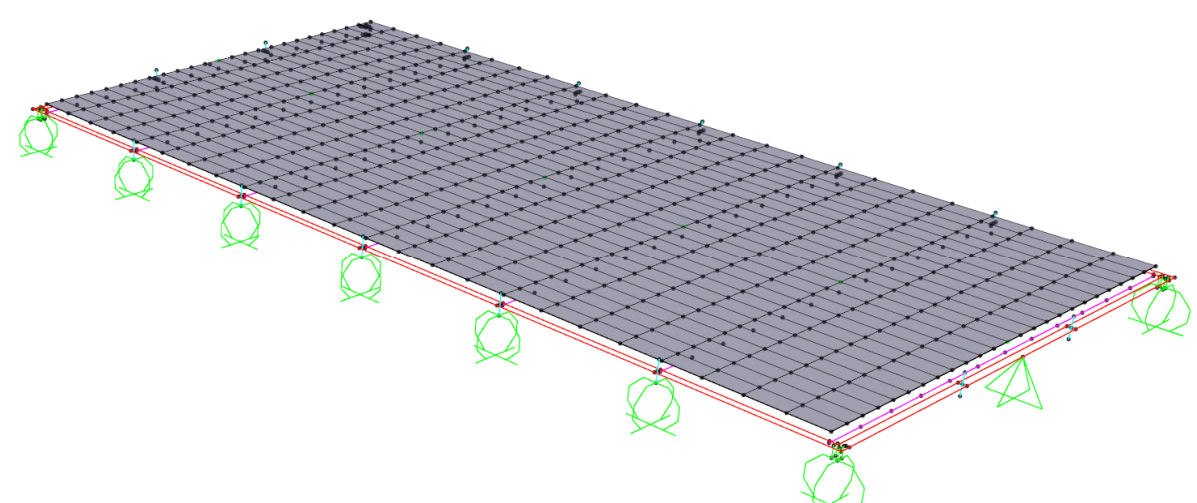

Figure 6-12: Roof model

It was found that a roof shell thickness of 0.0022 inches made from steel yielded the best results. The results of the chosen thickness are shown below in Table 6-2.

Table 6-2: Roof thickness study

\begin{tabular}{|c|c|c|c|}
\hline $\begin{array}{c}\text { Brace } \\
\text { Configuration }\end{array}$ & Target Disp. (in) & Model Disp. (in) & Percent Off \\
\hline All Braces On & 0.000863 & 0.000764 & $11.4 \%$ \\
\hline No Braces & 0.000498 & 0.000554 & $-11.3 \%$ \\
\hline
\end{tabular}

The two brace configurations tested represented the bounds of the roof diaphragm flexibility. The All Braces On configuration yielded results that were approximately 11 percent too stiff, and the No Braces configuration yielded results that were 11 percent too flexible. 


\subsection{METHODS OF DAMAGE DETECTION}

\subsection{Scope of Damage Detection}

In vetting the ability to detect and locate damage of a building, a series of studies will be conducted that tests the model robustness and the data acquisition process. These studies will have slight changes to either the model or data acquisition process. Several techniques will be used to evaluate how these changes impacted the ability to locate damage.

\subsection{Parametric Studies and Definitions}

Several definitions will be sustained throughout the damage detection sections. These definitions are listed below.

- Reference Baseline Model: A reference model created matching the geometric and section properties of the as-built conditions. Unknown elements, such as the roof and the substructure, were modeled using system identification. Typically abbreviated to Baseline Model to save space in figures.

- Parametric Theoretical Model: A model geometrically similar to the Baseline Model, with slight variations. Used to examine how differences in modeling assumptions impact final results. Frequently paired with an adjective to help identify the specific parametric study.

- Experimental Results: The mode shapes found from final experimental testing. 
Three groups of studies will be done to investigate the damage detection. The studies are outlined in Table $7-1$.

Table 7-1: Parametric study table

\begin{tabular}{|c|c|c|}
\hline Study & Question & Comparison \\
\hline Baseline & $\begin{array}{c}\text { Is damage detection through } \\
\text { FVT possible? }\end{array}$ & $\begin{array}{c}\text { Reference Baseline Model vs } \\
\text { Experimental Results }\end{array}$ \\
\hline $\begin{array}{c}\text { Parametric Model } \\
\text { Changes }\end{array}$ & $\begin{array}{c}\text { How robust of a model is } \\
\text { needed? }\end{array}$ & $\begin{array}{c}\text { Parametric Theoretical Model vs } \\
\text { Reference Baseline Model }\end{array}$ \\
\hline $\begin{array}{c}\text { Parametric } \\
\text { Procedural Changes }\end{array}$ & How much data is needed? & $\begin{array}{c}\text { Reference Baseline Model vs } \\
\text { Experimental Results (Revised DOF) }\end{array}$ \\
\hline
\end{tabular}

The first study will compare experimental results to the Baseline Model. This will establish if damage detection is possible using forced vibration testing. The second study will compare the Baseline Model to the Theoretical Model to help understand the impact of model accuracy. The third study will examine the consequences of changing the experimental degrees-of-freedom in terms of damage detection. The changed degrees-of-freedom were systematically chosen to replicate a scenario where a limited amount of accelerometers are available. For each study, the mass matrix must be reformulated. System identification, however, was not re-established with the new DOF.

\subsection{Assessment Methods}

\subsubsection{Frequency Analysis}

When applicable, frequency analysis will be examined. Previous research has shown that comparing experimental natural frequencies to theoretical natural frequencies has limitations (Archer, 2014). Regardless, theoretical frequencies must be reasonable and approximate or else the validity of the model needs to be questioned. It will be shown that a frequency analysis can be used as model verification to set upper and lower bounds. 


\subsubsection{Visual Inspection}

A visual inspection consists of comparing the mode shapes of the four experimental brace configurations to the mode shapes of the four brace configurations from a computer model. This situation is only applicable to experimental situations such as the Bridge House because the braces are removable. An example of a visual inspection is shown below.
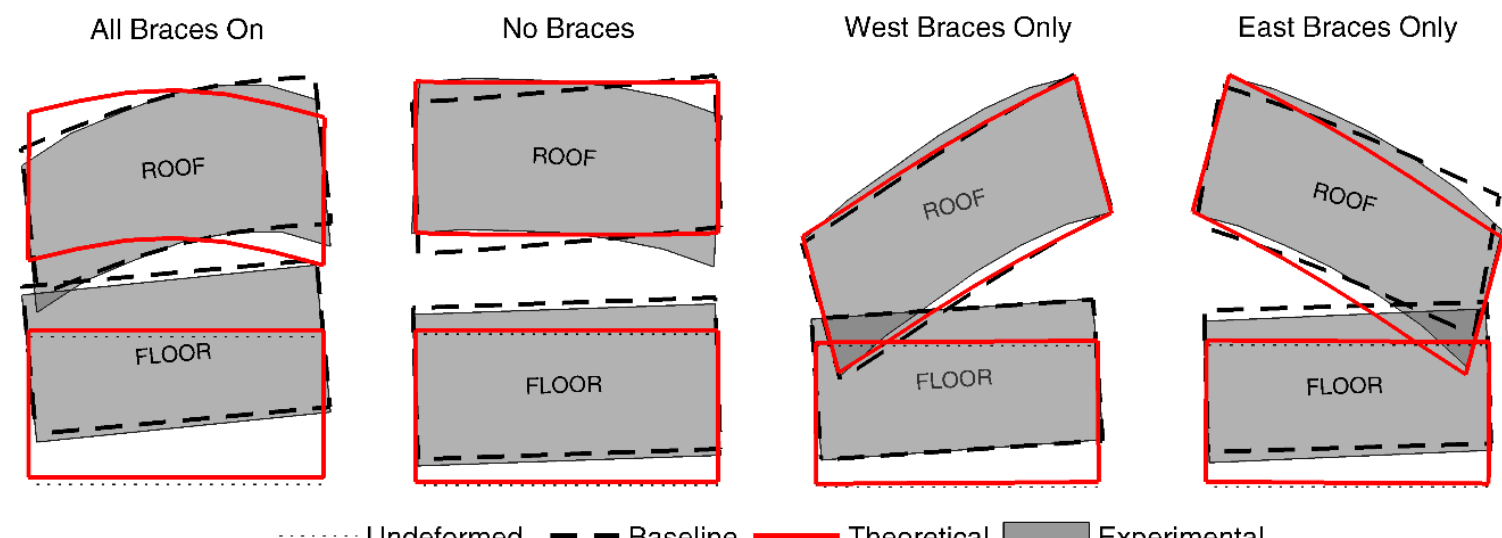

Figure 7-1: Example of visual inspection

The dashed line represents the mode shapes of the Baseline Model, the red line represents the results of Theoretical Model used for the parametric study, and the grey hatched area represents the mode shapes from the experimental data. Having the ability to compare all four experimental brace configurations against computer model results makes matching the brace configurations easy. This would not be possible in a post seismic event as only one experimental brace configuration would exist. A likelier example of visual inspection after an earthquake is the blind visual inspection.

\subsubsection{Blind Visual Inspection}

A blind visual inspection consists of examining the mode shape of one experimental brace configuration to the mode shapes of four brace configurations from a model. This method is similar to the process that would be done after a seismic event. This method will illustrate the 
difficulty in visually matching experimental results to theoretical results. An example is shown in Figure 7-2 below.

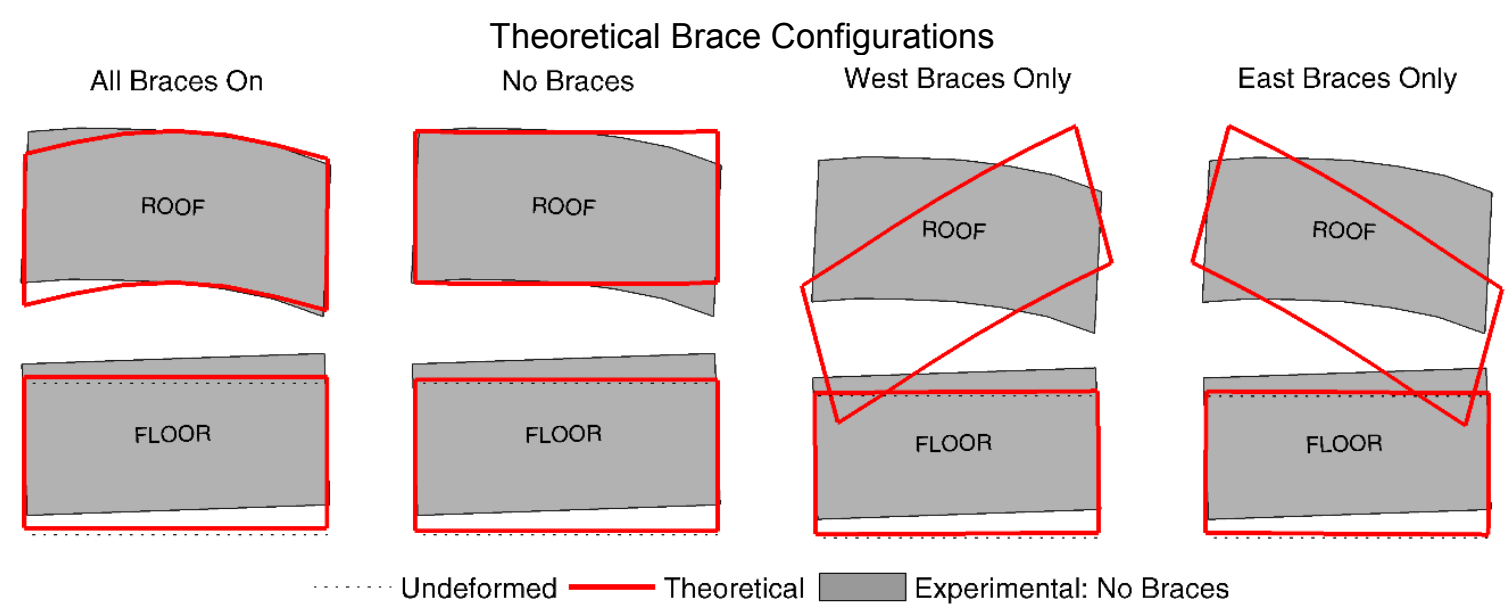

Figure 7-2: Example of blind visual inspection

The grey hatched area represents the mode shape of the No Braces experimental results. The red outline represents the mode shapes from four theoretical brace configurations outputted form a computer model. One would have difficulty in visually pairing the computer model results to the experimental results without additional damage detection parameters. The other method chosen is the Modal Assurance Criterion.

\subsubsection{Modal Assurance Criterion (MAC)}

To evaluate the correlation between mode shapes, the Modal Assurance Criterion (MAC) can be utilized. The Modal Assurance Criterion is a qualitative method that compares a suite of vectors against another suite of vectors of similar length. Because a mode shape is a vector represented in an array, the Modal Assurance Criterion can be used to compare various mode shapes. The MAC outputs a matrix is populated with numbers from 0 to 1 . Each cell in the matrix represents the comparison of two vectors. A cell with a value of 0 indicates that the compared vectors have no correlation, with a value of 0.5 indicates the compared vectors have a random correlation, and a cell with a value of 1 indicates a full correlation between compared vectors (Aleman2003). The equation of the Modal Assurance Criterion is shown below. 


$$
M A C_{i j}=\frac{\left(\varphi_{i}^{T} \varphi_{j}^{T}\right)^{2}}{\left(\varphi_{i}^{T} \varphi_{i}^{T}\right)\left(\varphi_{j}^{T} \varphi_{i}^{T}\right)}
$$

Where:

$i=$ the vector of the first mode shape compared

$\mathrm{j}=$ the vector of second mode shape compared

For the scope of this research, three variations of MACs will be established. They are listed below.

- Baseline Theoretical MAC: The $\phi_{i}$ term will always represent experimental mode shapes due to the respective brace configuration and the $\phi_{j}$ will always represent the brace configuration mode shapes from the baseline model. There are four baseline brace configurations compared against four experimental brace configurations; this will yield a total of 16 MAC values.

- Experimental MAC: Will compare experimental brace configurations against one another; both $\phi_{i}$ and $\phi_{j}$ will represent the results from experimental testing. The Experimental MAC creates ideal results and indicates how closely each brace configuration correlates with one another. This creates a symmetrical matrix with unity on the diagonals.

- Theoretical MAC: Will compare a theoretical model from a parametric study against the experimental results. The theoretical model will be a derivation from the baseline model and will study how changes impacts the results. For the Theoretical MAC, the $\phi_{i}$ term will represent experimental mode shapes due to the respective brace configuration, and the $\phi_{j}$ will always represent the mode 
shapes from the theoretical model. A summary of all three MAC definitions are below.

Table 7-2: MAC definitions

\begin{tabular}{|c|c|}
\hline MAC & Definition \\
\hline $\begin{array}{c}\text { Baseline Theoretical } \\
\text { MAC }\end{array}$ & Experimental Results vs Baseline Model \\
\hline Experimental MAC & Experimental Results vs Experimental Results \\
\hline Theoretical MAC & Experimental Results vs Theoretical Model \\
\hline
\end{tabular}

\subsubsection{Mass Weighted Modal Assurance Criterion (MWMAC)}

Several variations of the Modal Assurance Criterion are used for particular applications. One in particular, the Mass Weighted Modal Assurance Criterion (MWMAC), is helpful for two reasons. First, the MWMAC places priority on degrees-of-freedom with larger mass. Second, it allows for the multiple degrees-of-freedom with different units within the mode shape vector. The MWMAC equalizes the mass for the respective units in the mode shape vector before any matrix cross products are conducted. Typically, this allows for rotational and translational degrees-of-freedom within the same MWMAC calculation (Archer, 2014). The MWMAC is shown below in Equation 7-2.

$$
M W M A C_{i j}=\frac{\left(\varphi_{i}^{T} M \varphi_{j}^{T}\right)^{2}}{\left(\varphi_{i}^{T} M \varphi_{i}^{T}\right)\left(\varphi_{j}^{T} M \varphi_{j}^{T}\right)}
$$

For the Bridge House, rotational degrees-of-freedom were generally avoided in favor of translation degrees of freedom. The first mode behavior of the Bridge House is overwhelmingly transverse in the North-South direction and limiting analysis to translational degrees-of-freedom simplified analysis. However, the impact on rotational degrees of freedom in lieu of translation will be studied latter. Unless noted otherwise, all MAC analyses will be done using the Mass Weighted Modal Assurance Criterion. 


\subsubsection{Application of MAC}

In this research, four experimental brace configurations are being compared against four theoretical brace configurations. The MAC for these tests will be a four-by-four matrix with sixteen total values. It is the goal of this research that within every row, the highest correlation will be on the diagonal. In other words, when examining each brace configuration (the rows in the MAC matrix), it is anticipated that MAC number closest to 1 is also on the diagonal. This indicates that the computer model brace configuration resembles its experimental brace configuration counterpart more than any other experimental brace configuration. An example of a MAC used in this research is shown in below.

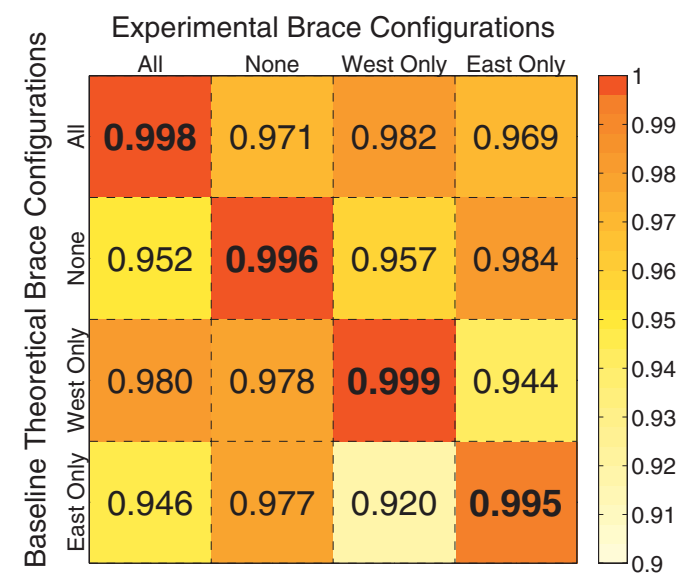

Figure 7-3: Example MAC

In real world application, there would only be one unknown experimental brace configuration reflecting the actual state of the structure but many theoretical brace configurations. This would yield a four-by-one MAC; it would have a total of four values. The highest value would indicate the highest correlation and the location of damage. Figure 7-3 has been revised below assuming there is only one experimental brace configuration, all other brace configurations have been blurred out; this information would be unknown to the experimenter. 


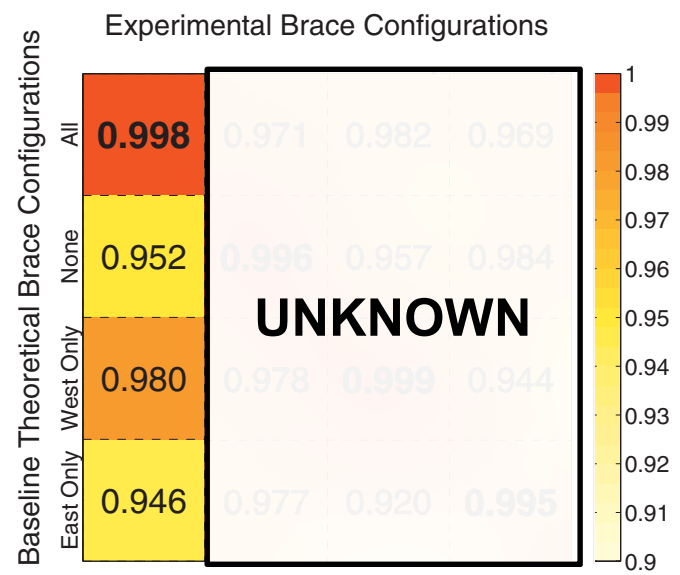

Figure 7-4: Real World Example MAC

The highest value is 0.998 and correlates to the All Braces On brace configuration.

Therefore, in real world application, one would conclude that the structure's brace configuration is All Braces On. 


\subsection{DAMAGE DETECTION}

\subsection{Baseline Model}

\subsubsection{Overview}

The Baseline Model will be compared to the first mode Experimental Results to determine if damage detection is possible. The techniques used will be a frequency comparison, a visual inspection, and a MAC analysis.

\subsubsection{Frequency Analysis}

The frequency of each brace configuration from the Baseline Model closely resembles the respective brace configurations from the Experimental Results. While this cannot be used to identify damage, it does yield important information. Figure 8-1 shows the experimental frequency sweep and the theoretical results. The dotted line represent frequencies predicted by the Baseline Model.

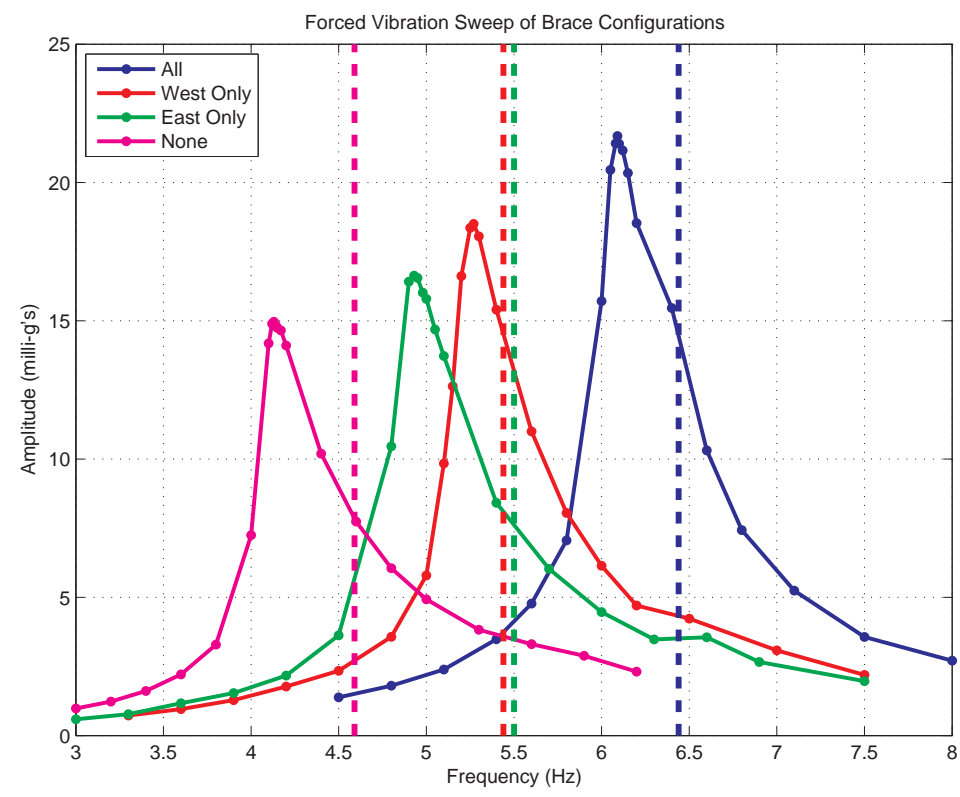

Figure 8-1: Frequency sweep with theoretical results 
Table 8-1 summarizes these results below.

Table 8-1: Baseline frequency comparison

\begin{tabular}{|c|c|c|c|}
\hline Brace Configuration & $\begin{array}{c}\text { Experimental } \\
\text { Frequency }(\mathrm{Hz})\end{array}$ & $\begin{array}{c}\text { Theoretical } \\
\text { Baseline } \\
\text { Frequency }(\mathrm{Hz})\end{array}$ & $\begin{array}{c}\text { Ratio } \\
\text { Theoretical/Experimental }\end{array}$ \\
\hline All Braces On & 6.09 & 6.44 & 1.06 \\
\hline West Only & 5.27 & 5.44 & 1.03 \\
\hline East Only & 4.93 & 5.50 & 1.16 \\
\hline No Braces & 4.13 & 4.59 & 1.11 \\
\hline
\end{tabular}

The percent differences are small which verifies the model accuracy. Additionally, it gives bounds as to which brace configuration is present. For example, after an earthquake, if the Experimental Results yielded a result of $6.09 \mathrm{~Hz}$ (the All Braces On configuration), by looking at the frequencies of the Baseline Model, a reasonable assumption could be established that any brace configuration could apply except for the No Braces configuration. The reason for this conclusion is that the model's frequency $(4.59 \mathrm{~Hz})$ is far off from the experimental $(6.09 \mathrm{~Hz})$. All the other brace configurations yielded frequencies that were reasonably close $(6.44 \mathrm{~Hz}, 5.44$ $\mathrm{Hz}$, and $5.50 \mathrm{~Hz}$ ). A frequency analysis helps establish reasonable bounds and helps with model verification although there is no qualitative measure to establish correlation.

\subsubsection{Visual Inspection}

The mode shapes of the Baseline Model compared against experimental results are shown below. These mode shapes are infinity normalized to one for each brace configuration. 


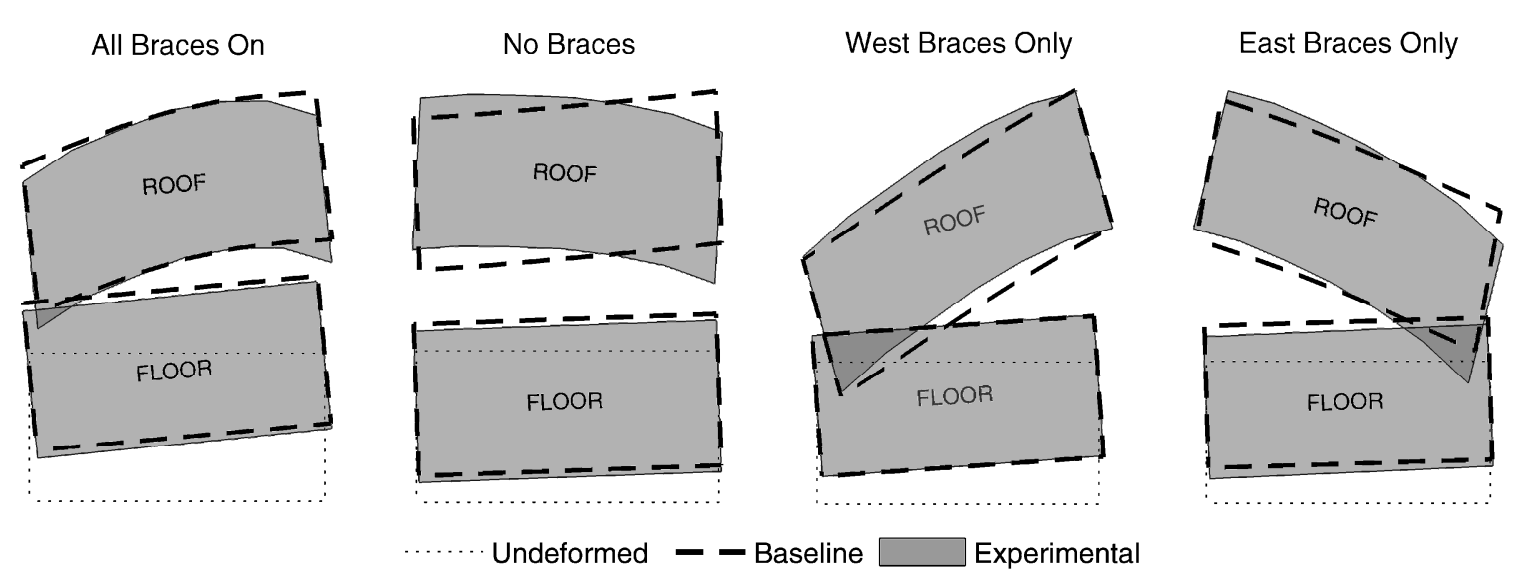

Figure 8-2: Baseline and Experimental Mode Shapes

By examining the normalized experimental mode shapes to the normalized theoretical mode shapes, it is clear that the Baseline Model captures the real behavior of the Bridge House. One discrepancy is observed. The No Braces configuration shows differences in the roof behavior between Baseline Model and the experimental results. It is expected that the exposed piers on the East side of the Bridge House allow for more flexibility and larger displacements than on the West side of the Bridge House. This is expected and observed in the roof behavior for the All Brace configuration and in the floor behavior for every brace configuration. However, this is not seen in the roof behavior in the No Brace configuration. This is attributed to the stiffness of the additional non-structural elements on the East side of the Bridge House that are not accounted for in the Baseline Model.

The floor behavior of the Baseline Model and the experimental results match closely with regards to story drift, rotation, and shape. This indicates the substructure calibration was accurate. The deflection in the roof diaphragm for all brace configurations is similar between the experimental and theoretical results; although, it appears as if the Theoretical Models have a slightly less roof deflection relative to the floor. This is likely caused from errors in estimating the stiffness of the interior built-up columns or from an error in the roof diaphragm calibration. 
Overall all, the estimate used from the calibration works well and should be considered a successful method to estimate the behavior of a diaphragm.

It was also discovered that the diaphragm behaves like a flexible diaphragm. In the All Brace configuration, reasonably large deflections are observed in the diaphragm compared to the story drift. When the No Brace configuration is tested, the diaphragm still deflects, but far less. Diaphragm deflection is dependent on the lateral force resisting system.

\subsubsection{Blind Visual Inspection}

A blind visual inspection illustrates the additional difficulty in visually predicting brace configurations. The Experimental Results for the All Braces On configuration are plotted against the results from the Baseline Model below.

Theoretical Brace Configurations

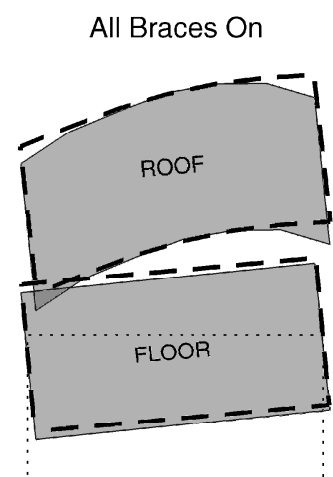

No Braces

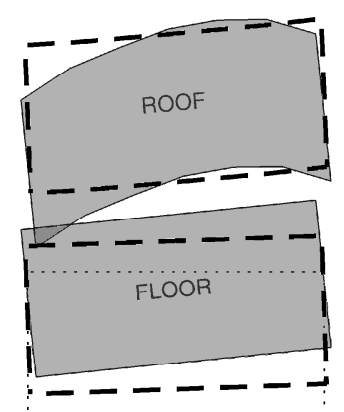

Undeformed - Baseline
West Braces Only

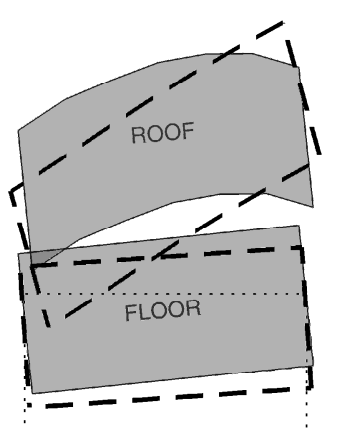

Experimental: All Braces On
East Braces Only

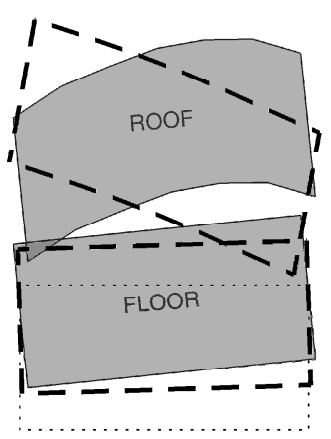

Figure 8-3: Blind visual inspection example 1

Visually in Figure 8-4: Blind visual inspection example 2, the All Braces On configuration Baseline Model most closely matches the All Braces On experimental results. The second most similar brace configuration is the No Braces. For this reason, a blind visual inspection done with the No Braces Experimental Results is shown below in Figure 8-4. 


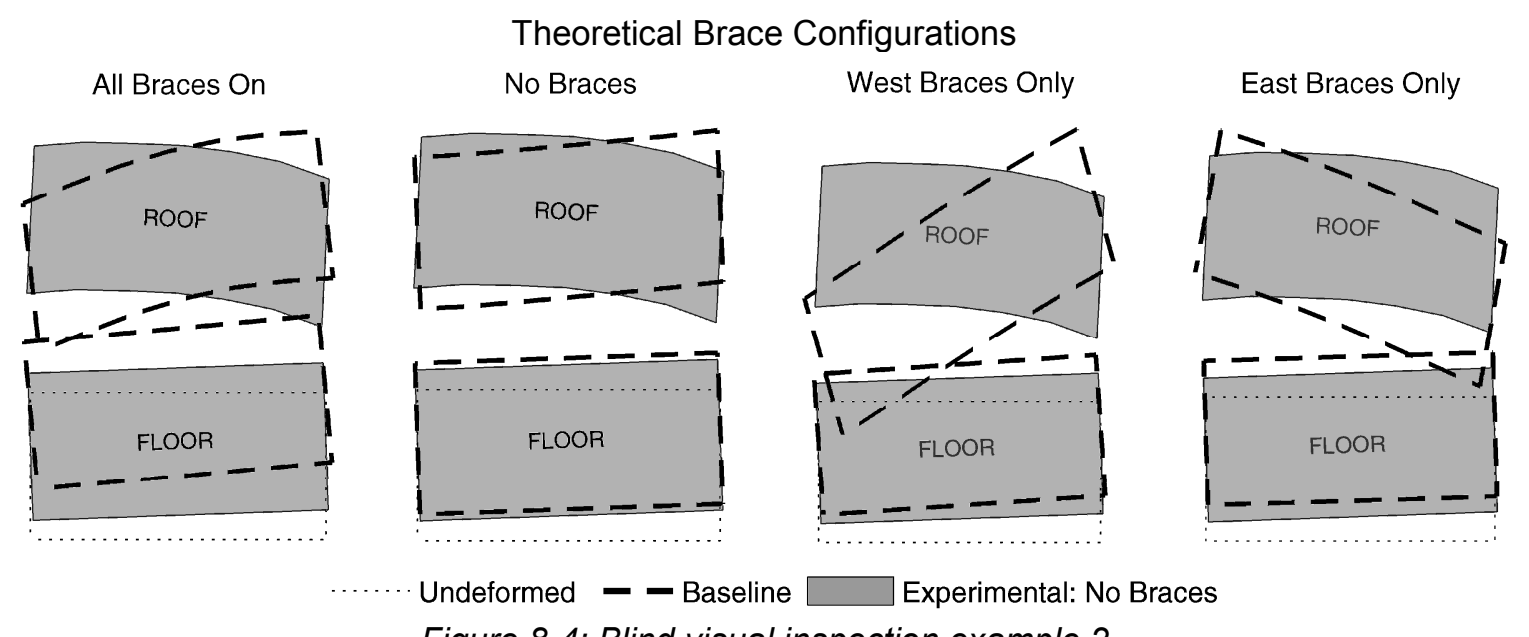

Figure 8-4: Blind visual inspection example 2

The blind visual inspection done for the No Braces configuration is slightly harder to predict, but the most reasonable assumption is that the No Braces Baseline Model configuration is the closest resemblance to the Experimental Results. Another metric which would help establish similarity between model and experimental brace configurations should be used. To assist in this verification, the Modal Assurance Criterion will be utilized.

\subsubsection{Modal Assurance Criterion}

A visual inspection shows that the first mode shapes of the brace configurations from the experimental results look similar to one another. Using the Modal Assurance Criterion, a MAC can be established to show the numerical correlation between the different brace configurations known as the Experimental MAC. The off-diagonal values will establish how similar the brace configurations are to one another. The further away the values are from one, the less correlation they have with the compared mode shapes. 


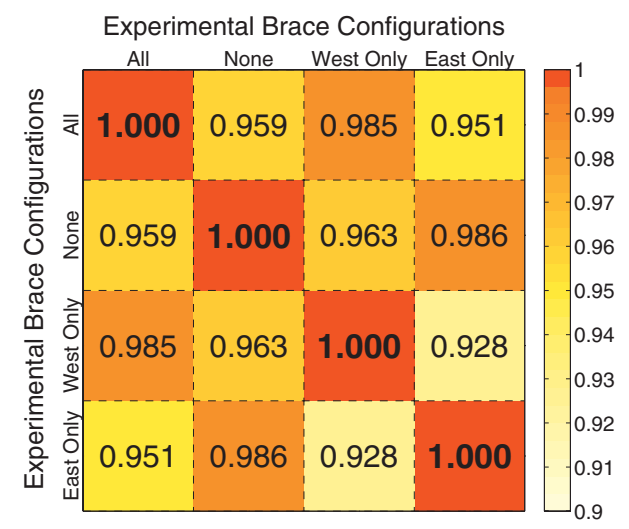

Figure 8-5: Experimental MAC

For each brace configuration, the next highest values are in the range of 0.985 to 0.986 .

This indicates that every brace configuration has a high similarity to at least one other brace configuration. The lowest value found on the off-diagonal are between Wests Only and East Only brace configurations. These MAC values are 0.928 . This value indicates that the least similar brace configurations have a high level of similarity.

Because of the similarity between the mode shapes of each brace configuration, the only way to establish and compare MACs is to compare the individual values against a Baseline $M A C$ against an Experimental MAC as shown in Figure 8-6.
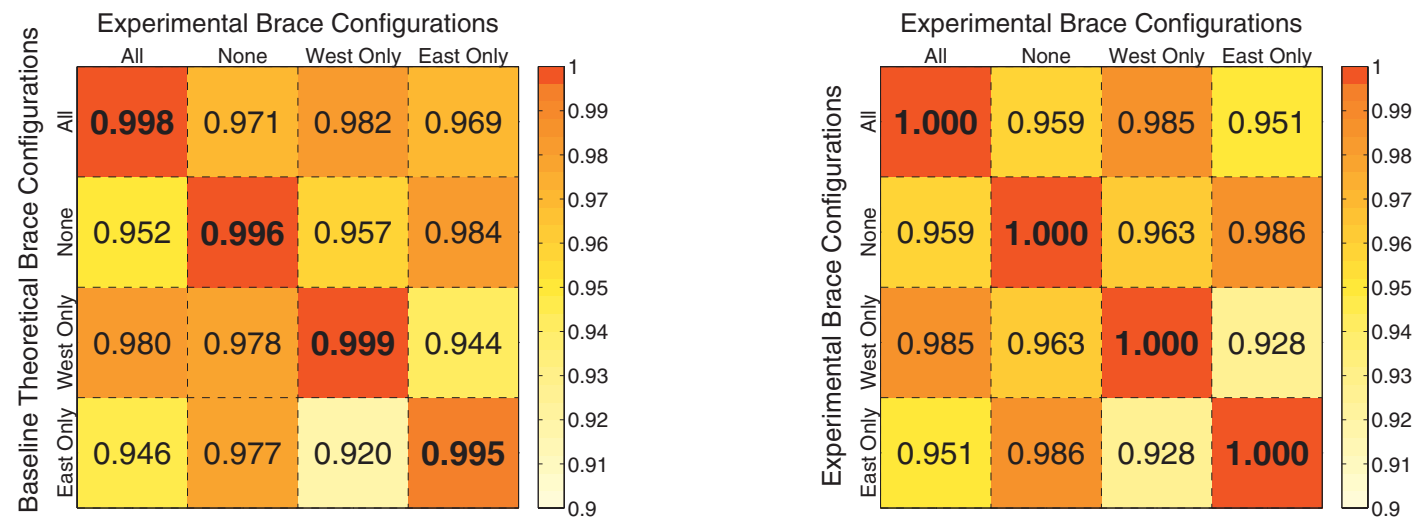

Figure 8-6: Baseline MAC (left), Experimental MAC (right) 
Figure 8-6 shows that the Baseline MAC has high correlations on the diagonal. The MAC correctly identifies Baseline Model brace configurations to the Experimental Results brace configurations. As expected, the off-diagonal values are all above 0.9. Note the color-bar scale is set between 0.9 and 1.0 , this scale was necessary to quickly identify difference between values. By visual inspection, the off-diagonal values of the Baseline MAC uncannily resemble the Experimental MAC. This is further indication that the Baseline Mode/ resembles the Experimental Results; although it is possible the brace configuration mode shapes are so similar that any theoretical model that has mostly translational behavior will yield similar results. This will be studied further in parametric studies. In section 8.2 , all parametric studies will be compared against the Baseline Theoretical MAC.

\subsubsection{Conclusion}

Using the foundation and roof parameters found from experimental testing, damage detection was successful. Frequency analysis, visual inspections, and a MAC analysis give a suite of tools to help identify Experimental Results to the corresponding Baseline Model. The frequency analysis gives bounds as to expected frequencies. In this study, it showed greatest differences in the All Braces On and the All Braces Off configurations. The MAC values correctly identified all four brace configurations although strong correlations resulted throughout the No Braces Experimental Results. This is where a visual inspection and the frequency analysis can help clarify any uncertainties. Now that damage detection has been shown to be successful, several parametric studies to the model will be conducted to address uncertainty during modeling.

System identification was also successful. The floor behavior matches well for every brace configuration. The displacements of the roof match closely however it appears the diaphragm displacement values are slightly off and yielded slightly stiffer roof diaphragm values 
than the Experimental Results. The system identification and damage detection were considered successful.

\subsection{Model Parametric Studies}

\subsubsection{Overview}

The following section is a series of parametric studies that change one or more of the model properties. The order of the studies are outlined below.

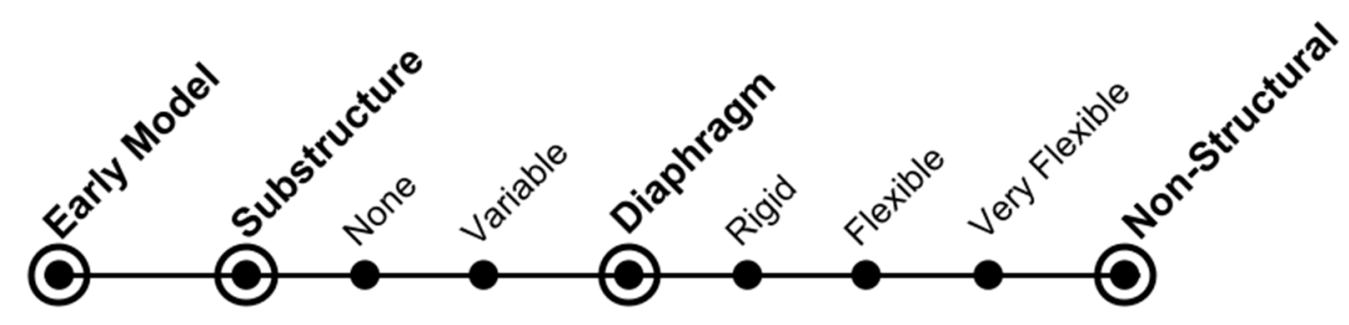

Figure 8-7: Parametric studies

\subsubsection{Early Model}

\subsubsection{Overview}

Analysis was done on an early computer model before the Baseline Model was established. Both models had similar geometry however values for the roof diaphragm, built-up sections, and substructure properties were picked by judgement and iterative methods. The model also had modeling errors. For example, test loads were applied that should have yielded symmetrical reactions and distributions of forces. The Early Baseline Model did not distribute these test loads symmetrically. This model was made by the author as an undergraduate student for another class. After the shortcomings of these results were discovered, the Baseline Model. Using the Early Theoretical Model helps establish and show the consequences of using an inferior model. 


\subsubsection{Visual Inspection}

The results of the early Theoretical Model compared to the Baseline Model and Experimental Results are shown in Figure 8-8.
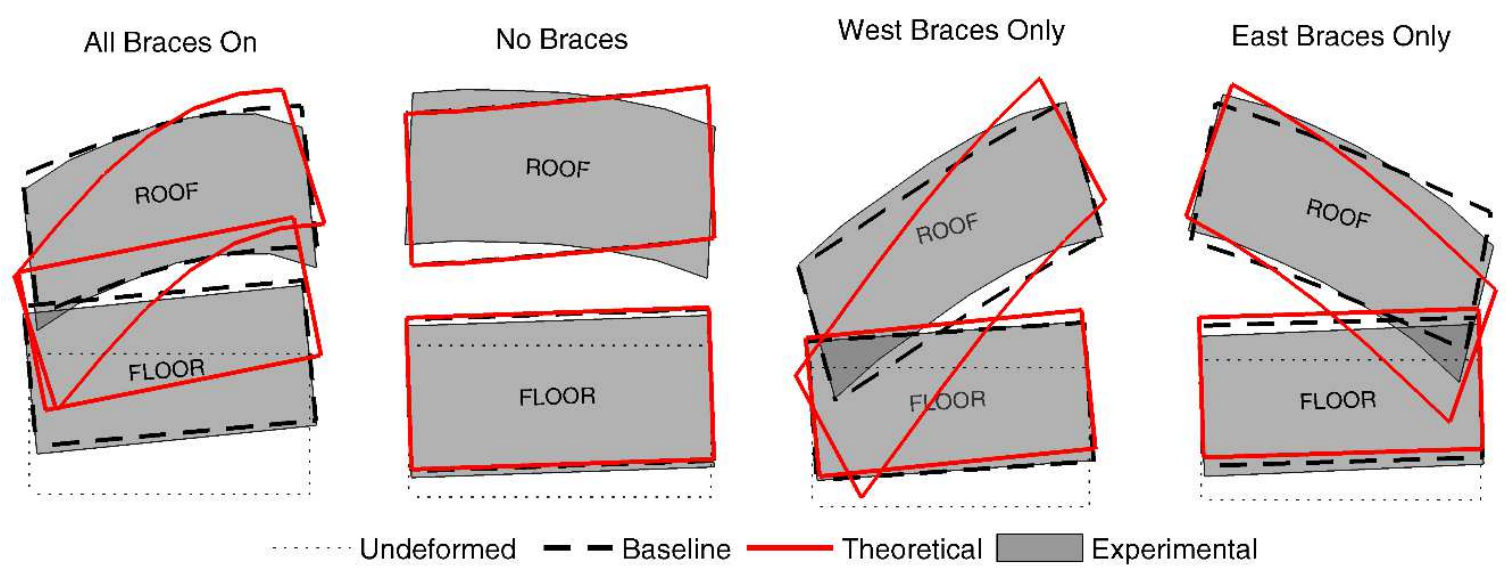

Figure 8-8: Early model mode shapes

Figure 8-8 shows that the Early Theoretical Model was able to resemble both the Baseline Model and Experimental Results. In the No Brace configuration, both models are almost identical; all other brace configurations had larger differences in both roof displacements, floor displacements, and inter-story drift. The Early Theoretical Model had many exaggerations in mode shapes compared to the Baseline Model. These differences exaggerate the differences in mode shapes and makes it visually plausible to correlate brace configurations. To determine if these exaggerations are significant, a MAC analysis is necessary.

\subsubsection{MAC Comparison}

When looking at the experimental mode shapes, the Early Theoretical MAC had the largest values on the diagonal; the Early Theoretical Model was successful in detecting correlations as shown in Figure 8-9: Early Theoretical MAC (left), Baseline MAC (right). 

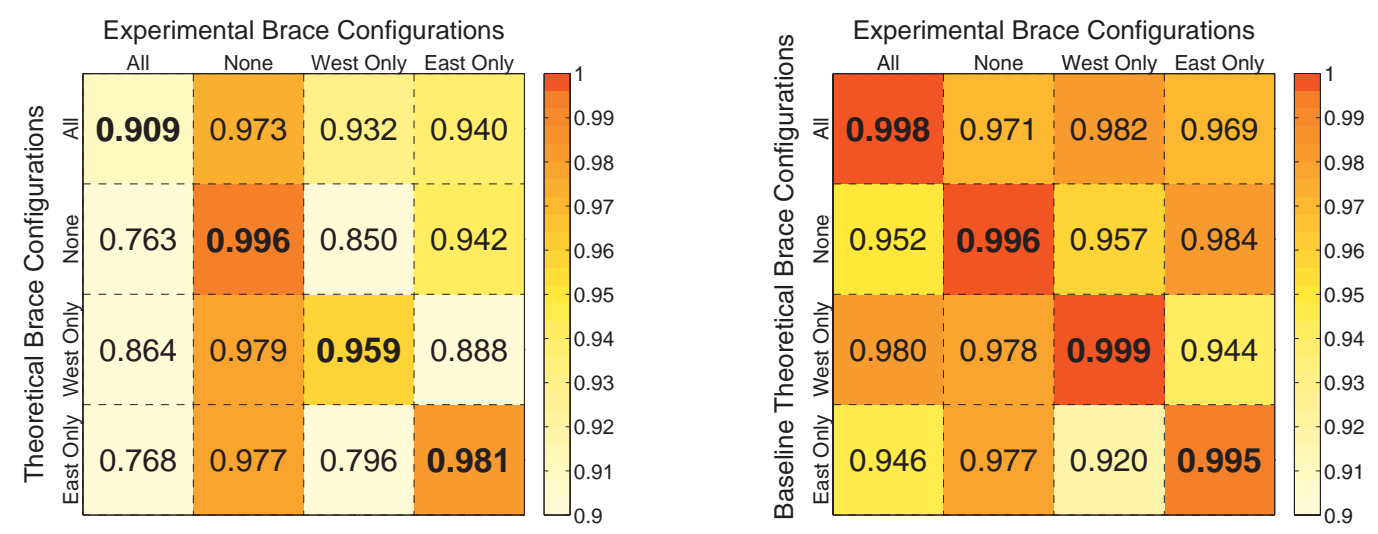

Figure 8-9: Early Theoretical MAC (left), Baseline MAC (right)

While the largest values are on the diagonal for the Early Theoretical MAC, the results were further from unity and, in some cases, substantially less than the diagonals in Baseline MAC. This suggests that the early Theoretical Model was less successful in finding correlations than the Baseline MAC. This is expected as there was limited grounds for some modeling decisions. The off-diagonal values are much less than the off-diagonal values in the Baseline MAC. In some cases, the values were below 0.9 and 0.8 . This suggests that the Early Theoretical Model was "better" at predicting non-correlation.

The Early Theoretical MAC producing smaller off-diagonal values than the Baseline $M A C$ or Experimental MAC is unexpected. If there is a case of lower off-diagonal values in the Theoretical MAC than the Experimental MAC, then the model is less precise, despite doing a "better" job of minimizing correlation between different conflicting brace configurations. Typically in modal analysis, this is thought of as a preferable situation. In reality, it is impossible to have a realistic model that is "better" than experimental situations. MACs have a wide range of uses, and in modal analysis they are typically used to compare mode shapes from the same model against each other. The method of comparing a suite of different models is unique to this research. The assumption that low off-diagonal values yield preferable results is a 
misconception. High diagonal values should be the only valid metric when using MACs for this purpose.

\subsubsection{Early Model Conclusion}

These results indicate that a rudimentary model is capable of producing correlations that correctly identify the experimental brace configurations. Even though the off-diagonal values varied, the diagonal values still showed correlations to a relatively high level. These results are promising to support that MACs can be used to compare analytical and experimental results to detect damage. Additionally, off-diagonal values in the MAC should not be used as a correlation metric (or lack of correlation metric).

\subsubsection{Substructure - Rigid}

\subsubsection{Overview}

A model was established that had no substructure springs. No substructure behavior was modeled and the boundary conditions were fixed. The rational for choosing this model was based upon an early assumption that the substructure properties were not necessary.

\subsubsection{Visual Inspection}

The results of the model with fixed substructure springs are shown in Figure 8-10.
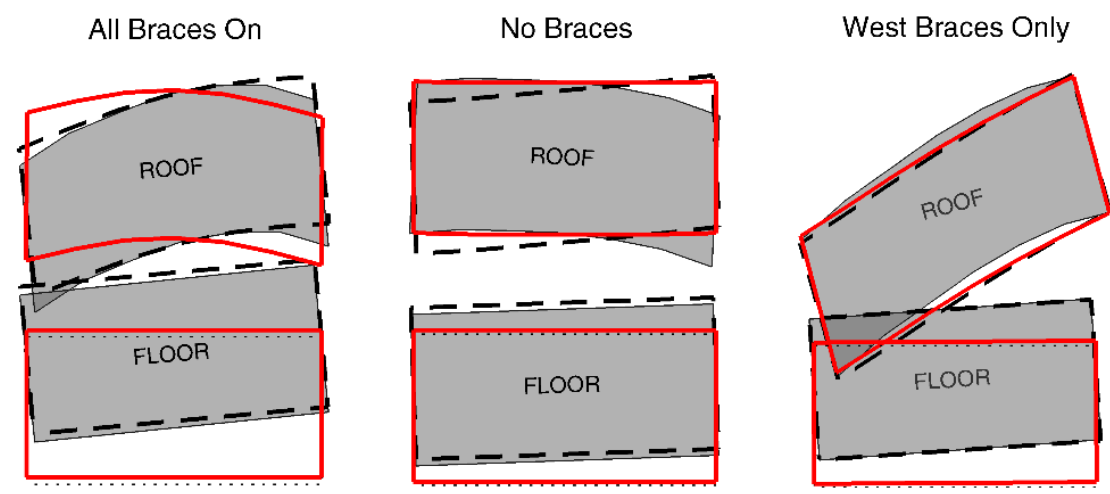

East Braces Only

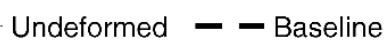

Theoretical

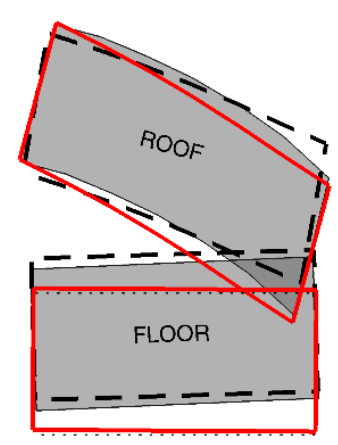

Figure 8-10: Rigid substructure mode shapes 
The results of the Rigid Substructure Theoretical Model capture the roof behavior relatively well in three of the four brace configurations. It does not capture the roof behavior well in the All Braces On configuration. This is due to the symmetry of the model when there are no sub-soil springs. As expected, the floor behaviors for this study do not match the experimental values at all. The floor deflections are all almost identical in all brace configuration due to the fact the floor has fixed restraints. Additionally, the floor moves slightly even though the substructure is fixed. This occurs because the theoretical values taken from the model were at the top of the floor slab, whereas the fixed boundary conditions were at the bottom of the columns. There is approximately a six to eight inch difference in elevation between the boundary conditions and the top of the floor slab.

\subsubsection{Blind Visual Inspection}

In a real world environment, experimental data from only one brace configuration would be available, making the comparison much harder. Both Figure 8-11 and Figure 8-12 represent the difficulty in visually matching mode shapes in a poor model by plotting one experimental mode shape against four theoretical mode shapes.

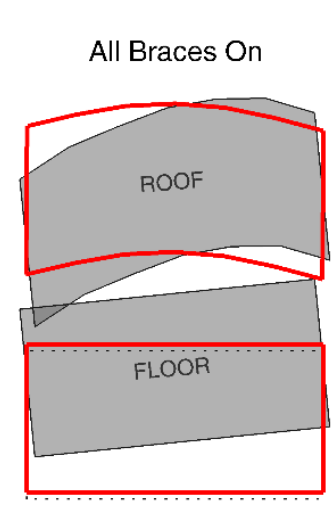

Theoretical Brace Configurations No Braces $\quad$ West Braces Only East Braces Only
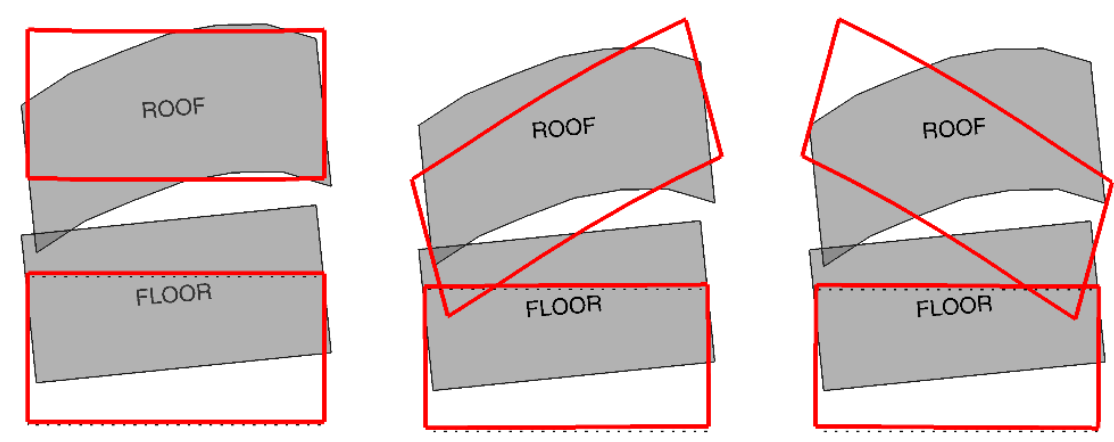

Undeformed Theoretical Experimental: All Braces On

Figure 8-11: Blind visual inspection example 3 
All Braces On
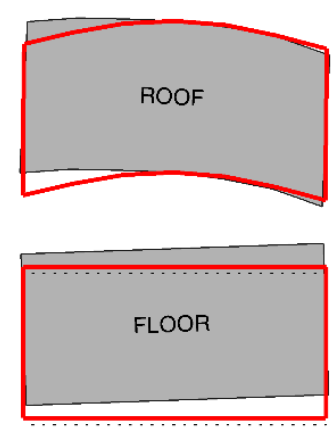

Theoretical Brace Configurations

No Braces
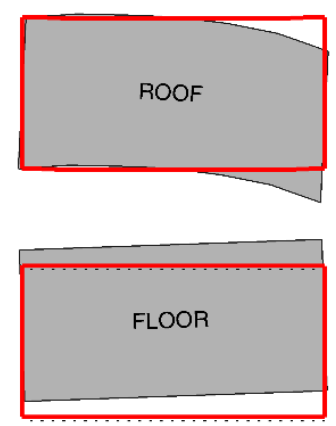

Undeformed
West Braces Only

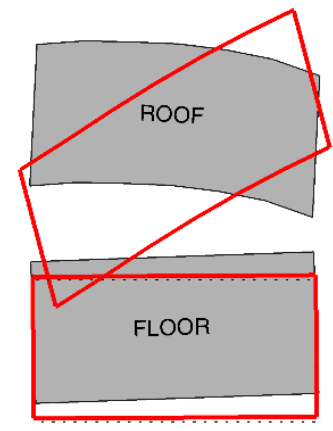

Experimental: No Braces
East Braces Only

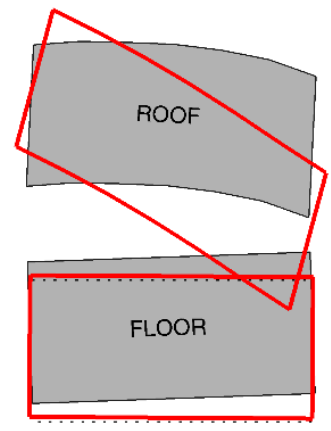

Figure 8-12: Blind visual inspection example 4

In Figure 8-11, the matching mode shapes are the No Brace configurations. Clearly, the All Braces On configuration would also be a reasonable guess. In Figure 8-12, the matching mode shapes are the All Braces On brace configuration. Every brace configuration but the West Braces Only configuration could arguably be an acceptable guess. By taking out all experimental brace configurations except for one, it makes much harder to visually correlate theoretical behavior to the experimental behavior.

\subsubsection{MAC Comparison}

The Theoretical MAC only identifies two of the four brace configurations as shown in Figure 8-13. All MAC values were lower than the Baseline MAC. Interestingly, the off-diagonal values were also lower. This is further evidence that using the off-diagonal values is not a good metric for predicting brace configurations. 

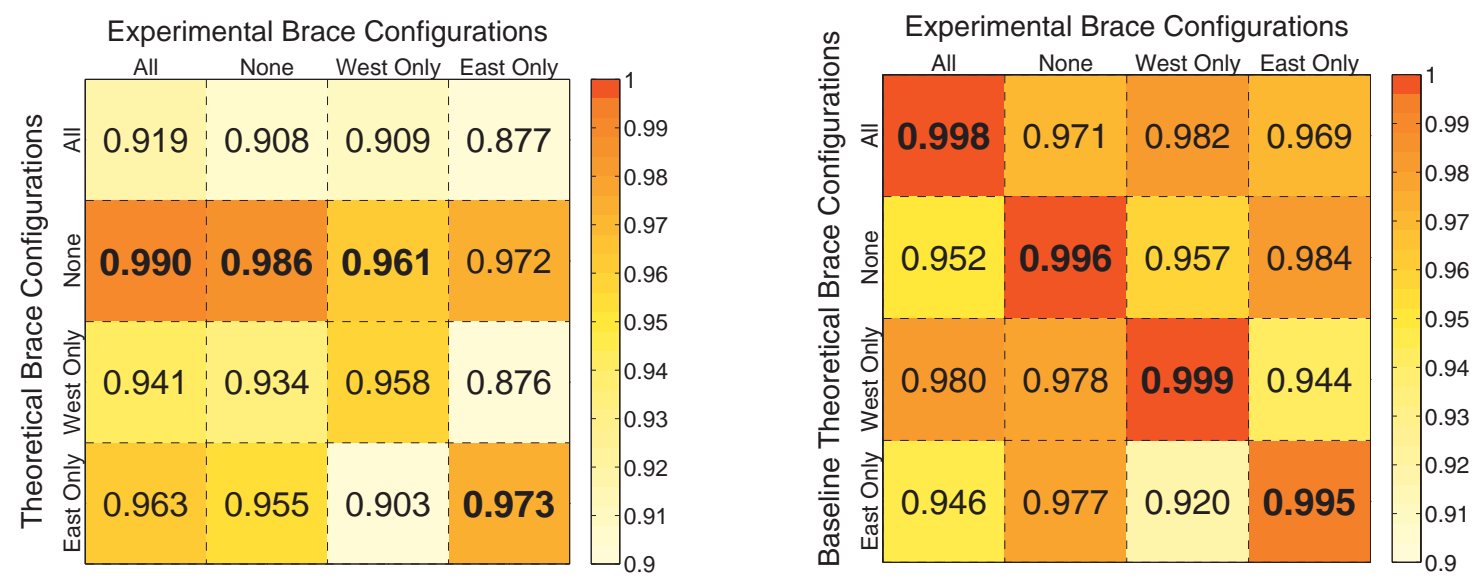

Figure 8-13: Rigid Substructure Theoretical MAC (left), Baseline MAC (right)

\subsubsection{Rigid Substructure Conclusion}

For the Bridge House, correct modeling of the substructure behavior is crucial for identifying the differences between the brace configurations. Because the mode shapes are predominantly translational, the rotational contribution due to the substructure springs helps distinguish the various brace configurations. The behavior between stories yields information that is valuable in defining mode shapes and should always be accounted for.

\subsubsection{Substructure Springs - Variable and Individual}

\subsubsection{Overview}

During system identification, it was discovered that the substructure behavior was dependent on the shaker amplitude and the mode shapes. The response of the substructure was non-linear based upon the amplitude of the shaker and brace configuration. Because of this, the substructure properties were averaged for the baseline model. In a post-seismic evaluation, one set of experimental data would exist, the state of the structure. To explore if the averaging used during research impacted the results, a model was made for each brace configuration. Each model had the respective substructure springs for each brace configuration. 


\subsubsection{Visual Inspection}

The impact that individual substructure springs had on their respective brace configurations compared to that of averaged substructure was negligible. Figure 8-14 shows that the difference is almost indistinguishable. A close examination between of the All Braces On configuration shows a small difference between the Baseline Model and the Theoretical Model. But essentially, this difference is negligible.

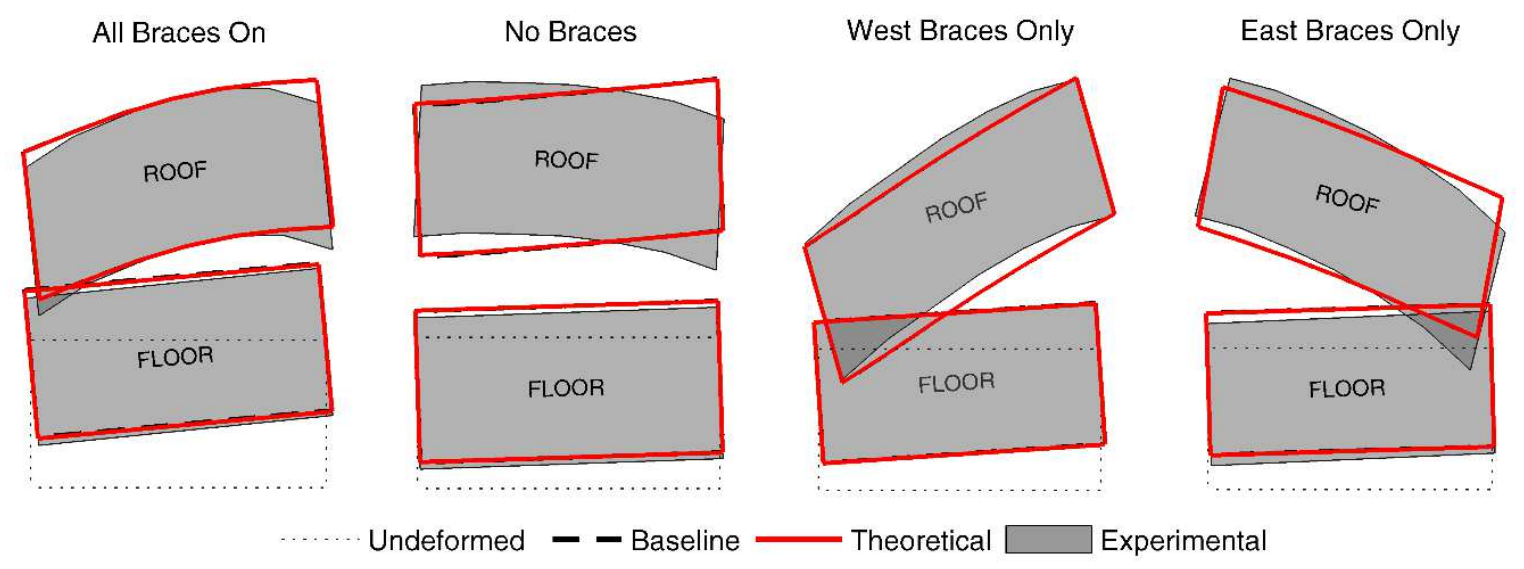

Figure 8-14: Variable substructure mode shapes

\subsubsection{MAC Comparison}

The Theoretical MAC was almost identical to the Baseline MAC. Changing the substructure springs made little difference as shown below in Figure 8-15.
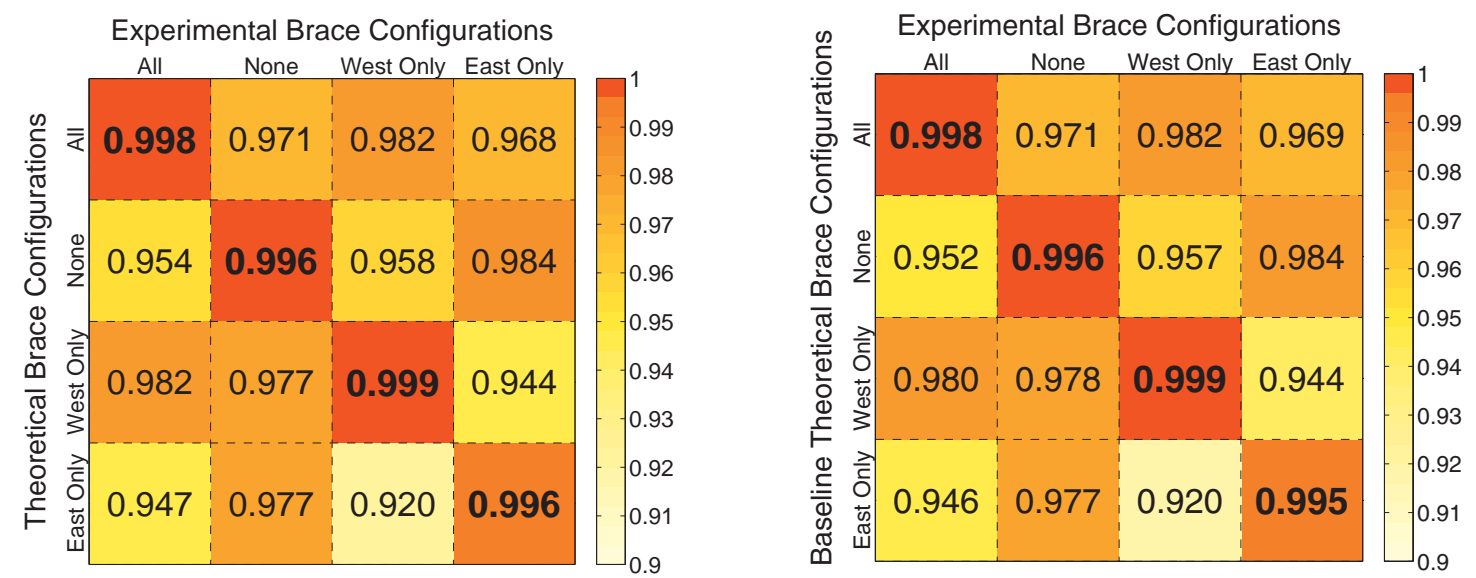

Figure 8-15: Variable Substructure MAC (left), Baseline MAC (right) 


\subsubsection{Variable Substructure Conclusion}

Exact substructure properties are not necessary for locating damage or for making a good model. A logical and verified estimate is necessary and acceptable. The Bridge House is unique as a substantial amount of its flexibility come from the substructure. Most structures are not as dependent on soil and foundation properties.

\subsubsection{Diaphragm Changes - Rigid}

\subsubsection{Overview}

An easy assumption during modeling is to assign the diaphragm as rigid as it eliminates many of the problems that are associated with modeling and predicting the roof behavior. To examine if a rigid roof approximation will capture the appropriate behavior, a parametric study was conducted.

\subsubsection{Visual Inspection}

Upon visual examination, it appears as if there is mild success as the Theoretical Model looks very similar to the Baseline Model. However, there are several similarities between the No Braces and East Braces Only configurations as well as the All Braces On and West Braces Only.
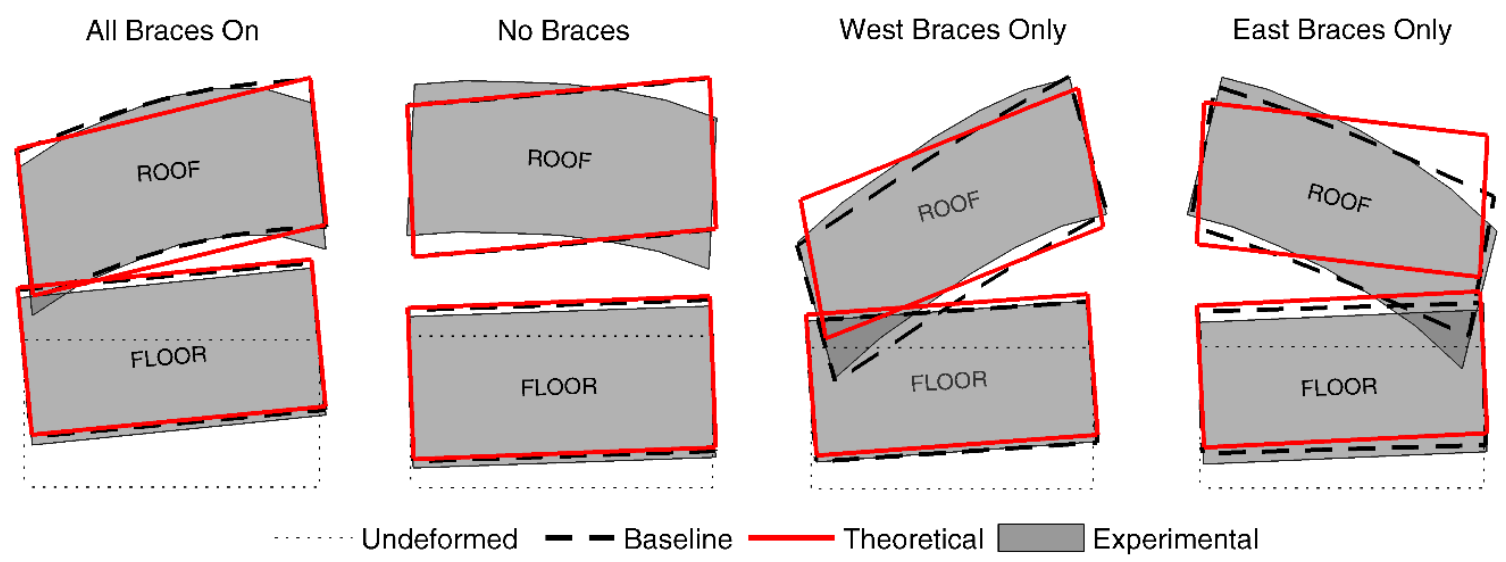

Figure 8-16: Rigid roof mode shapes 


\subsubsection{Blind Visual Comparison}

By removing the Baseline Model results and plotting each brace configuration against one experimental brace configuration, it becomes difficult to visually correlate brace configurations. Figure 8-17 shows the No Brace configuration against all four theoretical brace configurations for a rigid roof. Figure 8-18 shows the All Braces On configuration against all four theoretical brace configurations.

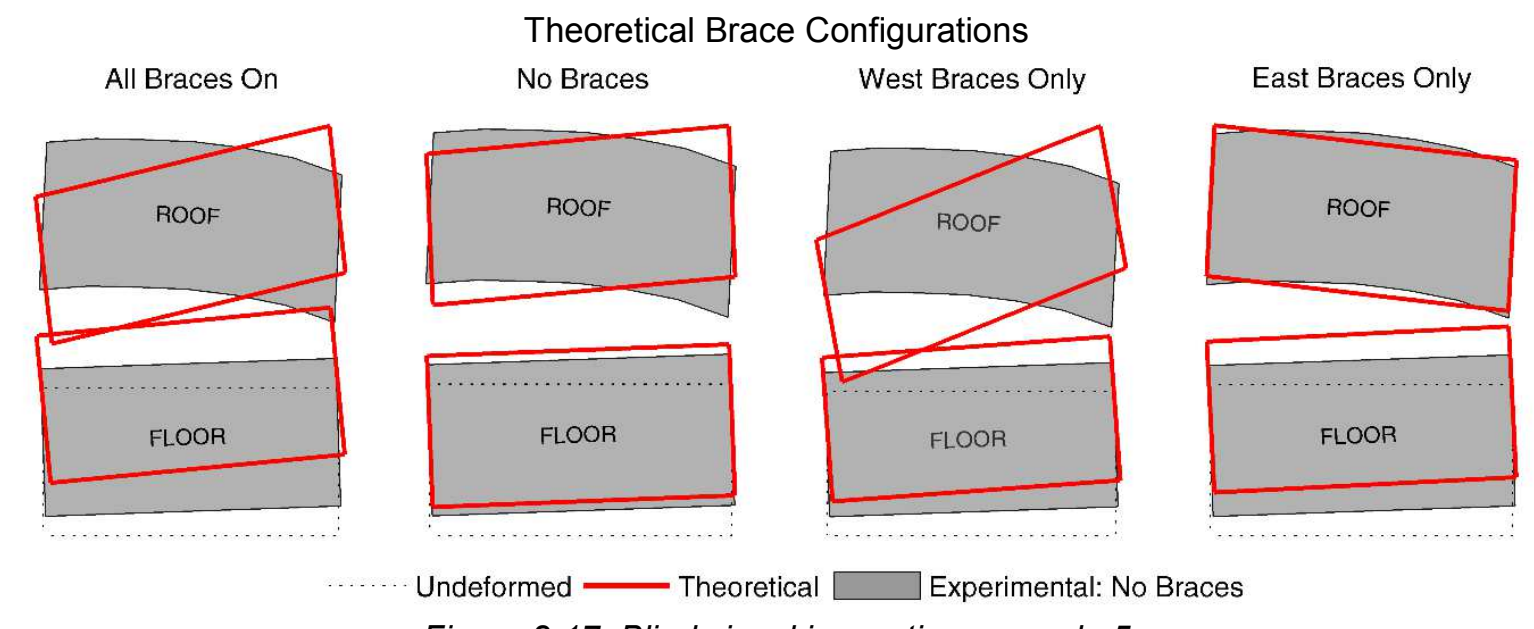

Figure 8-17: Blind visual inspection example 5

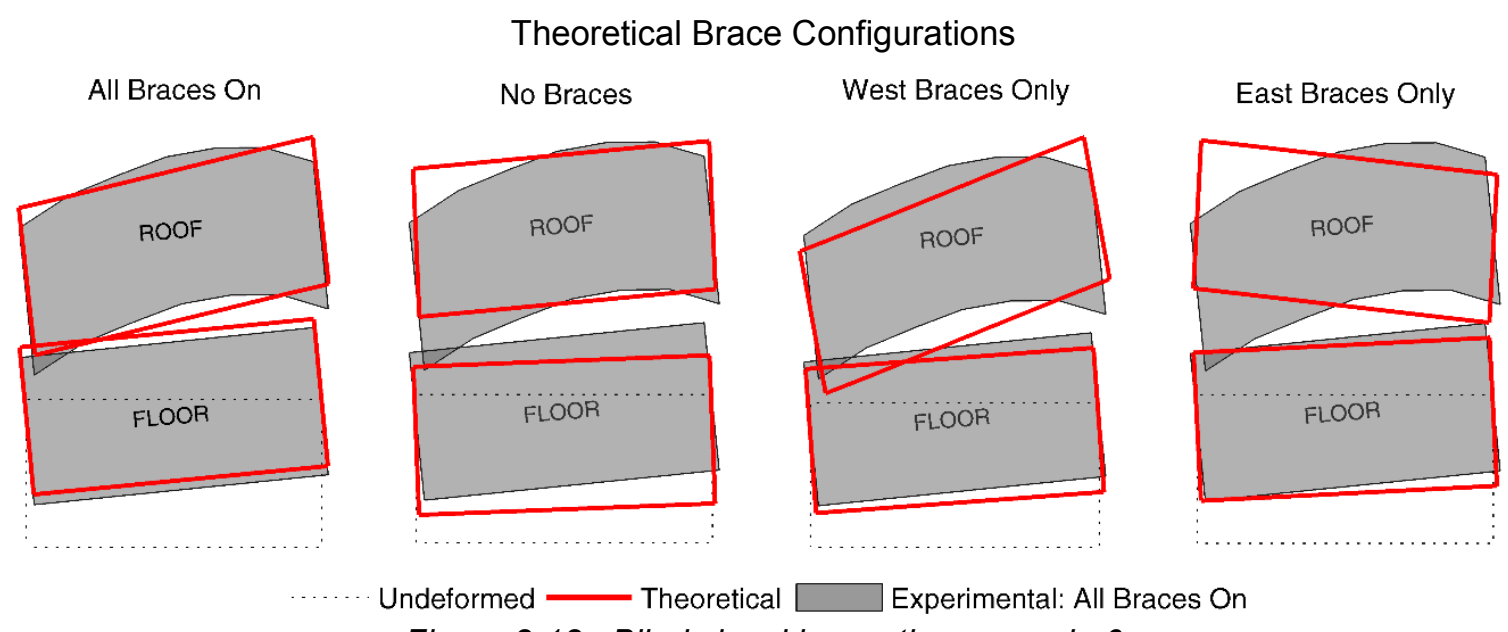

Figure 8-18: Blind visual inspection example 6 
These comparisons are similar to real world scenarios. It is difficult, if not impossible, to visually match the experimental values to the theoretical model with any degree of certainty. To qualitatively asses if there are any differences, a MAC analysis is necessary.

\subsubsection{MAC Comparison}
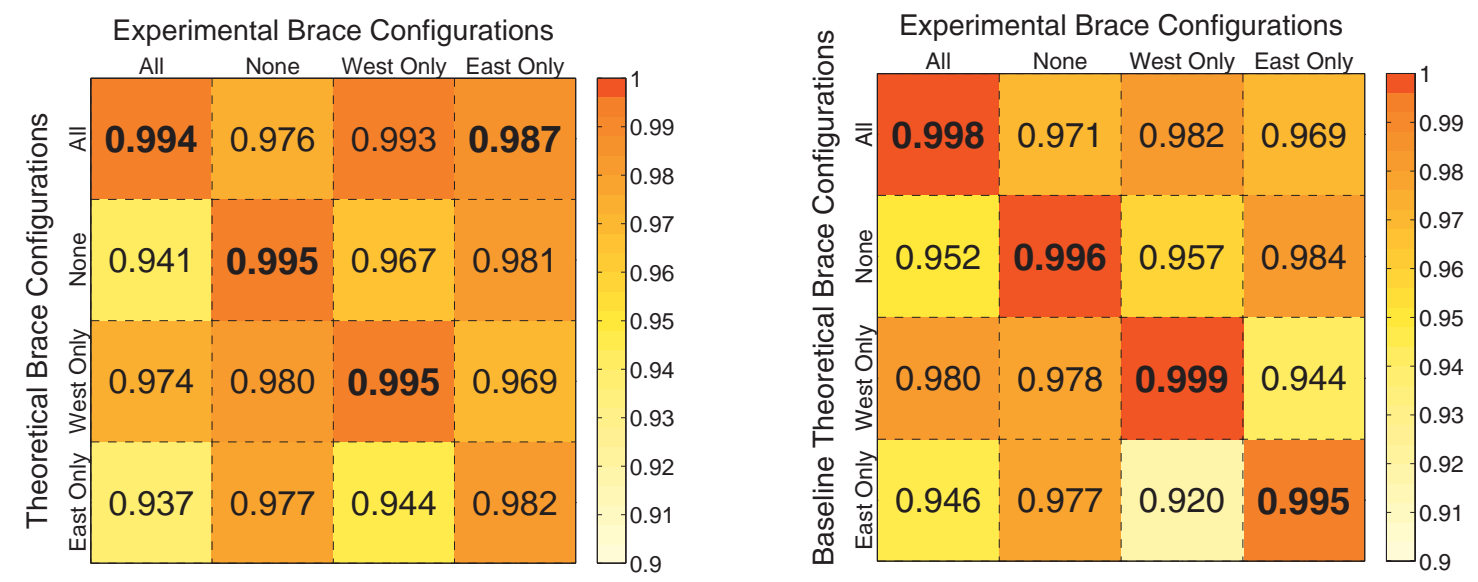

Figure 8-19: Rigid Roof Theoretical MAC (left), Baseline MAC (right)

The Theoretical MAC in Figure 8-19 correctly identified three of the four brace configurations. It was not capable of predicting the experimental East Only brace configuration. Generally, the Theoretical MAC was similar to the Baseline $M A C$, but its inability to predict all the brace configurations voids the rigid body assumption as an appropriate approximation of the roof behavior.

\subsubsection{Diaphragm Changes - Flexible}

\subsubsection{Overview}

A parametric study with a much more flexible diaphragm was established. An equivalent thickness of 0.008 inches was chosen for the shell elements instead of an equivalent thickness of 0.022 inches. All other properties were kept the same. This change represented an early attempt at calculating the diaphragm stiffness. 


\subsubsection{Visual Inspection}

Visually, it appears as if this Theoretical Model was as successful, if not more successful, than the Baseline Model. The All Brace On and the No Braces configuration match almost perfectly. The No Braces configuration did not change from the Baseline Model. This occurs because when the lateral system is sufficiently flexible, a roof will act rigid; decreasing the roof thickness by several factors still allows the roof to behave rigidly.
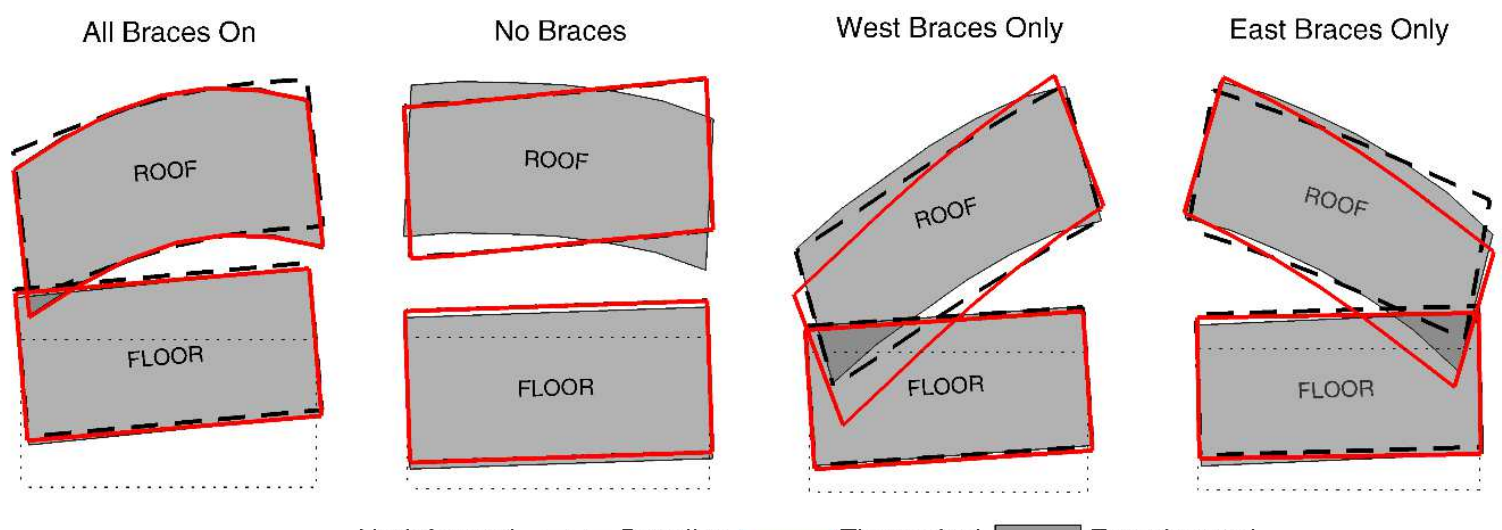

Undeformed - Baseline

Theoretical

Experimental

Figure 8-20: Flexible roof mode shapes

The West Braces Only and East Braces Only configurations changed yet closely resembled the Experimental Results. To examine the impact of this change qualitatively, a MAC analysis will be done.

\subsubsection{MAC Comparison}

The MAC analysis for a flexible roof Theoretical MAC is shown below. 

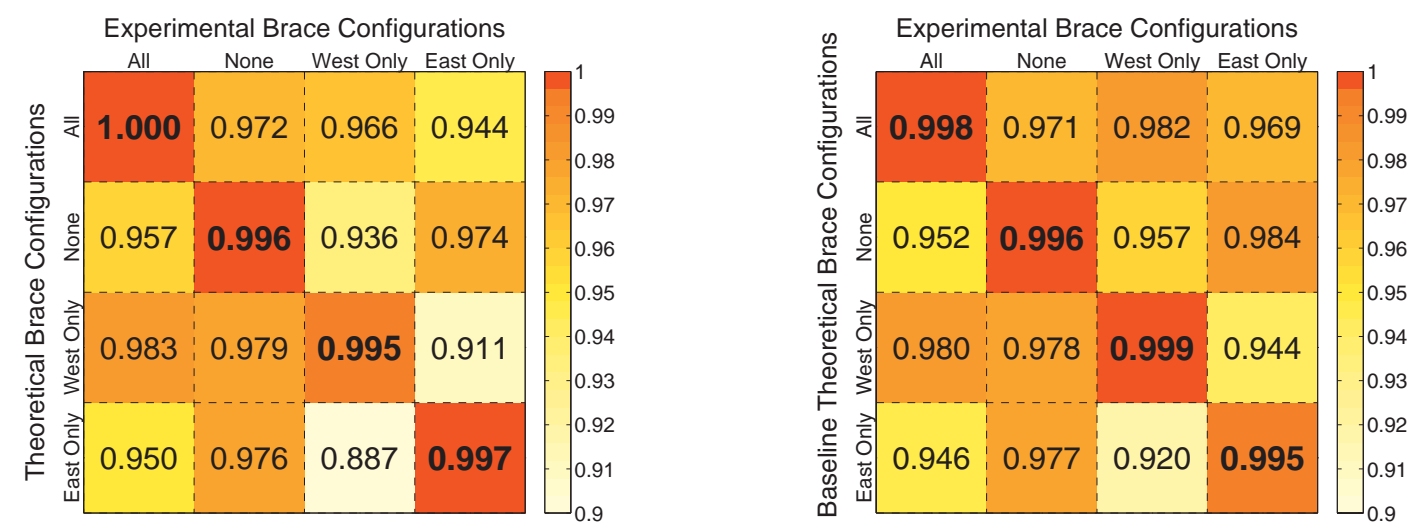

Figure 8-21: Flexible Roof Theoretical MAC (left), Baseline MAC (right)

The Theoretical MAC correctly predicted every brace configuration and yielded very similar results to the Baseline MAC both on the diagonal and on the off-diagonal. In the All Brace Configuration, the diagonal yielded a 1.000; this is the first full correlation found in the studies thus far. Because this modification appears to have created an equally sufficient model, the Theoretical MAC is compared to the Experimental MAC.
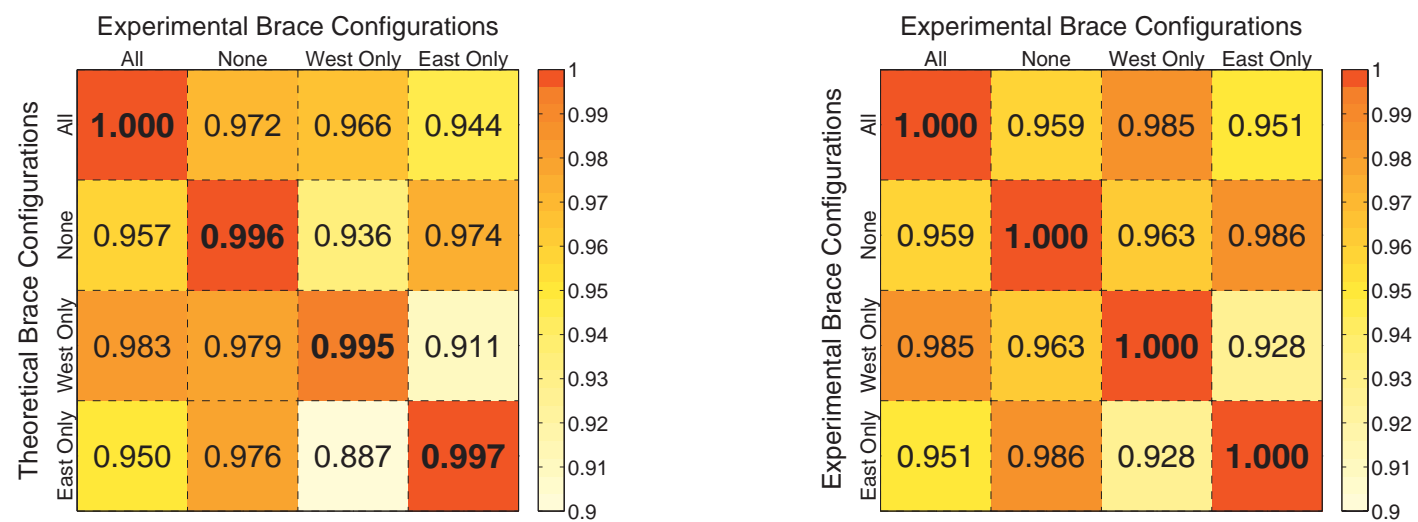

Figure 8-22: Flexible Roof Theoretical MAC (left), Experimental MAC (right)

Few differences are found between the Theoretical MAC and the Baseline MAC to the Experimental MAC. Individual MAC values can be recognized as different, but the overall behavior is the same. 


\subsubsection{Flexible Diaphragm Conclusion}

This study shows that even though the roof diagram can have a shell thickness of $1 / 2.75$ than that chosen for the Baseline Model, and the MAC is still able to find correlations between the brace configurations from Experimental Results and the respective brace configurations from the Theoretical Model. This significance indicates that that a model with vast differences in the roof properties is not only capable, but also adequate, for a reasonable analysis.

\subsubsection{Diaphragm Changes - Very Flexible}

\subsubsection{Overview}

Continuing the study of diagram flexibility on the results of damage identification, a new model with a diaphragm thickness of 0.004 inches, $1 / 5.5$ as thick than the Baseline Model, was made. The thickness of 0.008 inches yielded good results; it was proposed that a building with increased flexibility in the roof could do the same.

\subsubsection{Visual Inspection}

The very flexible Theoretical Model yielded similar results to the previous but with greater diaphragm deflections as shown in Figure 8-23 below.

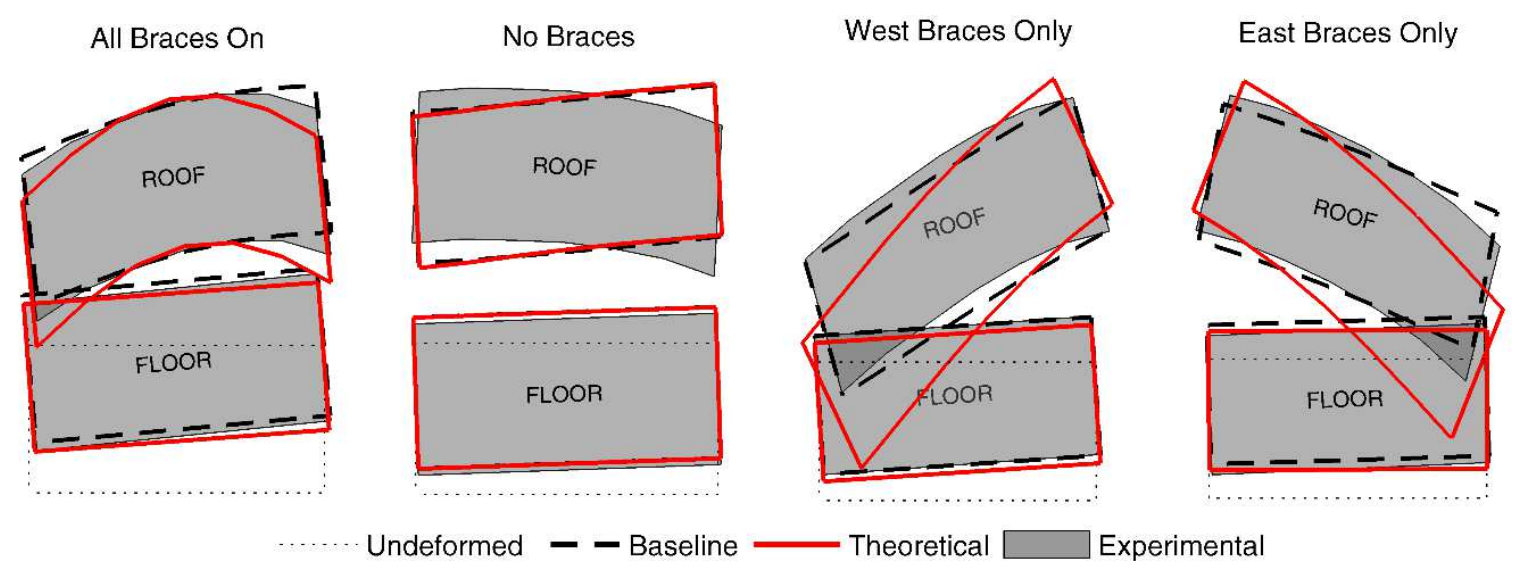

Figure 8-23: Very flexible roof mode shapes 
With this very flexible diaphragm, it is clear which braces correlate. In some cases, it appears that the diaphragm displacements are no longer small compared to the story drifts. Through all the roof studies, the No Brace configuration has been extremely stable to any changes in diagram stiffness. This is expected as the diaphragm acts rigid when lateral force resisting system is very flexible.

\subsubsection{Blind Visual Inspection}

In all other brace configurations, the Theoretical Model shows exaggerations in roof displacements. The impact on these exaggerations barely impacts a blind visual inspection. These exaggerations are shown Figure 8-24 and Figure 8-25; the All Braces On and the No Braces configurations, respectively, are plotted against the Theoretical Model Mode Shapes.
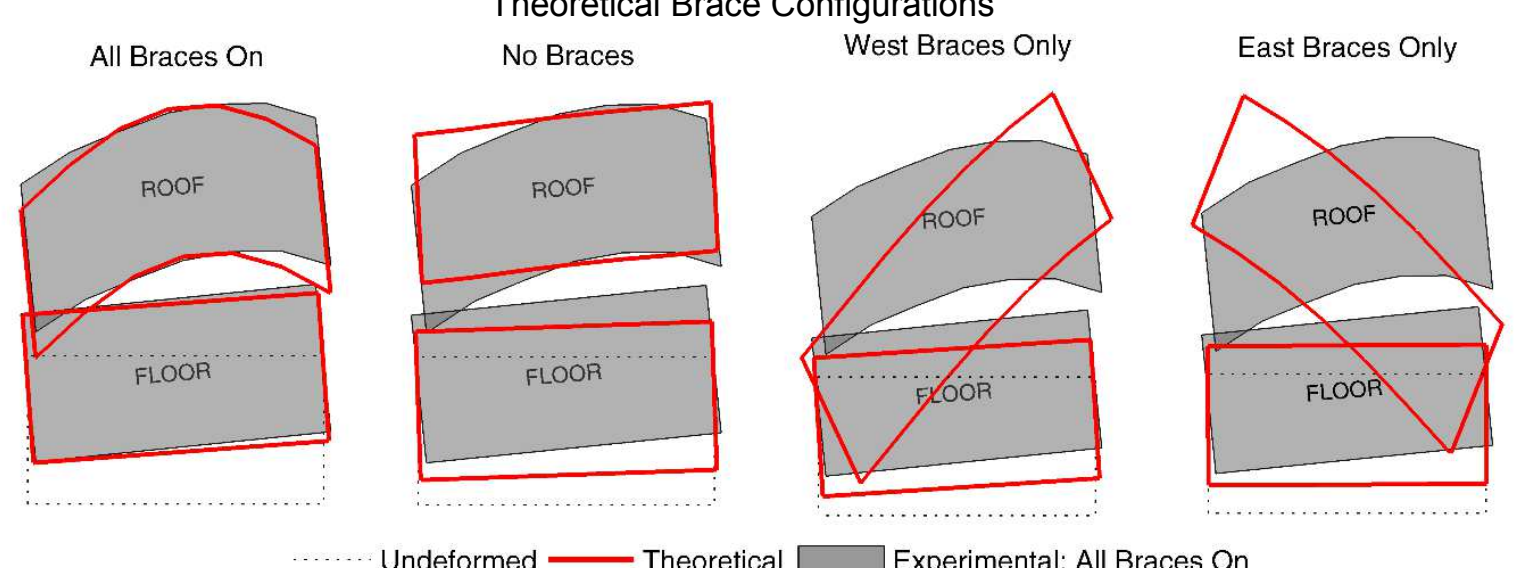

Undeformed Theoretical

Experimental: All Braces On

Figure 8-24: Blind visual inspection example 7 


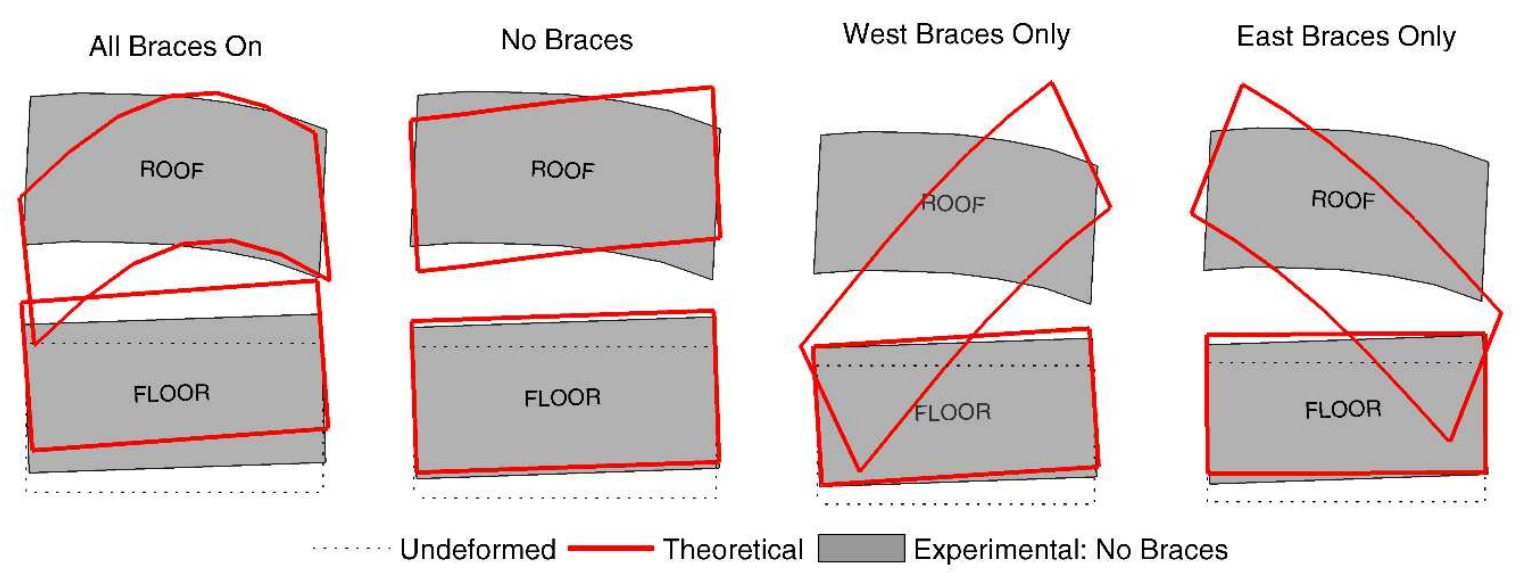

Figure 8-25: Blind visual inspection example 8

\subsubsection{MAC Comparison}

Even with the extreme exaggerations of the roof diaphragm, an examination of the MAC shows strong correlations. The Theoretical MAC correctly identifies all four brace configurations with only a slightly lower level of correlation than the Baseline Theoretical MAC. The offdiagonal values for the West Only and East Only brace configurations are significantly lower as well. As discussed previously, this is not a good indicator of lack of correlation. This occurs because the exaggerations in each brace line are amplified by the flexibility in the diaphragm and create more distinction between each brace configuration, hence lowering the values on the off-diagonal. Even with a diaphragm that is 5.5 less stiff then the Baseline Model, the MAC is still able to recognize the similarity between theoretical and experimental mode shapes.
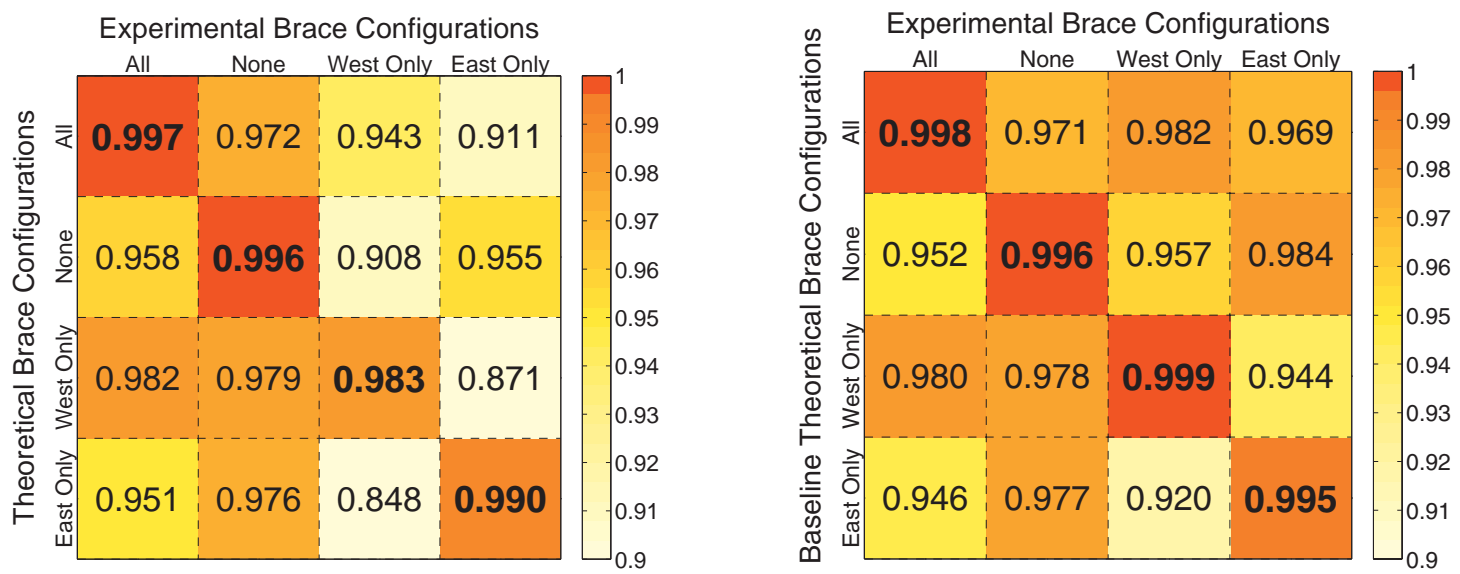

Figure 8-26: Very Flexible Roof Theoretical MAC (left), Baseline MAC (right) 


\subsubsection{Very Flexible Diaphragm Conclusion}

With a clearly wrong diaphragm stiffness value, the Theoretical MAC still produces strong values. Identification by visual inspection is more difficult but still feasible. This study shows how robust the MAC is in predicting brace configurations.

\subsubsection{Modeling of Non-Structural}

\subsubsection{Overview}

As previously discussed, the East Side of the Bridge House has substantial additional non-structural elements that contribute to the lateral stiffness of the structure. The stiffness of these elements is hard to quantify as the types of components range from steel spandrel mullions to plywood walls. When the braces are disengaged on the East Side, these nonstructural elements account for significant additional stiffness, as seen with the No Braces experimental results. To account for these elements, a model was made that estimated for these elements.

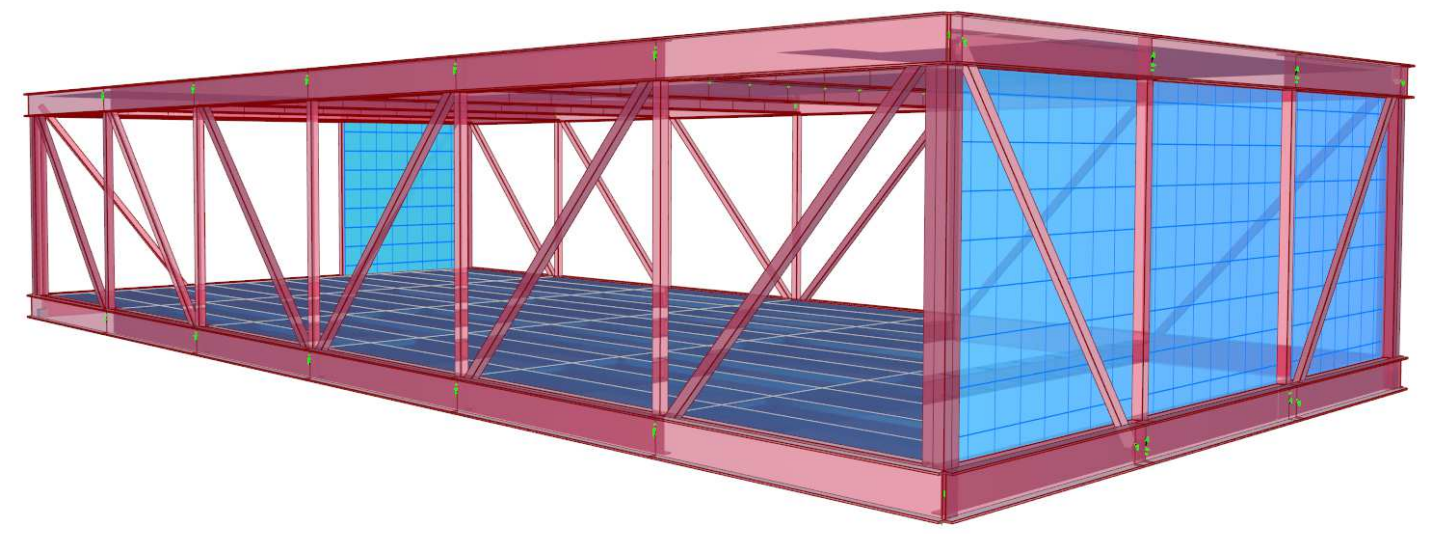

Figure 8-27: Non-structural model

Membrane elements that had the approximate lateral stiffness of a timber shear wall were added to both the East and West side of the Bridge House. Three times more elements were added to the East side of the Bridge House to represent the current condition. 


\subsubsection{Visual Inspection}

When plotting the Theoretical Results, it appears as if this change captured the irregularity experienced for the No Braces configuration as the theoretical roof displacements closely match the experimental. Additionally, all other brace configurations appear to closely match. Visually, this is perhaps the most accurate model yet, even more so than the baseline.

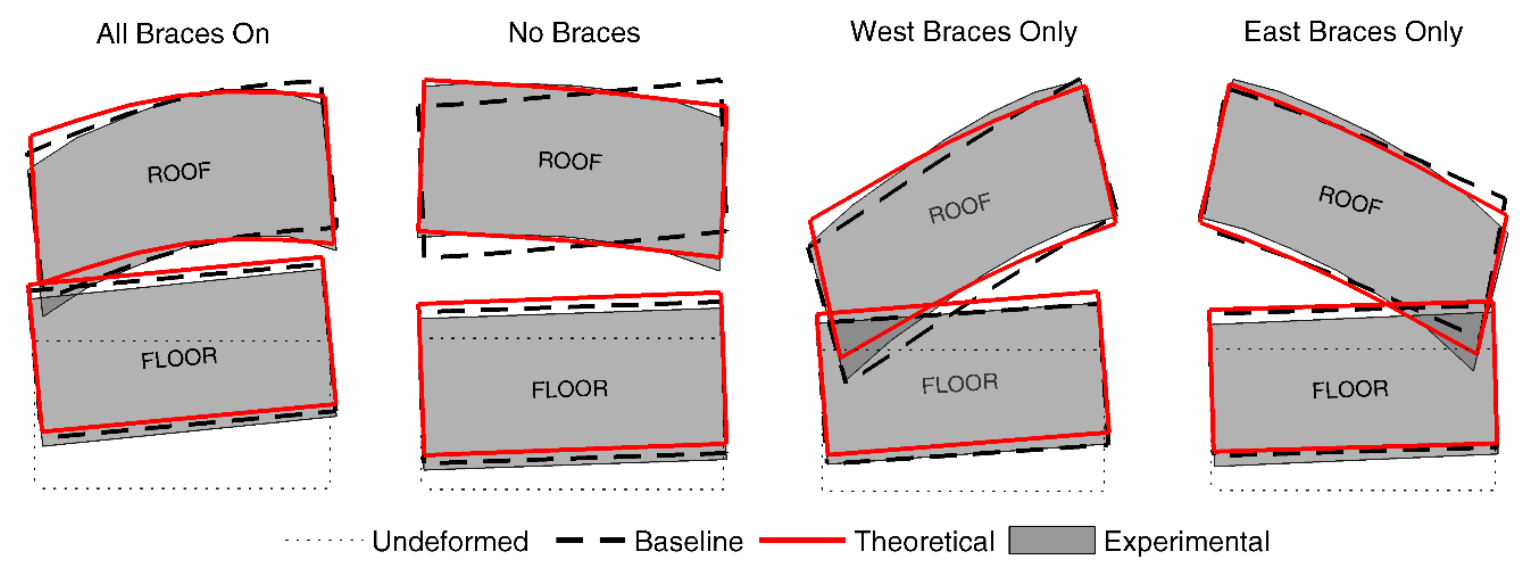

Figure 8-28: Increased non-structural mode shapes

Qualitatively, a MAC analysis must be done to verify that the changes to the model improved or matched the baseline results.

\subsubsection{MAC Comparison}

The Theoretical MAC is correctly able to identify the experimental results to the theoretical results for every brace configuration. The diagonal MAC values are very high, all larger than 0.995. When comparing the Theoretical MAC to the Baseline Theoretical MAC, it appears that the off-diagonal values of the Theoretical MAC, are noticeably higher. For example, in the West Only experimental brace configurations, the matched MAC value is 0.996, while the largest off- diagonal is 0.994 ; this shows a high level of correlation between the West Only experimental brace configuration and the All Braces On theoretical brace configuration. 

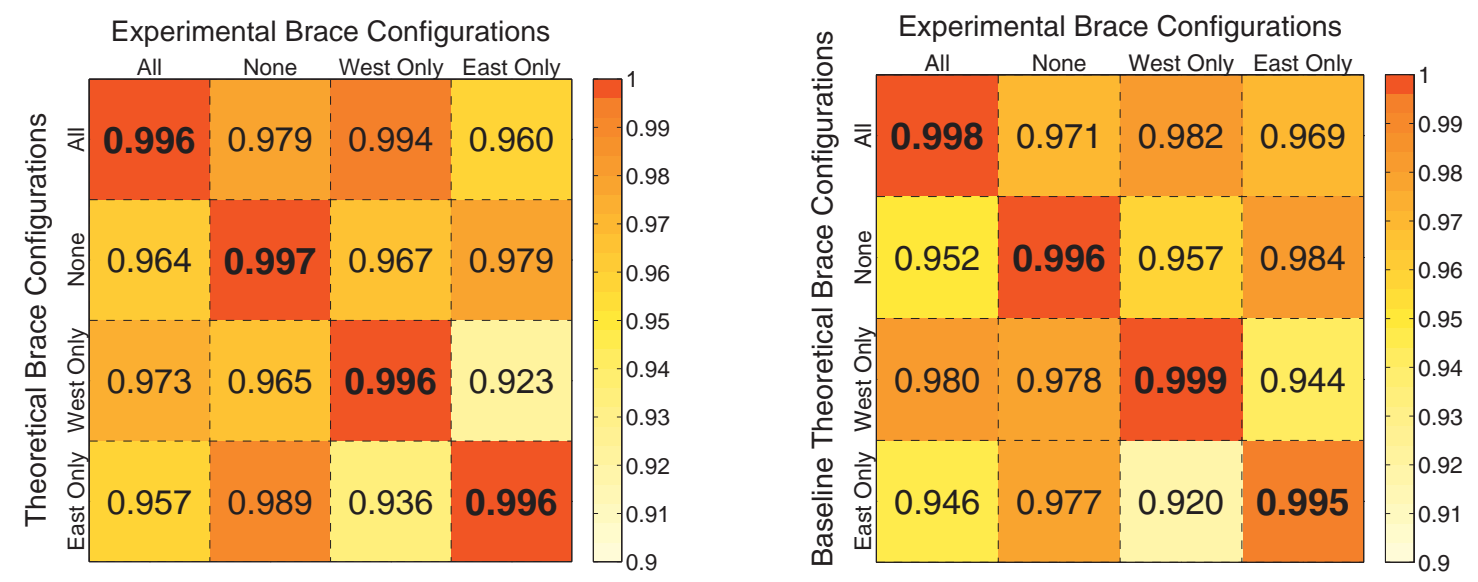

Figure 8-29: Non-Structural Theoretical MAC (left), Baseline MAC (right)

Additionally, this trend continues for every brace configuration. Most of the off diagonal values for the Theoretical MAC are greater than the Experimental MAC. Despite trying to model the non-structural elements, and despite the visual inspection showing more promising results, the MAC values yielded when trying to incorporate the significant non-structural elements did not yield better values. This could be for several reasons, but most noticeably, the stiffness of the additional elements is not well known, and greatly varied. These elements were approximated with a timber shear wall. Additionally, there is little room for improvement compared to the baseline. Without a proper method to quantitatively compare values, it is difficult to access minor changes in a MAC.

\subsubsection{Non-Structural Conclusion}

Visually, the roof behavior has been accurately captured by adding non-structural elements. The Theoretical MAC however did not show better values. In fact, it showed slightly lower values. Regardless, very high correlations were found on the diagonal and the MAC was successful in predicting brace configurations. For the Bridge House, the non-structural elements do not need to be modeled as they do not greatly contribute to the behavior. 


\subsubsection{Conclusion to Parametric Studies}

A summary of the results of the parametric studies are shown below in Figure 8-30.

Green checkmarks indicate all four brace configurations were correctly identified, red x-marks indicate that at least one brace configuration was incorrectly identified.

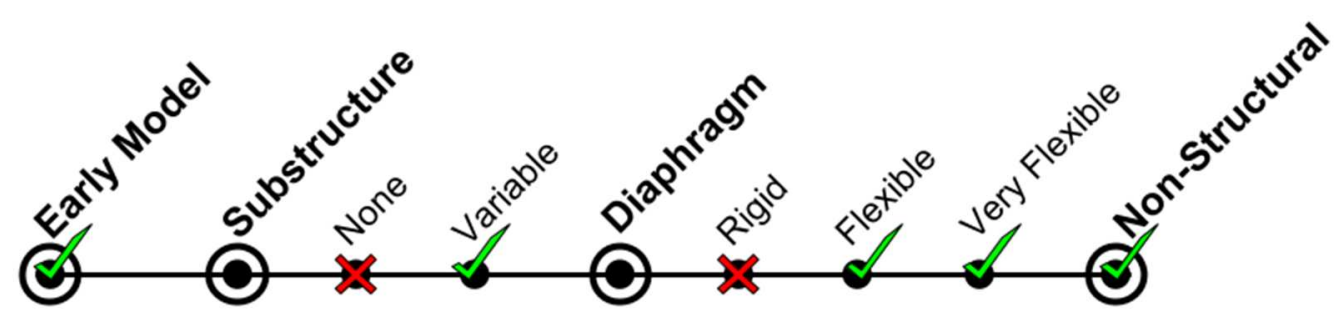

Figure 8-30: Parametric study results

Several important discoveries were made throughout these parametric studies. These main observations for the Bridge House are outlined below.

- Blind damage detection is possible using the Modal Assurance Criterion. The Modal Assurance Criterion is robust for a wide variety of modeling assumptions.

- The stiffness identification methods used are reliable and can greatly improve the model greatly.

- When using a MAC to identify damage, low off-diagonal values should not be used as a metric to determine lack of correlation. A poor model can show strong correlations on the diagonal and very low values on the off-diagonal. The best metric when using MACs is to look for extremely high correlations on the diagonal.

- Visually inspecting mode shapes gives insight to MAC results and mode shape behavior. 


\subsection{Procedural Changes}

\subsubsection{Overview}

Using the Baseline Model, changes to the experimental analysis procedure were made to determine how much experimental data is necessary to establish significant correlations between theoretical models and experimental data. Specifically, experimental data sets that have combinations of fewer degrees of freedom, along with added rotational degrees of freedom will be examined. For each study, the Experimental MAC has to be re-established as the amount of experimental data has changed and the mass matrix used for analysis has changed as well. The substructure and roof diaphragm stiffness values will not be recalculated with the revised degrees-of-freedom. This study focuses on the ability to detect damage with different data rather than system identification.

First, the Experimental MAC with revised DOF will replace the Experimental MAC with fourteen DOF. This will establish if the revised degrees-of-freedom has smaller values on the off-diagonal and, in turn, indicates larger differences between brace configurations.

Second, the revised Baseline MAC with the new set of DOF will be compared to the new Experimental MAC that also has the same set of DOF. This will show if the revised DOF are still able to identify brace configurations.

Table 8-2: Summary of procedural changes

\begin{tabular}{|c|c|c|}
\hline Step & Question & Comparison \\
\hline $\begin{array}{c}\text { Experimental } \\
\text { Comparison }\end{array}$ & Do fewer DOF make brace & Experimental MAC (Revised DOF) \\
configurations look more similar? & vs Experimental MAC (14 DOF) \\
\hline $\begin{array}{c}\text { Theoretical } \\
\text { Comparison }\end{array}$ & $\begin{array}{c}\text { Can brace configurations be } \\
\text { identified with fewer DOF? }\end{array}$ & $\begin{array}{c}\text { Theoretical MAC (Revised DOF) vs } \\
\text { Baseline MAC (14 DOF) }\end{array}$ \\
\hline
\end{tabular}

The goal of the following section is to show if fewer DOF are just as robust, if not more robust, in determining the brace configuration. If successful, it will show that fewer DOF are just as capable of predicting damage. 


\subsubsection{Nine DOF (7 Translational Roof, 1 Translational Floor, 1 Rotational Floor)}

\subsubsection{Experimental Comparison}

At the beginning of the research, it was verified that the floor of the Bridge House acts as a rigid diaphragm. In lieu of translation and rotation DOF, translational degrees of freedom were favored due to simplified calculations. To investigate if this assumption yields similar results, a parametric study was done that replaces the seven translational DOF on the floor, with one translational and one rotational. The selected DOF are shown in Figure 8-31.
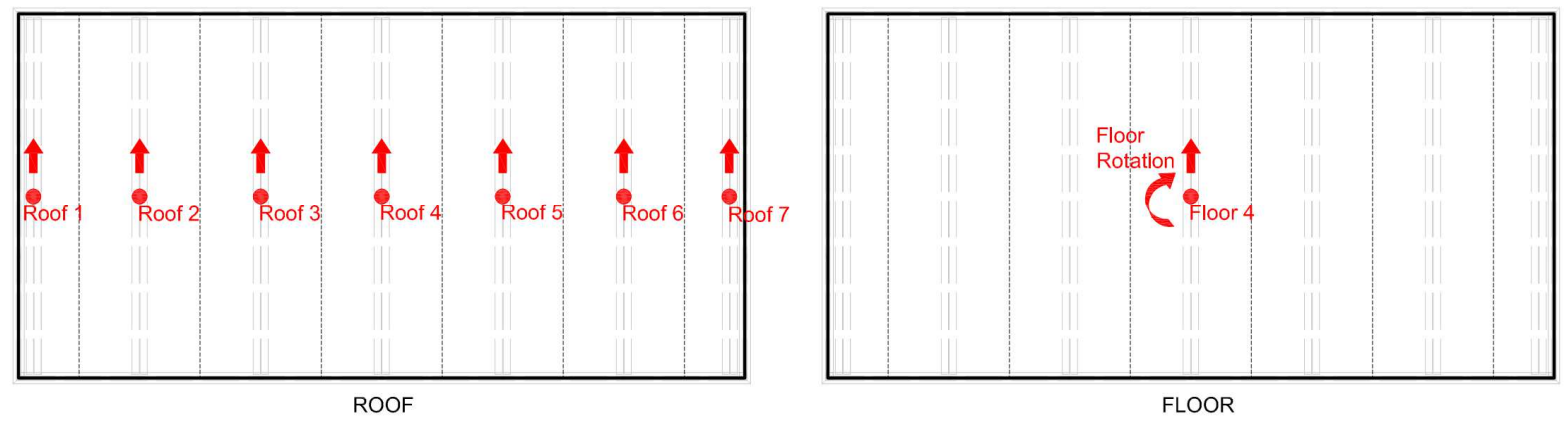

Figure 8-31: 9 DOF system

When comparing the revised 9 DOF Experimental MAC to the previous 14 DOF Experimental MAC, it's found that the values are identical. When substituting translational DOF with a single translational and single rotational DOF, there is no difference in the MACs and there is no difference in how the rigid-body behavior is identified. The 9 DOF Experimental MAC and 14 DOF Experimental MAC are shown in Figure 8-32. 

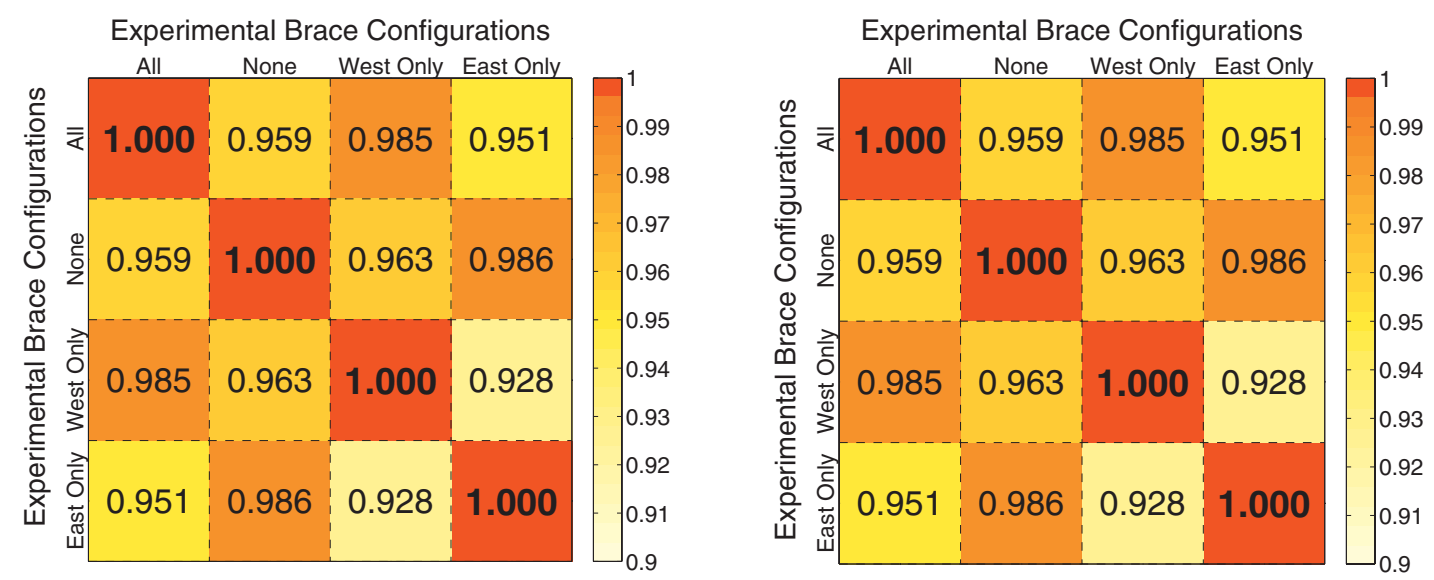

Figure 8-32: Experimental MAC - 9 DOF (left), Experimental MAC (right) - 14 DOF

\subsubsection{Theoretical Comparison}

The results in Figure 8-33 shows almost identical results when comparing the 9 DOF Theoretical MAC against the 14 DOF Theoretical MAC. A few values are slightly different, but for practicality and application, they are the same. This is further evidence that using reduced DOF to capture rigid body behavior is acceptable.
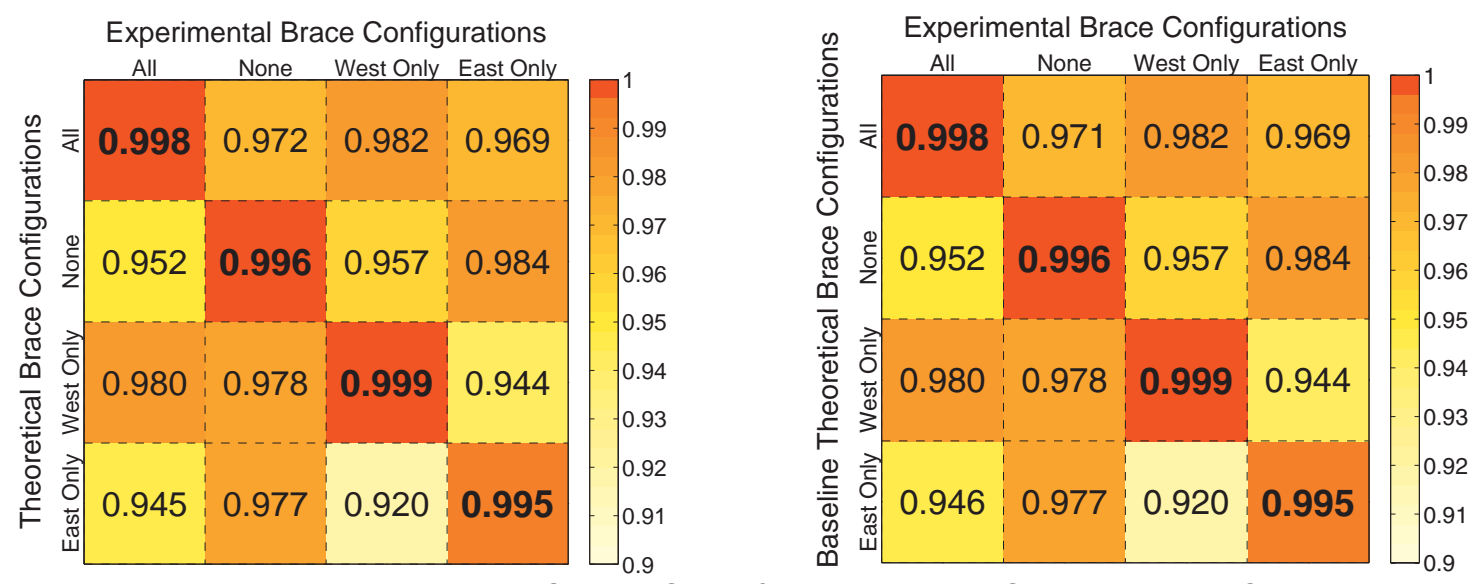

Figure 8-33: Baseline MAC - 9 DOF (left), Baseline MAC (right) - 14 DOF 


\subsubsection{Five DOF (3 Translational Roof, 1 Translational Floor, 1 Rotational Floor)}

\subsubsection{Experimental Comparison}

Seven roof accelerometers were originally used to capture roof behavior. It was hypothesized that this quantity of accelerometers were unnecessary. To test this hypothesis, an analysis with five DOF was done with three in the roof and two in the floor. The chosen DOF are shown below.
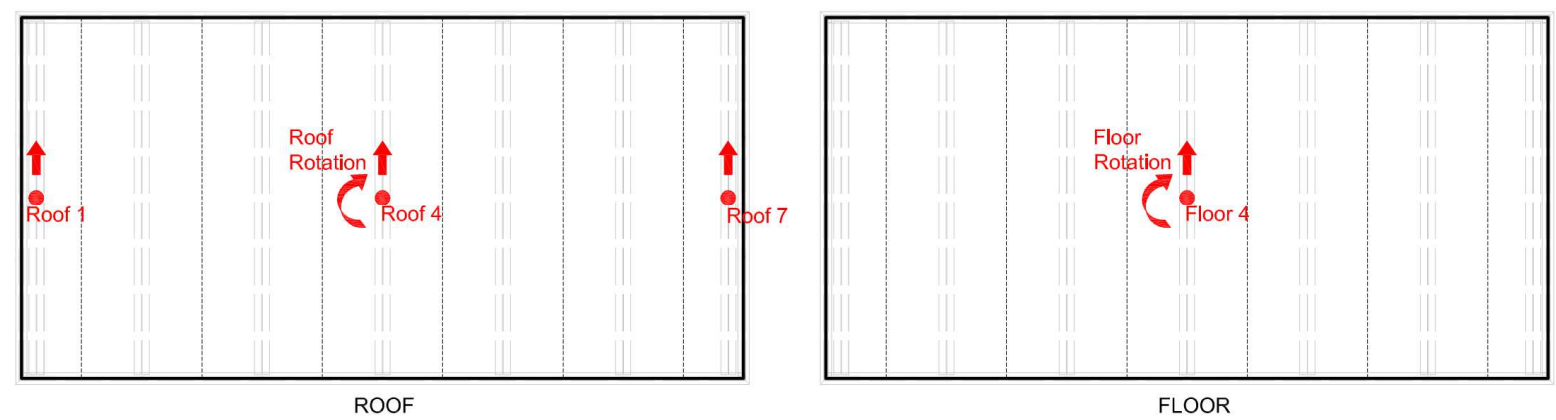

Figure 8-34: 5 DOF system

The theory behind this analysis is that the flexible roof behavior can be captured with only three accelerometers instead of the seven used during testing. The 5 DOF Experimental MAC and the 14 DOF MAC are shown below in Figure 8-35. While there are slight differences between the two MACs, they are overwhelming similar.
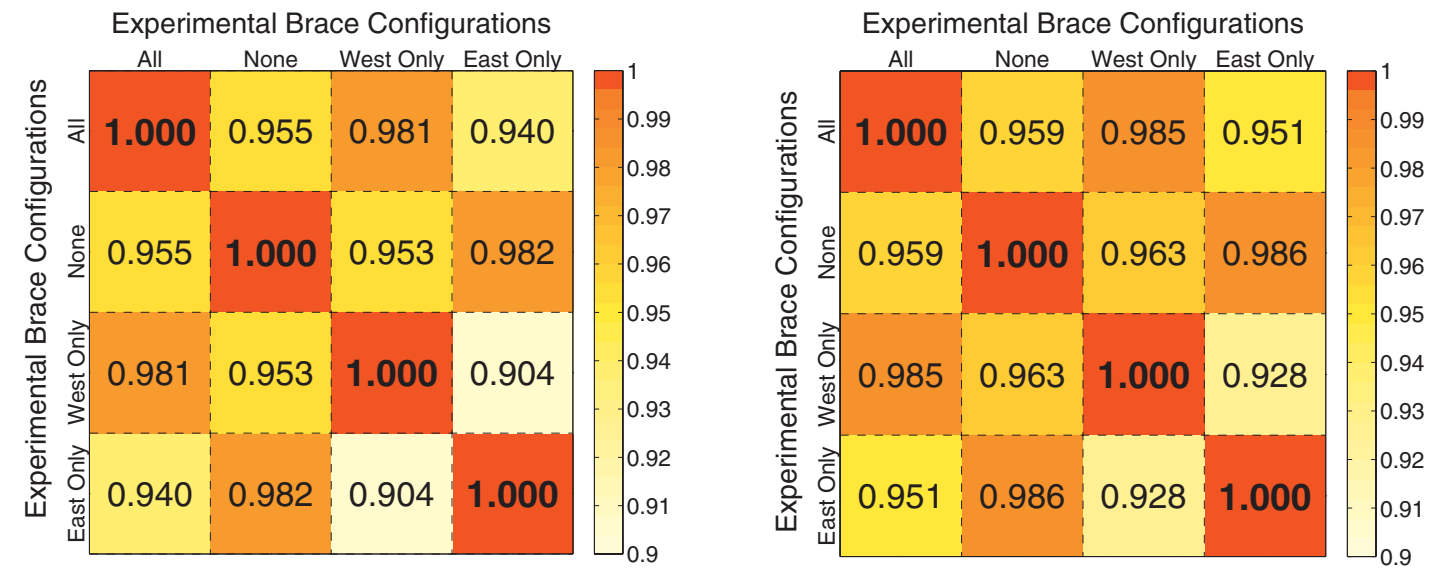

Figure 8-35: Experimental MAC - 5 DOF (left), Experimental MAC (right) - 14 DOF 
Experimentally, there is very little difference in using three accelerometers to identify the roof behavior compared to using seven accelerometers.

\subsubsection{Theoretical Comparison}

Figure 8-36 shows the Experimental Results and Theoretical Results with 5 DOF. Visually, the mode shapes are still identifiable, with the exception of the No Braces configuration. This is due to the additional stiffness of the non-structural elements on the East side of the structure.
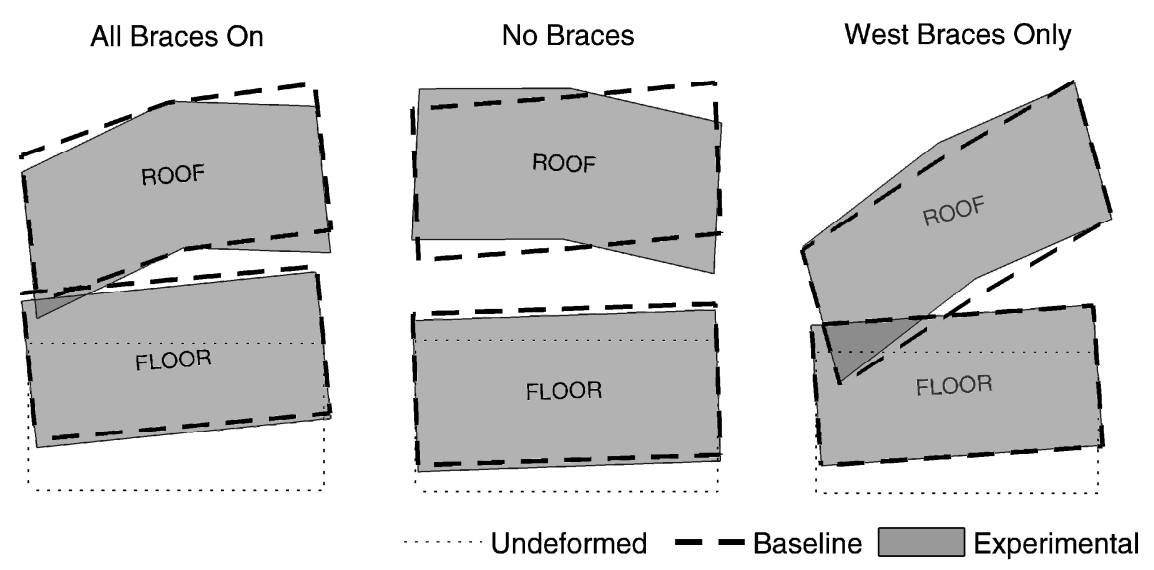

East Braces Only

Figure 8-36: 5 DOF mode shapes

The 5 DOF Baseline MAC has the highest values on the diagonal and successfully identified all the brace configurations. The 5 DOF Baseline MAC is also similar to the 14 DOF Baseline Theoretical MAC. The off-diagonal values in the 5 DOF Baseline MAC are typically slightly lower than those in the 5 DOF Theoretical MAC. Previous discussion has addressed the phenomena of lower off-diagonal values. The MACs for these results are shown in Figure 8-37. 

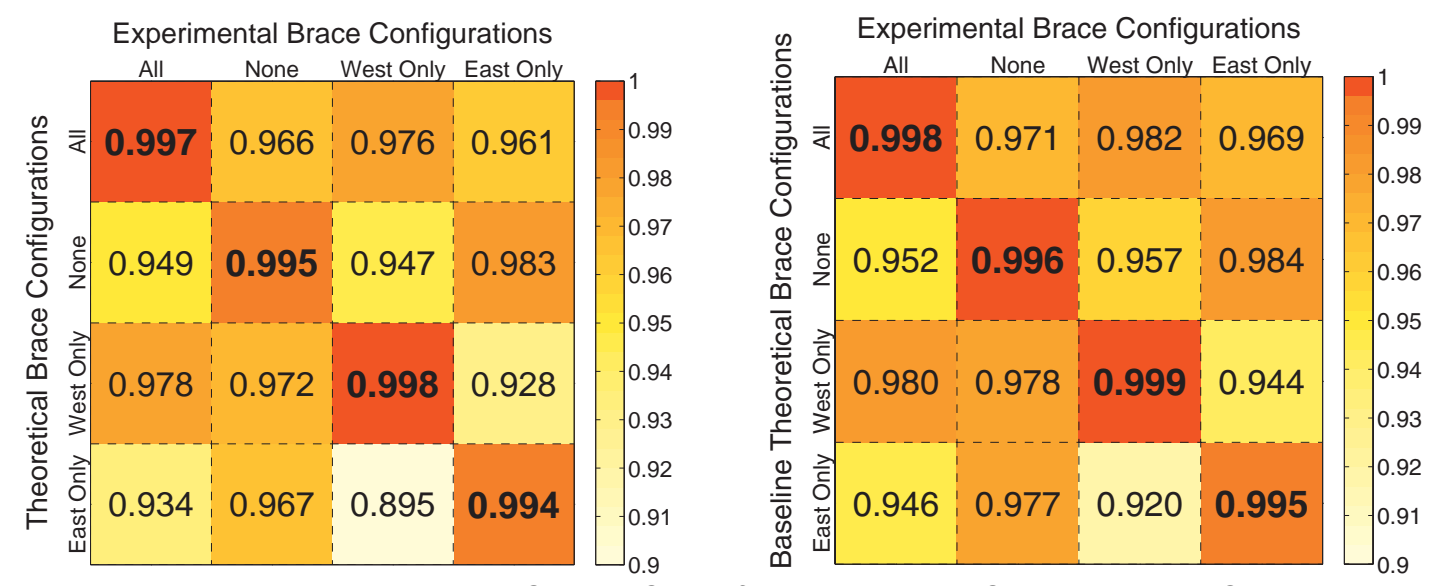

Figure 8-37: Baseline MAC - 5 DOF (left), Baseline MAC (right) - 14 DOF

Having less DOF appears to slightly lower off-diagonal values for the $5 \mathrm{DOF}$

Experimental MAC, but only slightly and insignificantly. Using three DOF in lieu of many DOF to capture flexible diaphragm behavior is acceptable and provides equally as robust results.

\subsubsection{Four DOF (2 Translational + 2 Rotational)}

\subsubsection{Experimental Comparison}

The simplest DOF freedom reduction is treating the roof and the floor of the Bridge House as rigid body members. Typically, rigid body behavior needs three degrees-of-freedom. Because there is minimal horizontal behavior, it was not recorded and will be ignored. The reduced DOF are shown below.
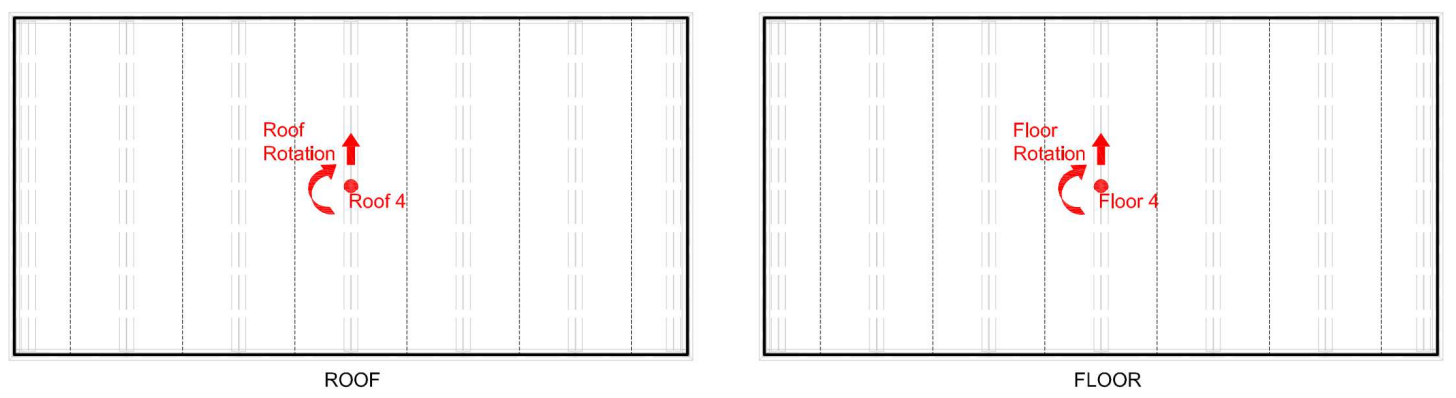

Figure 8-38: Roof DOF (left), Floor DOF (right) 
Compared to the 14 DOF Experimental MAC, the revised 4 DOF Experimental MAC yielded much lower off-diagonal values for every brace configuration. Even though some values were much lower, the off-diagonal values from the 14 DOF Experimental MAC that had high correlations were only slightly lower in the 4 DOF Experimental MAC. This indicates that the chosen 4 DOF shows less correlation between different brace configurations.
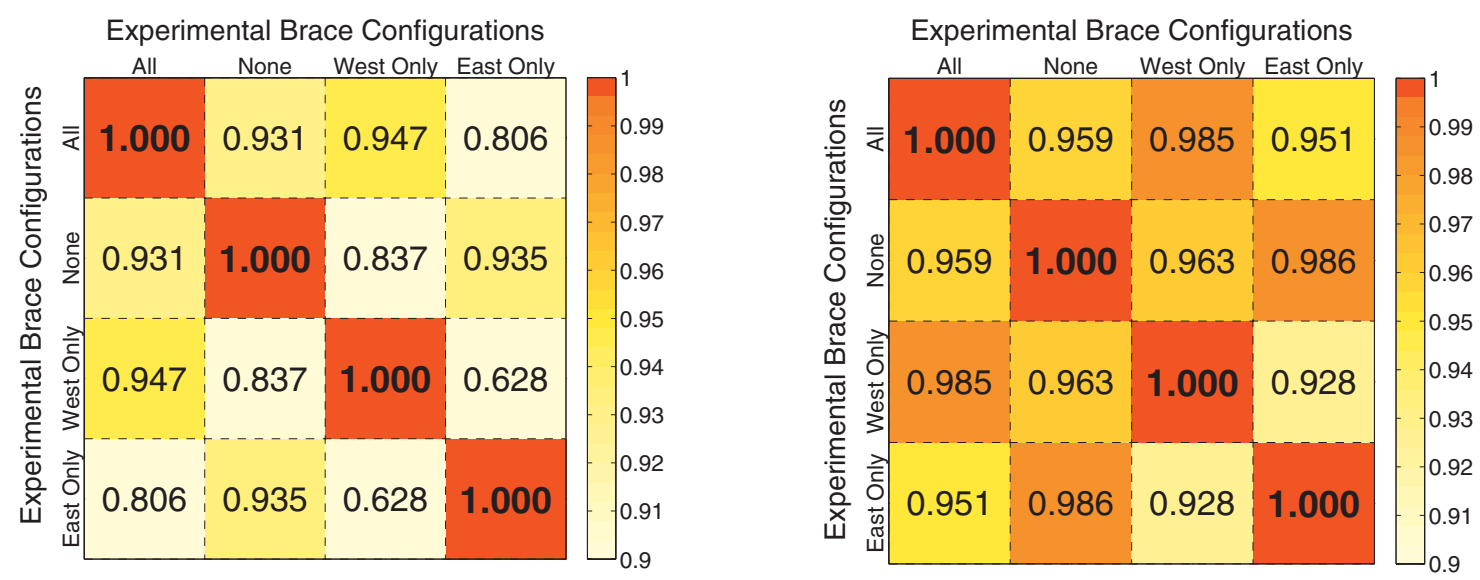

Figure 8-39: Experimental MAC - 4 DOF (left), Experimental MAC (right) - 14 DOF

While this seems to indicate that the chosen 4 DOF is "better" than the 14 DOF, this statement cannot be justified. It appears, though, that the chosen 4 DOF has the potential to have more leniency than the 14 DOF.

\subsubsection{Theoretical Comparison}

When simplifying the testing to $4 \mathrm{DOF}$, the roof is modeled as rigid and all the flexible diaphragm behavior is lost. A visual inspection shows the experimental results look much like the theoretical results. 


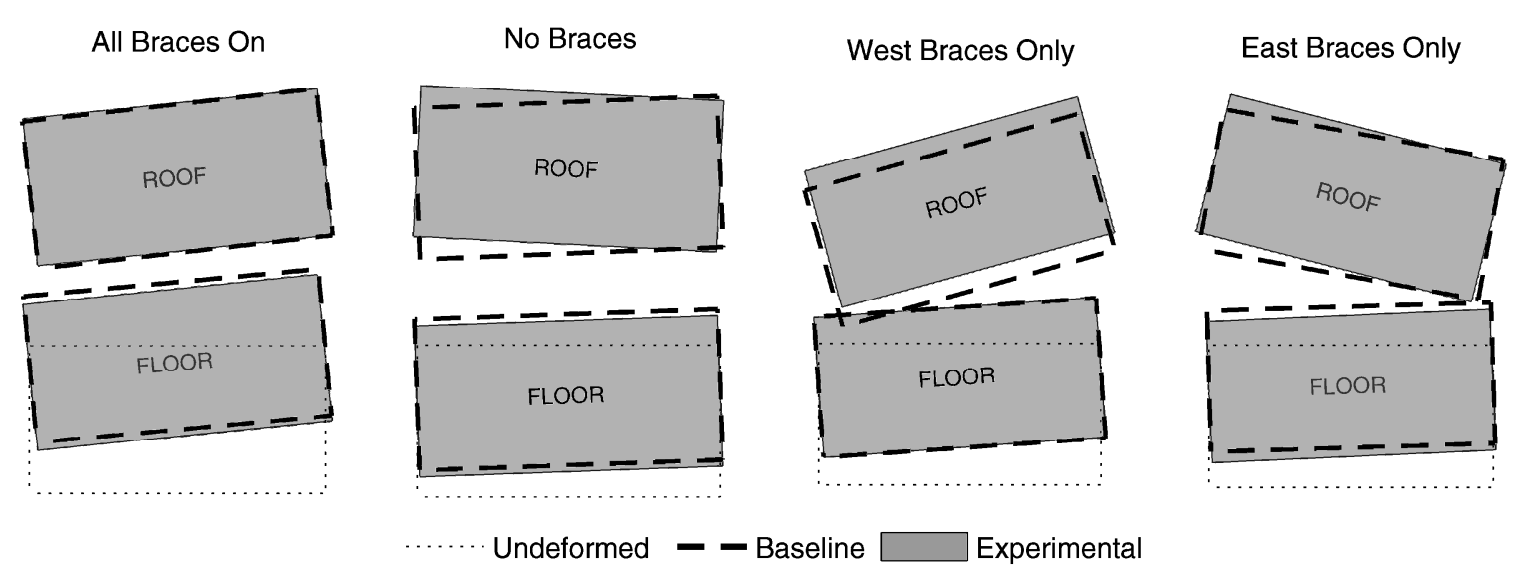

Figure 8-40: 4 DOF mode shapes

As with all the other scenarios, the No Braces configuration is the hardest to distinguish due to the stiff non-structural on the East side. The 4 DOF Baseline MAC was able to produce the highest values on the diagonal; the Baseline Model was successful in predicting the damaged brace configurations. In many cases, the off-diagonal values of the 4 DOF Baseline $M A C$ were lower than the off-diagonal values of the 4 DOF Experimental MAC.
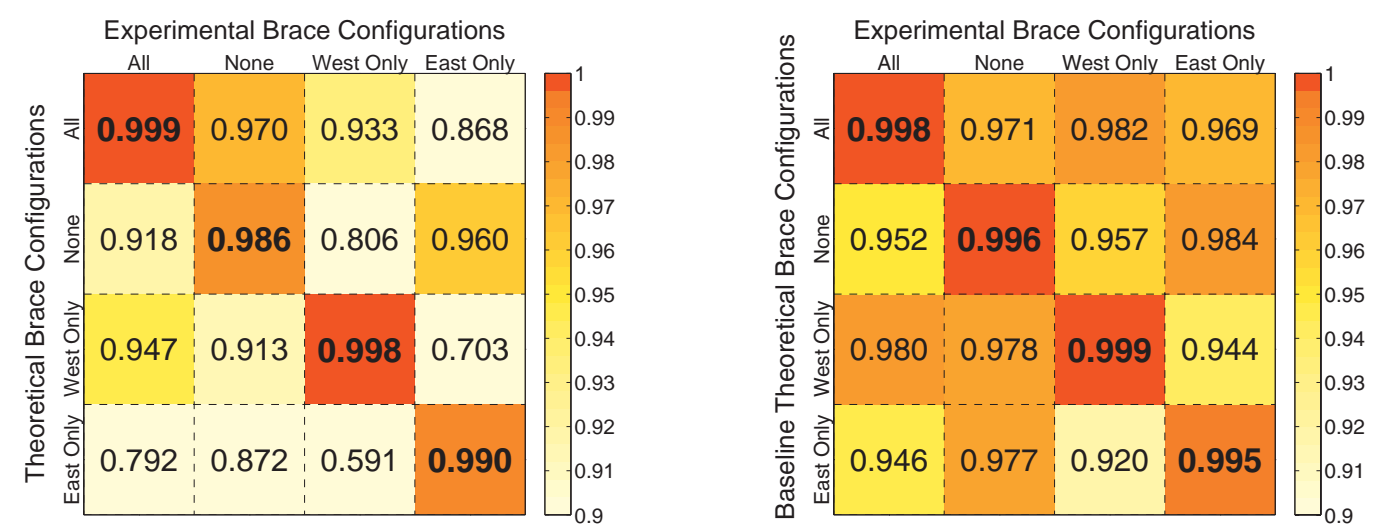

Figure 8-41: Baseline MAC - 4 DOF (left), Baseline MAC (right) - 14 DOF

It is impossible to have the Baseline MAC produce lower off-diagonal values and to consider that to be better results; getting a Baseline MAC that yields less correlation than a real world example is not possible. As per previous studies, it appears the best indicator when using the MAC is a strong correlation on the diagonals. It is also evidence that more accelerometers 
are able to stabilize the data; an exaggeration due to model variances is amplified when there are fewer DOF.

\subsubsection{Conclusion of Procedural Changes}

It has been shown a large number of accelerometers yield little advantage. It was found:

- In a rigid body that moves predominately translationally, a rotational and single translational DOF captures behavior equally well when compared to many translational accelerometer locations. There is negligible difference in analysis.

- Flexible behavior of a diaphragm should not be captured with one translation DOF and one rotational DOF. However, a rigid assumption may possible, but should be discouraged if possible.

- Three accelerometers are sufficient in mapping flexible behavior. More accelerometer locations yield no additional information for damage detection purposes. 


\subsection{CONCLUSION}

\subsection{System Identification}

Using first mode experimental data, the system identification methods outlined in this thesis to identify structure stiffness succeeded in capturing the behavior of the structure. The methods developed in a lab environment (Raney et al., 2015) translated well for the Experimental Dynamics Field Laboratory, most commonly referred to in this thesis as the Bridge House. These results improved model accuracy. Variance was found in both the substructure properties and the diaphragm stiffness. This variance was negligible and did not impact the ability to correctly identify the brace configurations.

\subsection{Damage Detection}

\subsubsection{Modal Assurance Criterion}

The use of the Modal Assurance Criterion to detect damage was successful. The Baseline Model results were used to accurately identify all four tested brace configurations to the corresponding experimental brace configuration. The Experimental MAC indicated that every experimental brace configuration at the Bridge House was similar to one another. Even with these strong correlations, the Modal Assurance Criterion was successful.

Previously, the use of the Modal Assurance Criterion to detect structural damage relied on two assumptions. The first assumption being that, in every column of the MAC, the highest value should also be on the diagonal. The second assumption asserted that the better results should yield a larger number on the diagonal and smaller numbers on the off-diagonals. This research indicated that the last assumption is not valid. A model that clearly does not capture the behavior of the Bridge House produced low off-diagonal values while still creating reasonably high diagonal values. This assumption should be investigated in further research. 
For this research, the last assumption was revised to ignore low off-diagonal values as an indicator of lack-of-correlation.

\subsubsection{Model Accuracy}

The parametric studies showed a wide variety of models are capable of correctly predicting brace configurations. Specifically, the roof diaphragm stiffness could be modeled with wide bounds and the MAC values displayed strong correlations between experimental brace configurations and corresponding theoretical brace configurations. Likewise, an early, undeveloped model was able to predict damage. This model had rough estimates of the substructure and roof diaphragm behavior.

\subsubsection{Data Collection}

The quantity of DOF used for measurement in this research exceeded the minimum amount needed for successful results. When representing a flexible diaphragm as a rigid body with two DOF, the results were inconclusive. Flexible diaphragm behavior should be accounted for with additional DOF. However, more than 3 DOF did not increase the accuracy on a flexible diaphragm.

\subsection{Suggestions for Future Research}

\subsubsection{Bridge House}

For future research on the Bridge House, it is recommended that several studies be conducted. First, it is suggested that higher modes be incorporated into the research. Software, equipment, and time constraints prohibited the data acquisition of higher modes for this research. Previous research (Gerbo, 2014) has indicated that including higher modes could provide further validity for the use of the Modal Assurance Criterion to identify damage. 
It is also suggested that a refinement to the brace states be conducted. For this research, four brace configurations were tested with binary states, either engaged or disengaged. These states correlated to an intact brace, or a fully ruptured brace, respectively. While braces do fully rupture and complete stiffness is lost, they are more likely to buckle, partially rupture, or locally buckle. Each one of these scenarios would result in a stiffness reduction. It is recommended that these scenarios be examined in order to create a scenario more analogous to the real world.

Lastly, additional tools could be established to help with damage detection in the future. For this research, mode shapes were normalized and magnitudes of the experimental accelerations were disregarded. It is possible that additional information could be gained from developing a method that accounts for the magnitude of the accelerations.

\subsubsection{Suggestions for Application}

The methods outlined in this research have the most practicality when they are streamlined and applied to a large building. For a mobile system, solutions for the workflow must be turnkey. System identification and data acquisition is not always a straight forward procedure. The more that can be done to quickly and correctly identify the modes in the field, the more advantageous this method becomes.

Additionally, there is potential for integration with existing building health monitoring systems. A large earthquake can displace tenants for weeks or months at a time. Today, many companies with large buildings or critical systems are retaining engineers in the case a seismic event occurs. Building monitoring systems are increasing in popularity and having tools that can accurately assess damaged locations is advantageous. The cost of having permanent equipment installed has the potential to reduce revenue lost due to downtime. 


\section{BIBLIOGRAPHY}

Archer G.C., McDaniel C.C. "An Experimental Study Of Damage Detection Using Removable Braces." 10th U.S. National Conference on Earthquake Engineering, Earthquake Engineering Research Institute, 2014.

Allemang R. J. "The Modal Assurance Criterion (MAC): Twenty Years of Use and Abuse." Sound and Vibration, 2003.

Ross, Ben. Ben + Jo Photo | Bridge House. N.p., n.d. Web. 3 Feb. 2016. <benandjophoto.com>.

Chopra, Anil K. Dynamics of Structures: Theory and Applications to Earthquake Engineering. Boston: Prentice Hall, 2012. Print.

CSI Analysis Reference Manual. (2013). Berkeley, California: Computers \& Structures Inc.

Davena, Ronald, et al. California Polytechnic State University, San Luis Obispo, 1966. San Luis Obispo: Cal Poly SLO, 1966. Print.

Garlow, Robert. "Bridge House Inquiry." Message to the author. 24 Jan. 2016. E-mail.

Gerbo, Evan Jamison. "Structural Damage Detection Utilizing Experimental Mode Shapes." Thesis. California Polytechnic University, San Luis Obispo, 2014. 31 July 2014. Web. <digitalcommons.calpoly.edu/theses/1247>.

McDaniel, C., Archer, G. Classroom-Based Forced-Vibration Testing, 15th World Conference on Earthquake Engineering (15th WCEE), Lisbon, Portugal, 2012.

Planas, Brian, et al. "Rehabilitation of the Bridge House". California Polytechnic State University, San Luis Obispo, 2011. San Luis Obispo: Cal Poly SLO, 2011. Print 
Ramos, Pablo D., Jr. "System Identification Of A Bridge-type Building Structure." Thesis. California Polytechnic University, San Luis Obispo, 2013. 18 June 2013. Web. <digitalcommons.calpoly.edu/theses/944>.

Raney, Joshua, et al. "Influence of Boundary Conditions on Building Behavior". 2015 ASEE Annual Conference and Exposition, Seattle, Washington, 2015, June. ASEE Conferences, 2015. https://peer.asee.org/24296 Internet. 09 Feb, 2016. 


\section{APPENDICES}

\section{A.1 Response Spectrum Analysis}

The following section outlines the theory of steady state response. Derivations and equations are summarized from Anil K. Chopra's Dynamics of Structures ( ${ }^{\text {th }}$ Edition).

\section{Steady-State Response}

The equation of motion for a single degree of freedom with viscous damping subjected to a sinusoidal force is:

$$
m \ddot{u}+c \dot{u}+k u=p_{0} \sin \omega t
$$

Equation $A-1$

The left side represents the internal forces of the system, the right side represents the external forces applied to the system. Dividing by $m$ yields:

$$
\ddot{u}+\frac{c}{m} \dot{u}+\frac{k}{m} u=\frac{p_{0}}{m} \sin \omega t
$$

Equation A-2

To simplify Equation A-2, relationships between the variables $k, m$, and $c$ must be defined. The following steps will establish relationships. The first relationship to establish is the relationship between stiffness and mass by using the natural frequency of the system. The natural frequency of a system is defined as:

$$
\omega_{n}=\sqrt{\frac{k}{m}}
$$

Squaring the natural frequency yields:

$$
\omega_{n}^{2}=\frac{k}{m}
$$

Equation A-4

The next relationship that will be established will relate the damping coefficient to the dynamic properties of the system. This derivation starts with the critical damping coefficient and 
the damping ratio. The critical damping coefficient is the value where the system does not oscillate beyond one cycle and is defined by:

$$
c_{c r}=2 m \omega_{n}
$$

Equation A-5

The damping ratio, or the ratio of damping to the critical damping, is represented by variable zeta:

$$
\zeta=\frac{c}{c_{c r}}
$$

Equation A-6

Substituting Equation A-5 into Equation A-6 yields:

$$
\zeta=\frac{c}{2 m \omega_{n}}
$$

Re-arranging Equation A-7 gives:

$$
\frac{c}{m}=2 \zeta \omega_{n}
$$

The previously established relationships will be substituted into the equation of motion. Substituting Equation A-4 and Equation A-8 into Equation A-2 yields:

$$
\ddot{u}+2 \zeta \omega_{n} \dot{u}+\omega_{n}^{2} u=\frac{p_{0}}{m} \sin \omega t
$$

Equation A-9

In this form, Equation A-9 has a particular (or steady state) solution of:

$$
u_{p}(t)=C \sin \omega t+D \cos \omega t
$$

The transient component of the solution to Equation A-9 is assumed to be negligible as the impact of the steady state response damps out after a short period of time. The increase in amplitude due to harmonic loading will be shown later. The first and second derivative of Equation $\mathrm{A}-10$ with respect to time are: 


$$
\begin{gathered}
\dot{u}_{p}(t)=\omega C \cos \omega t-\omega D \sin \omega t \\
\ddot{u}_{p}(t)=-\omega^{2} C \sin \omega t-\omega^{2} D \cos \omega t
\end{gathered}
$$

Equation A-11

Equation A-12

Substituting Equation A-11 and Equation A-12 into Equation A-9 yields:

$$
\left[\left(\omega_{n}^{2}-\omega^{2}\right) C-2 \zeta \omega_{n} D\right] \sin \omega t+\left[2 \zeta \omega_{n} \omega C+\left(\omega_{n}^{2}-\omega^{2}\right) D\right] \cos \omega t=\frac{p_{0}}{m} \sin \omega t \quad \text { Equation } A-13
$$

Extracting the sine and cosine for Equation A-13 yields two algebraic equations:

$$
\begin{gathered}
{\left[1-\left(\frac{\omega}{\omega_{n}}\right)^{2}\right] C-\left(2 \zeta \frac{\omega}{\omega_{n}}\right) D=\frac{p_{0}}{k}} \\
\left(2 \zeta \frac{\omega}{\omega_{n}}\right) C+\left[1-\left(\frac{\omega}{\omega_{n}}\right)^{2}\right] D=0
\end{gathered}
$$

Simultaneously solving Equation A-14 and Equation A-15 for coefficients $C$ and $D$ yields:

$$
\begin{aligned}
& C=\frac{p_{0}}{k} \frac{1-\left(\frac{\omega}{\omega_{n}}\right)^{2}}{\left[1-\left(\frac{\omega}{\omega_{n}}\right)^{2}\right]^{2}+\left[2 \zeta\left(\frac{\omega}{\omega_{n}}\right)\right]^{2}} \\
& D=\frac{p_{0}}{k} \frac{-2 \zeta\left(\frac{\omega}{\omega_{n}}\right)}{\left[1-\left(\frac{\omega}{\omega_{n}}\right)^{2}\right]^{2}+\left[2 \zeta\left(\frac{\omega}{\omega_{n}}\right)\right]^{2}}
\end{aligned}
$$

Equation A-16 and Equation A-17 are the coefficients for Equation A-10. The outlined proof is for steady state behavior. Traditionally, dynamic response would also have transient, homogenous behavior. This will be ignored as its contribution during steady state is small and irrelevant. It will be shown in the following sections that the response due to forced vibration is much larger. 


\section{Maximum Deformation}

The basis of system identification in modal testing relies on the ability to predict the maximum displacements from experimental data. To calculate the maximum displacements, the following formulation is used. The solution to Equation A-10 can be defined by the following trigometric identity.

$$
C \sin \alpha+D \cos \alpha=\sqrt{C^{2}+D^{2}} \cos \left(\alpha-\tan ^{-1}(D / C)\right)
$$

The right hand side of Equation A-8 can be simplified to the following terms:

$$
u_{p}(t)=u_{\max } \sin (\omega t-\phi)
$$

Where $u_{m a x}$ is the maximum dynamic displacement due to a sine vibration:

$$
u_{\max }=\sqrt{C^{2}+D^{2}}
$$

And where $\phi$, the phase lag:

$$
\phi=\tan ^{-1}(D / C)
$$

To solve for the maximum displacement, variables $D$ and $C$ from Equation A-16 and Equation $\mathrm{A}-17$ are substituted into Equation A-20:

$$
u_{\text {max }}=\sqrt{\left(\frac{p_{0}}{k} \frac{1-\left(\frac{\omega}{\omega_{n}}\right)^{2}}{\left[1-\left(\frac{\omega}{\omega_{n}}\right)^{2}\right]^{2}+\left[2 \zeta\left(\frac{\omega}{\omega_{n}}\right)^{2}\right]}\right)^{2}+\left(\frac{p_{0}}{k} \frac{-2 \zeta\left(\frac{\omega}{\omega_{n}}\right)}{\left[1-\left(\frac{\omega}{\omega_{n}}\right)^{2}\right]^{2}+\left[2 \zeta\left(\frac{\omega}{\omega_{n}}\right)^{2}\right]}\right)^{2}}
$$

Further simplification produces the following equations: 


$$
\begin{aligned}
& u_{\max }=\frac{p_{0}}{k} \sqrt{\left(\frac{1-\left(\frac{\omega}{\omega_{n}}\right)^{2}}{\left[1-\left(\frac{\omega}{\omega_{n}}\right)^{2}\right]^{2}+\left[2 \zeta\left(\frac{\omega}{\omega_{n}}\right)\right]^{2}}\right)^{2}+\left(\frac{-2 \zeta\left(\frac{\omega}{\omega_{n}}\right)}{\left[1-\left(\frac{\omega}{\omega_{n}}\right)^{2}\right]^{2}+\left[2 \zeta\left(\frac{\omega}{\omega_{n}}\right)\right]^{2}}\right)^{2}} \\
& u_{\max }=\frac{p_{0}}{k} \sqrt{\frac{\left[1-\left(\frac{\omega}{\omega_{n}}\right)^{2}\right]^{2}}{\left(\left[1-\left(\frac{\omega}{\omega_{n}}\right)^{2}\right]^{2}+\left[2 \zeta\left(\frac{\omega}{\omega_{n}}\right)\right]^{2}\right)^{2}}+\frac{\left[2 \zeta\left(\frac{\omega}{\omega_{n}}\right)\right]^{2}}{\left(\left[1-\left(\frac{\omega}{\omega_{n}}\right)^{2}\right]^{2}+\left[2 \zeta\left(\frac{\omega}{\omega_{n}}\right)\right]^{2}\right)^{2}}} \\
& u_{\max }=\frac{p_{0}}{k} \sqrt{\frac{\left[1-\left(\frac{\omega}{\omega_{n}}\right)^{2}\right]^{2}+\left[2 \zeta\left(\frac{\omega}{\omega_{n}}\right)\right]^{2}}{\left(\left[1-\left(\frac{\omega}{\omega_{n}}\right)^{2}\right]^{2}+\left[2 \zeta\left(\frac{\omega}{\omega_{n}}\right)\right]^{2}\right)^{2}}} \\
& u_{\max }=\frac{p_{0}}{k} \sqrt{\frac{1}{\left[1-\left(\frac{\omega}{\omega_{n}}\right)^{2}\right]^{2}+\left[2 \zeta\left(\frac{\omega}{\omega_{n}}\right)\right]^{2}}} \\
& u_{\max }=\frac{p_{0}}{k} \frac{1}{\sqrt{\left[1-\left(\frac{\omega}{\omega_{n}}\right)^{2}\right]^{2}+\left[2 \zeta\left(\frac{\omega}{\omega_{n}}\right)\right]^{2}}}
\end{aligned}
$$

It is important to note that the sine term from Equation A-19 has been disregarded as the only property being examined is the maximum amplification; this occurs when the sine term reaches its upper bound of one.

\section{Displacement Response Amplification Factor, Rd}

The displacement response amplification factor, $R_{d}$, is a ratio that compares the static deformations to the dynamic deformations of similar systems. The displacement response amplification factor is as follows: 


$$
R_{d}=\frac{u_{\max }}{\left(u_{s t}\right)_{0}}
$$

Equation A-24

Where $\left(u_{s t}\right)_{0}$ the deformation due the applied force, and $p_{0}$ is the amplitude of the applied force.

$$
\left(u_{s t}\right)_{0}=\frac{p_{0}}{k}
$$

Substituting Equation A-23 and Equation A-25 into Equation A-24 yields the following equation at steady state:

$$
R_{d}=\frac{\frac{p_{0}}{k} \frac{1}{\sqrt{\left[1-\left(\frac{\omega}{\omega_{n}}\right)^{2}\right]^{2}+\left[2 \zeta\left(\frac{\omega}{\omega_{n}}\right)\right]^{2}}}}{\frac{p_{0}}{k}}
$$

Which simplifies to:

$$
R_{d}=\frac{1}{\sqrt{\left[1-\left(\frac{\omega}{\omega_{n}}\right)^{2}\right]^{2}+\left[2 \zeta\left(\frac{\omega}{\omega_{n}}\right)\right]^{2}}}
$$

Plotting Equation A-27 for a variety of damping ratios of an excited system is show in Figure A-1. 


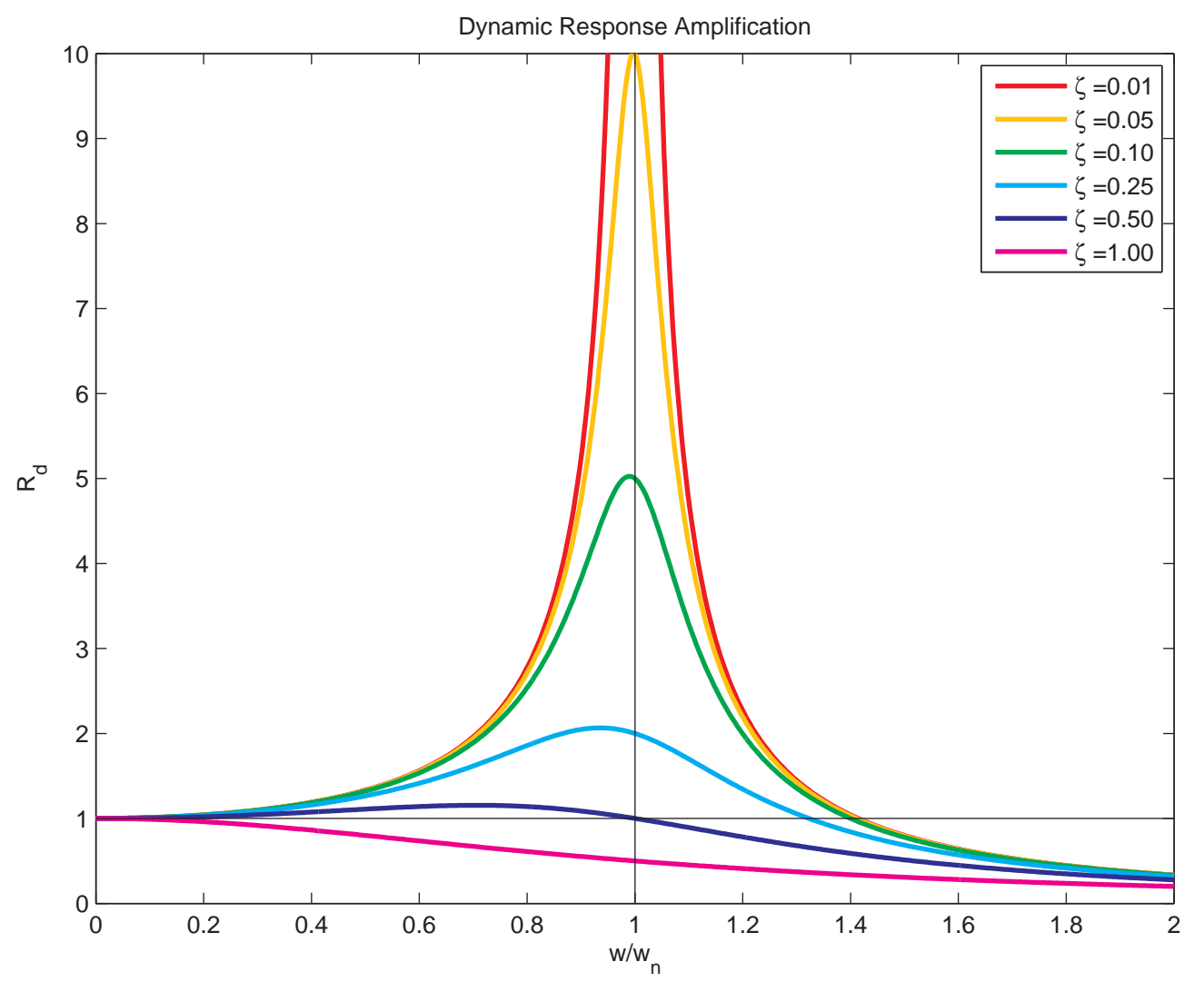

Figure A-1: Dynamic Response Displacement Amplification

Increasing damping for a system will reduce the displacements. For low dampened systems, (when $\zeta$ is approximately less than 10 percent), the maximum displacements occur when the system is excited at its natural frequency (when $w / w_{n}=1$ ). For systems with large damping, the maximum displacements will not occur at $w / w_{n}=1$. For non-damping building systems, zeta is typically well below 10 percent. Therefore, for the scope of this research, it shall always be assumed that the maximum displacement occurs when $w / w_{n}=1$. By exciting a building at a variety of frequencies, a response amplification plot as shown in Figure A-1 can be composed. 


\section{Phase Angle}

When calculating the maximum deformations at a steady state response, the phase of the system is ignored. The maximum displacement, $u_{\max }$, is time independent; the phase angle only accounts for the delay of the system when responding to the forcing function. Additionally, the impact that the phase angle has on the response is small. For reference, the steady state solution, the trigometric identities, and the simplification to the equation of motion is shown below.

$C \sin \alpha+D \cos \alpha=\sqrt{C^{2}+D^{2}} \cos \left(\alpha-\tan ^{-1}(D / C)\right)=u_{\max } \sin (\omega t-\phi)$

For reference, the phase angle from Equation A-21 is shown below.

$$
\phi=\tan ^{-1}(D / C)
$$

Substitution of terms $C$ and $D$ from Equation $A-16$ and Equation $A-17$, along with simplification, yields the phase angle in terms of the frequency ratio and damping coefficient.

$$
\phi=\tan ^{-1}\left(\frac{-2 \zeta\left(\omega / \omega_{n}\right)}{1-\left(\omega / \omega_{n}\right)^{2}}\right)
$$

Plotting the phase angle shows the delay of the structure response with respect to an external sinusoidal force with varying frequency. The phase angle is the difference in peaks of the forcing function and the building response. When the frequency ratio is one, the peaks of the dynamic force occur at the same time that the displacement peaks at the building. As the frequency ratio drifts from one, the peaks of the dynamic force and the response of the building drift apart. 


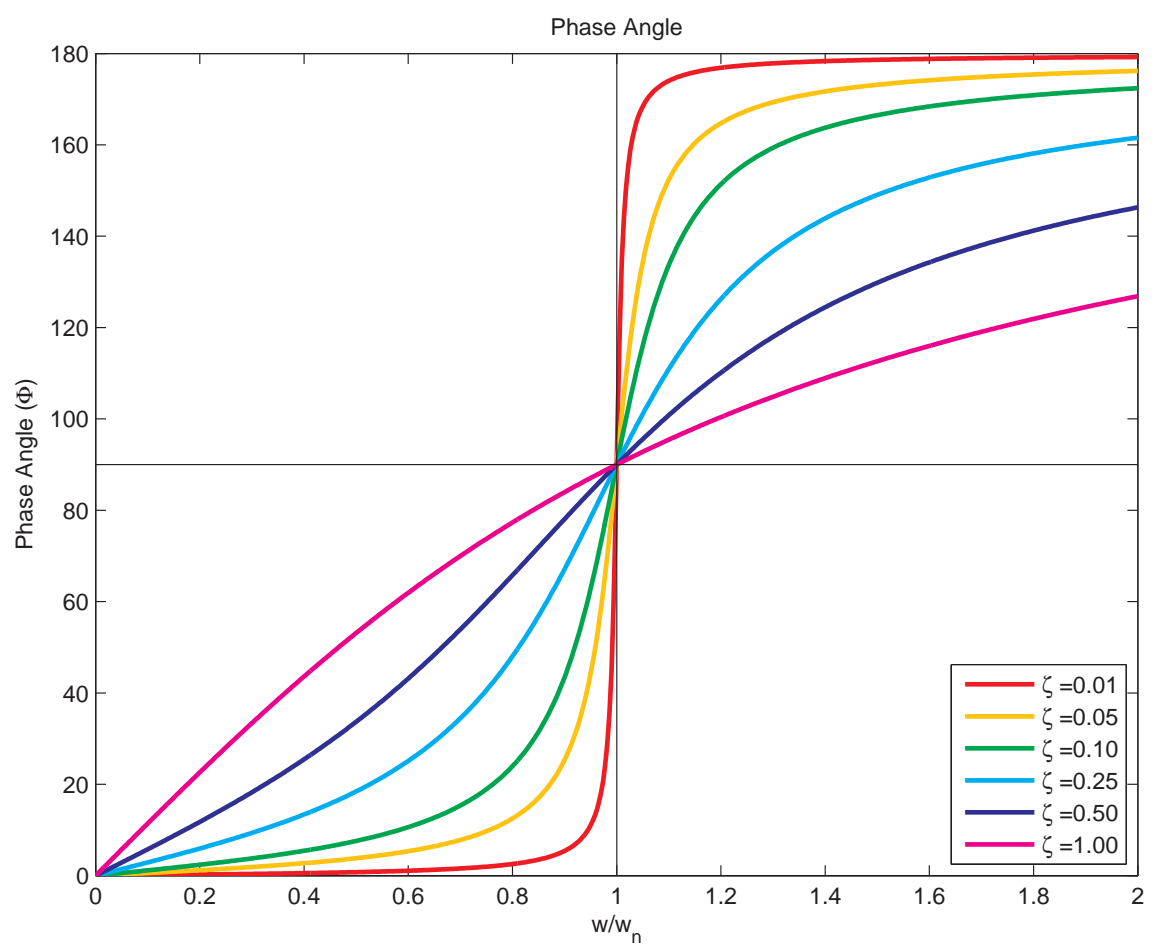

Figure A-2: Phase Angle

The phase angle gives important information to building response when not excited at its natural frequency. The phase angle is needed to predict the total behavior in the time-domain. In the frequency response domain, the importance of phase angle becomes less essential. The analysis used in this thesis will not focus on phase angle.

\section{Damping}

To experimentally calculate damping ratios, two methods can be utilized. One method utilizes the transient response properties due to the decay of motion. This method typically involves enforcing a displacement in the building, releasing the displacement, and recording the decay of the vibrations. By recording the time history, damping can be calculated. Because of the size and terrain limitations of the Bridge House, this method cannot be utilized. A second method called the Half-Power Bandwidth allows for the calculation of the damping from a displacement response amplification curve. 


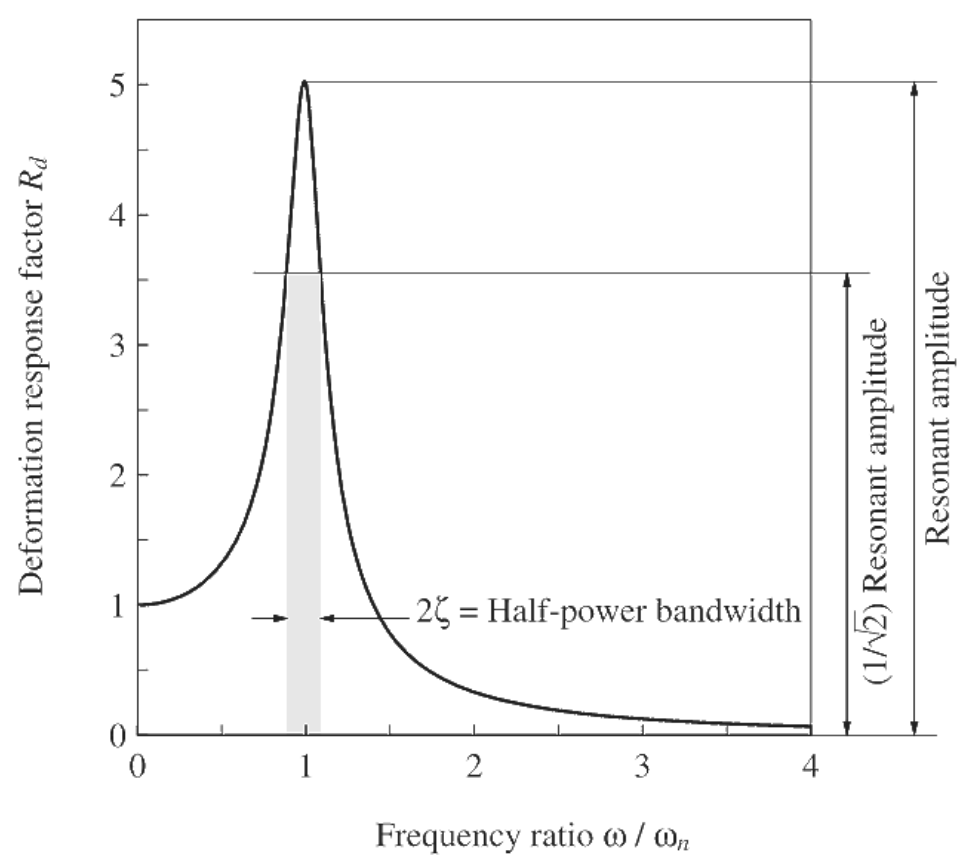

Figure A-3: Damping calculation (Chopra)

The half-power bandwidth method allows the calculation of the damping from the displacement frequency response curve. Simplification and substitution yields the following

$$
\zeta=\frac{f_{b}-f_{a}}{2 f_{n}}
$$

Where $f_{b}-f_{a}$ is defined as the bandwidth and $f_{n}$ is the natural frequency. In lieu of frequencies, periods may be used to calculate the damping ratio. 


\section{A.2 Raw Data}

All values from testing done on April 22, 2015.

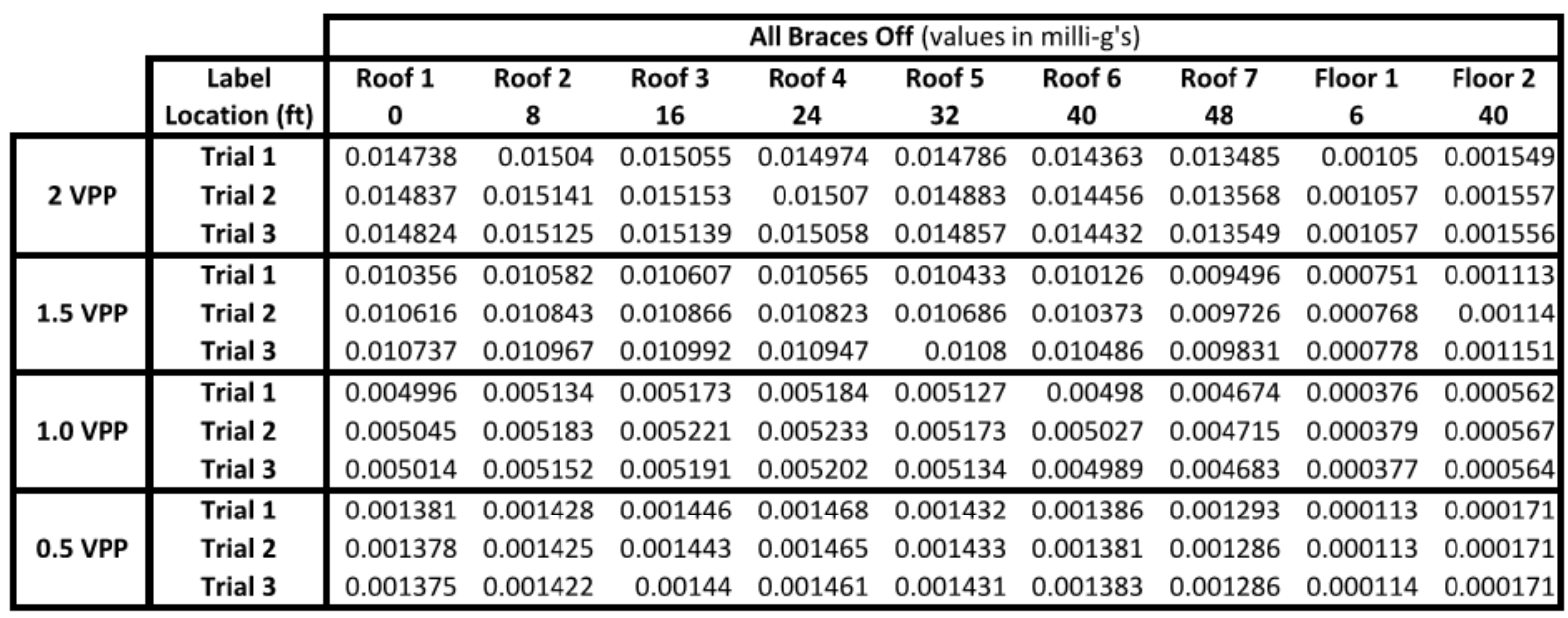

\begin{tabular}{|c|c|c|c|c|c|c|c|c|c|c|}
\hline & & \multicolumn{9}{|c|}{ All Braces On (values in milli-g's) } \\
\hline & $\begin{array}{c}\text { Label } \\
\text { Location (ft) }\end{array}$ & $\begin{array}{c}\text { Roof } 1 \\
0\end{array}$ & $\begin{array}{c}\text { Roof } 2 \\
8\end{array}$ & $\begin{array}{c}\text { Roof } 3 \\
16\end{array}$ & $\begin{array}{c}\text { Roof } 4 \\
24\end{array}$ & $\begin{array}{c}\text { Roof } 5 \\
32\end{array}$ & $\begin{array}{c}\text { Roof } 6 \\
40\end{array}$ & $\begin{array}{c}\text { Roof } 7 \\
48\end{array}$ & $\begin{array}{c}\text { Floor } 1 \\
6\end{array}$ & $\begin{array}{c}\text { Floor } 2 \\
40\end{array}$ \\
\hline \multirow{3}{*}{2 VPP } & Trial 1 & 0.016646 & 0.019068 & 0.020394 & 0.021541 & 0.021947 & 0.021607 & 0.019876 & 0.003482 & 0.005745 \\
\hline & & 0.016746 & 0.019176 & 0.02051 & 0.021665 & 0.022077 & 0.021741 & 0.020006 & 0.003502 & 0.005778 \\
\hline & Trial 3 & 0.016561 & 0.018967 & 0.020286 & 0.021425 & 0.021836 & 0.021504 & 0.019796 & 0.003463 & 0.005717 \\
\hline \multirow{3}{*}{$1.5 \mathrm{VPP}$} & & 0.01281 & 0.014669 & 0.015709 & 0.01661 & 0.016904 & 0.016636 & 0.015315 & 0.002675 & 0.004427 \\
\hline & & 0.012526 & 0.014345 & 0.015367 & 0.016249 & 0.016546 & 0.016288 & 0.015002 & 0.002616 & 0.004333 \\
\hline & al 3 & 0.01264 & 0.014475 & 0.015509 & 0.016402 & 0.016697 & 0.016436 & 0.01514 & 0.002638 & 0.00437 \\
\hline \multirow{3}{*}{$1.0 \mathrm{VPP}$} & & 0.007602 & 0.008703 & 0.009345 & 0.009908 & 0.01006 & 0.009891 & 0.009103 & 0.001585 & 0.002639 \\
\hline & & 0.007638 & 0.008742 & 0.009387 & 0.009953 & 0.010104 & 0.009934 & 0.00914 & 0.001592 & 0.002646 \\
\hline & & 0.007633 & 0.008737 & 0.009379 & 0.009945 & 0.010101 & 0.009926 & 0.009136 & 0.00159 & 0.002641 \\
\hline \multirow{3}{*}{$0.5 \mathrm{VPP}$} & Trial 1 & 0.002444 & 0.002794 & 0.003007 & 0.0032 & 0.00323 & 0.00316 & 0.002902 & 0.000506 & 0.000842 \\
\hline & & 0.002455 & 0.002807 & 0.003019 & 0.003212 & 0.003246 & 0.003173 & 0.002914 & 0.00051 & 0.000841 \\
\hline & Trial 3 & 0.002392 & 0.002736 & 0.002945 & 0.003134 & 0.003163 & 0.003093 & 0.002841 & 0.000497 & 0.000824 \\
\hline
\end{tabular}




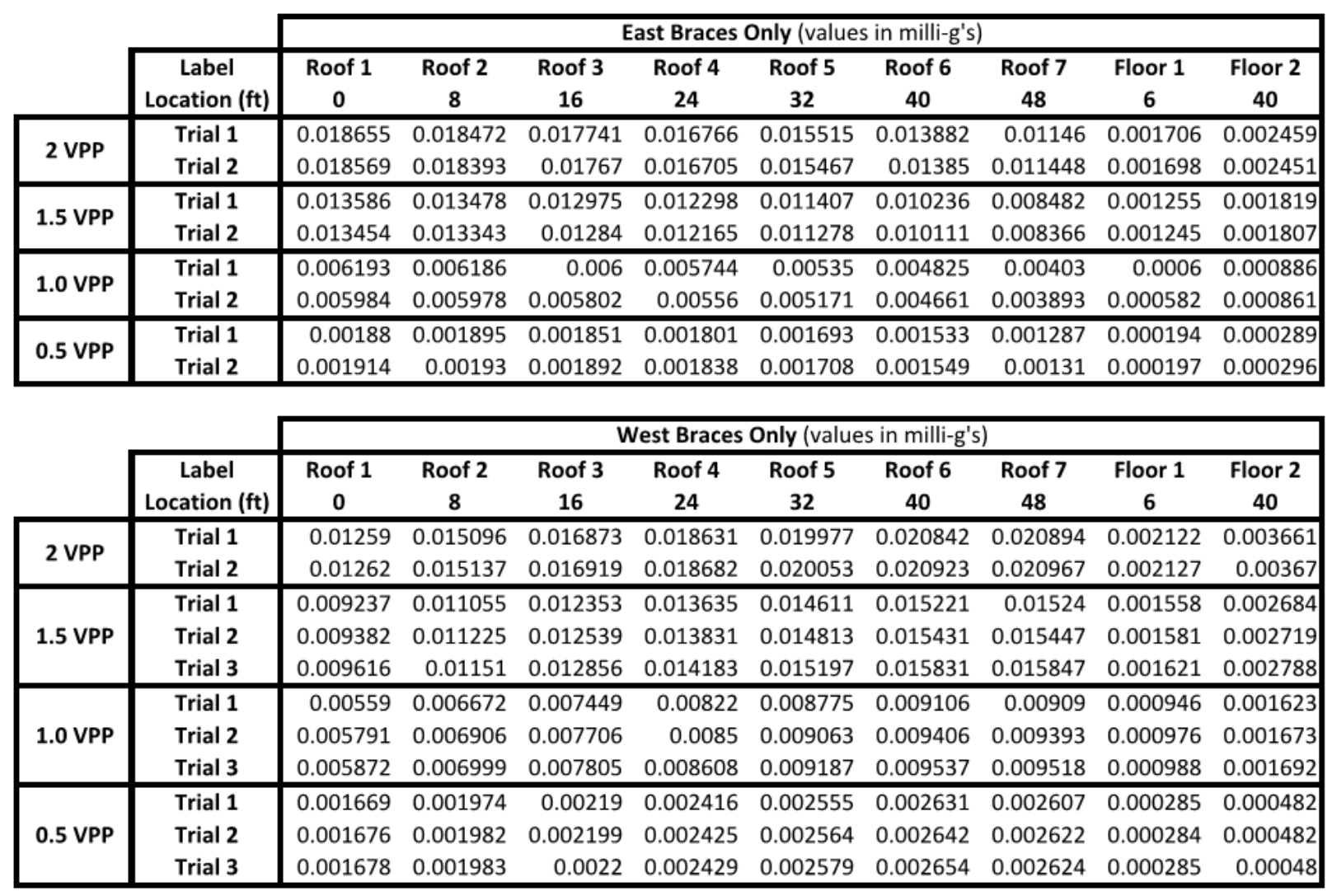

